\title{
Stabilization of Microbial Communities by Responsive Phenotypic Switching
}

\author{
Pierre A. Haas, ${ }^{1,2,3, *}$ Maria A. Gutierrez,${ }^{4}$ Nuno M. Oliveira, ${ }^{4,5, \dagger}$ and Raymond E. Goldstein ${ }^{4, \ddagger}$ \\ ${ }_{1}^{1}$ Max Planck Institute for the Physics of Complex Systems, Nöthnitzer Straße 38, 01187 Dresden, Germany \\ ${ }^{2}$ Max Planck Institute of Molecular Cell Biology and Genetics, Pfotenhauerstraße 108, 01307 Dresden, Germany \\ ${ }^{3}$ Center for Systems Biology Dresden, Pfotenhauerstraße 108, 01307 Dresden, Germany \\ ${ }^{4}$ Department of Applied Mathematics and Theoretical Physics, Centre for Mathematical Sciences, \\ University of Cambridge, Wilberforce Road, Cambridge CB3 OWA, United Kingdom \\ ${ }^{5}$ Department of Veterinary Medicine, University of Cambridge, Madingley Road, Cambridge CB3 OES, United Kingdom
}

(Dated: December 12, 2021)

\begin{abstract}
Clonal microbes can switch between different phenotypes and recent theoretical work has shown that stochastic switching between these subpopulations can stabilize microbial communities. This phenotypic switching need not be stochastic, however, but can also be in response to environmental factors, both biotic and abiotic. Here, motivated by the bacterial persistence phenotype, we explore the ecological effects of such responsive switching by analyzing phenotypic switching in response to competing species. We show how the stability of microbial communities with responsive switching differs generically from that of communities with stochastic switching only. To understand this effect, we go on to analyse simple two-species models. Combining exact results and numerical simulations, we extend the classical stability results for models of two competing species without phenotypic variation to the case where one of the two species switches, stochastically and responsively, between two phenotypes. In particular, we show that responsive switching can stabilize coexistence even when stochastic switching on its own does not affect the stability of the community.
\end{abstract}

\section{INTRODUCTION}

One of the classical results of theoretical ecology is that large random microbial communities are very likely to be unstable [1]. This is a statistical result, based on the analysis of random matrices representing the Jacobians of otherwise unspecified population dynamics [1]. It may appear to contradict Nature's large biodiversity, but actual biological interactions are of course not random: rather, they are the product of a long history of evolution, during which population dynamics pruned a possibly much larger set of species [2]. Nevertheless, the mathematical constraints from random matrix theory that restrict the evolution of this biodiversity can be revealed by this statistical take on population stability, because it enables some analysis of generic large ecological communities and their huge parameter space, and therefore complements the exact results for small systems that are not available for these larger systems. This has been demonstrated in a large body of work which, for example, analysed the effect of the interaction type and structure on the stability of the community [3-6], revealed the stabilizing effect of higher-order interactions and explicit resource dynamics [7, 8], or explored yet other related problems [9-13].

In this context, recent work has revealed that subpopulation structure such as phenotypic variation can stabilize microbial communities [14, 15]. In particular, we have argued that abundant phenotypic variation is generically destabilizing [15], essentially because introducing phenotypic variation increases the effective number of species in the system, which is known to be destabilizing [1,3]. More subtly however, stochastic switching to a rare phenotype such as the bacterial persister phenotype [16-18] can stabilize communities [15].

\footnotetext{
*haas@pks.mpg.de

$\dagger$ n.m.oliveira@damtp.cam.ac.uk

†.e.goldstein@damtp.cam.ac.uk
}

Although such stochastic phenotypic switching is optimal in infrequently changing environments [19], frequent environmental cues, be they biotic or abiotic, favor responsive phenotypic switching associated to some kind of sensing mechanism [19]. Such sensing is likely to be involved in switching to any phenotype resilient to stress conditions, akin to bacterial persisters [16-18]. Indeed, formation of persisters under stress has recently been associated with production of the "alarmone" ppGpp, suggesting a stress-dependent persistence response $[16,18]$. Data showing that sublethal antibiotic concentrations increase persister concentration [20] are also consistent with responsive contributions to the persistence switching rates. Earlier experiments exposing Escherichia coli to antibiotics suggested that direct sensing is absent from the persistence response [21], but these could not, as already noted in Ref. [19], exclude effects such as a dependence of switching rates on antibiotic concentration. The cues for this responsive switching may be toxins produced by other bacterial species, for example the colicinogens produced by certain strains of $E$. coli that have garnered attention in the context of bacterial "rock-paper-scissors" games [22-25].

Here, we address the ecological implications of this responsive switching by analyzing its effect on the stability of communities of competing microbes. In the first part of this paper, we therefore extend the two-phenotype model of Ref. [15] to include responsive phenotypic switching, and show how the statistical stability properties of this model differ from those of a model with stochastic switching only, even if the second phenotype is rare. These statistical results emphasize the importance of the type of phenotypic switching for stability, but leave unanswered the following question: under which conditions does phenotypic switching, be it stochastic or responsive, stabilize or destabilize microbial communities? We address this question in the second part of this paper: we extend the classical results for the stability of Lotka-Volterra systems [26] by analyzing a simple model of two competing species, in which one species switches, both stochastically 
and in response to the other species, between two phenotypes. Through numerical simulations and by deriving exact results for still simpler models, we show in particular that responsive switching promotes coexistence even in cases in which stochastic switching on its own does not affect the stability of the community.

\section{STATISTICAL STABILITY OF RESPONSIVE PHENOTYPIC SWITCHING}

In this Section, we introduce a model, which builds upon that of Ref. [15], for the competition of $N$ species that switch, both responsively and stochastically, between two phenotypes. We show that its statistical stability properties are generically different from those of the corresponding model with stochastic switching only.

\section{A. Model}

We consider the competition of $N \geqslant 2$ well-mixed species that have two phenotypes, $\mathrm{B}$ and $\mathrm{P}$, each, between which they switch stochastically. In addition, the B phenotypes of each species respond to the other species by switching to the corresponding $\mathrm{P}$ phenotype [Fig. 1(a)]. We denote by $B_{n}$ and $P_{n}$ the respective abundances of the $\mathrm{B}$ and $\mathrm{P}$ phenotypes of species $n$, for $1 \leqslant n \leqslant N$. With Lotka-Volterra competition terms [26], the dynamics of the vectors $\boldsymbol{B}=\left(B_{1}, B_{2}, \ldots, B_{N}\right)$ and $\boldsymbol{P}=\left(P_{1}, P_{2}, \ldots, P_{N}\right)$ are thus [27]

$$
\begin{aligned}
& \dot{\boldsymbol{B}}=\boldsymbol{B}(\boldsymbol{b}-\mathrm{C} \cdot \boldsymbol{B}-\varepsilon \mathrm{D} \cdot \boldsymbol{P})-\varepsilon \boldsymbol{B}(\boldsymbol{k}+\mathrm{R} \cdot \boldsymbol{B}+\mathrm{S} \cdot \boldsymbol{P})+\boldsymbol{\ell P}, \\
& \dot{\boldsymbol{P}}=\varepsilon \boldsymbol{P}(\boldsymbol{p}-\mathrm{E} \cdot \boldsymbol{B}-\mathrm{F} \cdot \boldsymbol{P})+\varepsilon \boldsymbol{B}(\boldsymbol{k}+\mathrm{R} \cdot \boldsymbol{B}+\mathrm{S} \cdot \boldsymbol{P})-\boldsymbol{\ell} \boldsymbol{P},
\end{aligned}
$$

where $\boldsymbol{b}, \boldsymbol{p}$ are growth rates, the nonnegative entries of the matrices C, D, E, F are competition strengths, $\boldsymbol{k}, \boldsymbol{\ell}$ are nonnegative rates of stochastic switching [28], and R, S are nonnegative rates of responsive switching. The diagonal entries of $R, S$ vanish, so that species do not switch phenotype in response to the presence of their own species. (a)

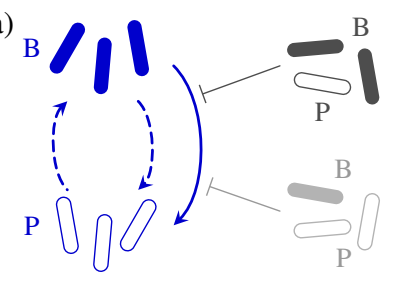

(b)

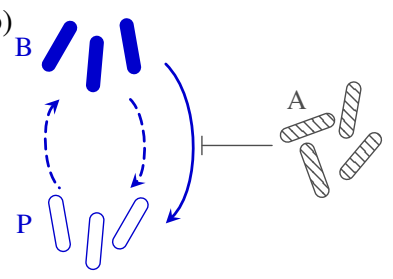

FIG. 1. Models of stochastic and responsive phenotypic switching. (a) In the model of Sec. II, each species has two phenotypes, B and $\mathrm{P}$, and switches stochastically between them. Moreover, the B phenotype of each species responds to other species by switching to the P phenotype. (b) In the two-species model of Sec. III, the second species has a single competitor phenotype A, which causes B to switch to P. Dashed lines: stochastic switching. Solid lines: responsive switching.
The functional form of the responsive switching rates in Eqs. (1), with a direct dependence on the competitor abundances, implies a neglect of the toxin dynamics that are the chemical cues of the reponsive switching. This is justified since our model aims to elucidate the effect of this responsive switching on stability, rather than that of toxin dynamics [29]; the same argument justifies, e.g., neglecting resource dynamics in spite of their known effect on stability [8]. The linear dependence of responsive switching on the abundances of other species is, however, a simplifying assumption, just as the logistic Lotka-Volterra competition terms [26] in Eqs. (1) are the simplest choice of competition terms. While a quantitative study of the dynamics of phenotypic switching in competing microbial populations may therefore require more realistic functional forms of the competition terms and switching rates, we stress that these would reduce to those in Eqs. (1) close to equilibrium. Moreover, as shown in Appendix A, the logistic nonlinearities in Eqs. (1) are sufficient for the dynamics to be bounded and hence realistic biologically. For these reasons, we believe these simplifying assumptions to be appropriate for the qualitative analysis of phenotypic dynamics and in particular of the stability of the equilibria of Eqs. (1) in this paper.

The parameter $\varepsilon$ in Eqs. (1) scales the competition strengths of the $\mathrm{P}$ phenotypes, and the stochastic and responsive switching rates into the $\mathrm{P}$ phenotypes. In the limit $\varepsilon \ll 1$, the $\mathrm{P}$ phenotype is therefore a slowly growing and weakly competing phenotype such as bacterial persisters [16-18].

\section{B. Reduced and Averaged Models}

How do phenotypic variation and switching affect the stability of a microbial community? This is the biological question that we will have in mind throughout this paper.

To answer this question, we can distinguish between analyzing (a) the effect of phenotypic variation and switching and (b) the effect of the type of phenotypic switching. In this context, it is tempting to compare the mathematical stability of equilibria of Eqs. (1) to that of equilibria of corresponding reduced models: (i) a reduced model without phenotypic variation in which $\boldsymbol{P}=\mathbf{0}, \boldsymbol{k}=\boldsymbol{\ell}=\mathbf{0}, \mathrm{R}=\mathrm{S}=\mathrm{O}$, and (ii) a reduced model with stochastic switching only in which $R=S=O$. Such a comparison does not however answer our biological question, because the populations at equilibrium of Eqs. (1) and the corresponding reduced models are in general different, and a direct comparison cannot therefore decide whether stability differences result from this difference of populations, or from phenotypic variation and switching.

To understand the effect of phenotypic variation and switching, we do not therefore compare Eqs. (1) to these reduced models, but instead to averaged models that have the same population at equilibrium as Eqs. (1). While reduced models take values of competition parameters or switching rates from Eqs. (1), the averaged models replace these values with effective values that are determined by the condition of equality of populations at equilibrium. We introduced such averaging of models in Ref. [15]. There, we pointed out that equality of populations at equilibrium does not lead to simple relations between the eigenvalues of the corresponding Jacobians, so 
there is no reason to expect their stability properties to be the same. The present discussion develops these ideas.

While stability differences between Eqs. (1) and the corresponding averaged models thus reveal the biological role of phenotypic variation and switching, stability differences between the reduced and averaged models stem from the differences of the reduced and averaged model parameters. In an actual biological community, these parameters can evolve independently from the evolution of phenotypic substructure, and so stability differences need not result from the phenotypic substructure. This raises interesting questions akin to those asked in Ref. [19]: For example, does evolving a more complex phenotypic substructure require more evolutionary adaptations than evolving the effective parameters directly? Answering such questions requires, however, coupling Eqs. (1) to an evolutionary model. This is beyond the scope of this paper, in which we will therefore focus on the stability differences between Eqs. (1) and the corresponding averaged models, which can be imputed directly to phenotypic variation and switching.

The above discussion of reduced and averaged models has remained rather abstract. We now apply these ideas to Eqs. (1) by introducing the averaged models explicitly.

The averaged model with stochastic only is the model that we have analyzed in Ref. [15],

$$
\begin{aligned}
\dot{\boldsymbol{B}} & =\boldsymbol{B}(\boldsymbol{b}-\mathrm{C} \cdot \boldsymbol{B}-\varepsilon \mathrm{D} \cdot \boldsymbol{P})-\varepsilon \boldsymbol{k}^{\prime} \boldsymbol{B}+\boldsymbol{\ell P}, \\
\dot{\boldsymbol{P}} & =\varepsilon \boldsymbol{P}(\boldsymbol{p}-\mathrm{E} \cdot \boldsymbol{B}-\mathrm{F} \cdot \boldsymbol{P})+\varepsilon \boldsymbol{k}^{\prime} \boldsymbol{B}-\boldsymbol{\ell P} .
\end{aligned}
$$

To establish the correspondence between Eqs. (1) and Eqs. (2), we notice that an equilibrium $\mathcal{E}=\left(\boldsymbol{B}_{*}, \boldsymbol{P}_{*}\right)$ of Eqs. (1) is also an equilibrium of Eqs. (2) if $\boldsymbol{k}^{\prime}=\boldsymbol{k}+\mathrm{R} \cdot \boldsymbol{B}_{*}+\mathrm{S} \cdot \boldsymbol{P}_{*}$ and all other growth rates, competition strengths, and switching rates are unchanged. While we shall see below that, as expected, these models do not have identical stability properties, this shows that, at equilibrium, responsive switching only modifies the effective rate of stochastic switching. At equilibrium, it is not therefore possible to distinguish between purely stochastic switching and a dependence of switching rates on the presence of other species. This aspect, we have noted in the Introduction, has previously been discussed from an experimental point of view in the context of $E$. coli persistence [19,21]. Conversely, in a reduced model, the rate $\boldsymbol{k}$ of stochastic switching would remain unchanged from Eqs. (1); stability differences between Eqs. (2) and this reduced model could therefore also result, independently from responsive switching, from evolution of the rate of stochastic switching.

For reference in the discussion of simplified models in the next section, we note that we can also associate to Eqs. (1) an averaged model without phenotypic variation,

$$
\dot{\boldsymbol{B}}^{\prime}=\boldsymbol{B}^{\prime}\left(\boldsymbol{b}^{\prime}-\mathrm{C}^{\prime} \cdot \boldsymbol{B}^{\prime}\right),
$$

with a coexistence equilibrium $\boldsymbol{B}_{*}^{\prime}=\mathrm{C}^{-1} \cdot \boldsymbol{b}^{\prime}$. As we have noted in Ref. [15], this equilibrium is consistent with an equilibrium $\mathcal{E}=\left(\boldsymbol{B}_{*}, \boldsymbol{P}_{*}\right)$ of Eqs. (1) or (2) if and only if the population sizes, births, and competition at equilibrium are equal, i.e.

$$
\begin{aligned}
\boldsymbol{B}_{*}^{\prime} & =\boldsymbol{B}_{*}+\boldsymbol{P}_{*}, \quad \boldsymbol{b}^{\prime} \boldsymbol{B}_{*}^{\prime}=\boldsymbol{b} \boldsymbol{B}_{*}+\varepsilon \boldsymbol{p} \boldsymbol{P}_{*} \\
\boldsymbol{B}_{*}^{\prime} \mathrm{C}^{\prime} \boldsymbol{B}_{*}^{\prime} & =\boldsymbol{B}_{*} \mathrm{C} \boldsymbol{B}_{*}+\varepsilon\left(\boldsymbol{B}_{*} \mathrm{D} \boldsymbol{P}_{*}+\boldsymbol{P}_{*} \mathrm{E} \boldsymbol{B}_{*}+\boldsymbol{P}_{*} \mathrm{~F} \boldsymbol{B}_{*}\right) .
\end{aligned}
$$

These conditions uniquely determine the effective parameters $\boldsymbol{b}^{\prime}$ and $\mathrm{C}^{\prime}$ of the averaged model and its equilibrium $\boldsymbol{B}_{*}^{\prime}$. Again, the corresponding reduced model would inherit its birth rates $\boldsymbol{b}$ and competition parameters C from Eqs. (1). Stability differences between this reduced model and Eqs. (3) need not therefore result from the phenotypic substructure, but could also stem from evolution of the B phenotypes.

We emphasize that this averaging does not imply that the dynamics of the sum $\boldsymbol{B}+\boldsymbol{P}$ resulting from Eqs. (1) are of the averaged form (3). In the same way, the dynamics of Eqs. (2) are different, away from equilibrium, from those of Eqs. (1). It is for this reason that correspondence at equilibrium does not, as we have already noted above, translate to corresponding stability properties. In what follows, we therefore analyze these stability properties to understand the effect of phenotypic variation and switching on stability.

\section{Results}

We begin by comparing the stability of Eqs. (1) and Eqs. (2). In the spirit of the random matrix approach to statistical stability, we sample the model parameters of Eqs. (1) randomly, and compute stability statistics. Since the coexistence equilibria of Eqs. (1) cannot be found in closed form, we cannot directly sample the model parameters; rather, as discussed in more detail in Appendix A, we sample the coexistence state itself and some model parameters directly, leaving linear equations to be solved for the remaining parameters to ensure that the chosen coexistence state is steady [15].

For these random systems, we find that, as the number $N$ of species increases, stable coexistence states of Eqs. (1) are increasingly unlikely to be stable with the dynamics of Eqs. (2), and vice versa: stable coexistence with responsive switching is increasingly unlikely to be stable with stochastic switching only, and vice versa [Fig. 2(a)]. This trend persists for $\varepsilon \ll 1$, although the probabilities are reduced in magnitude [Fig. 2(b)]. Responsive switching can thus stabilize and destabilize equilibria of Eqs. (1). However, coexistence is less likely to be stable with responsive switching than with stochastic switching only [Fig. 2(c)], although the probabilities are nearly equal for small $\varepsilon$ [Fig. 2(c), inset].

In the limit $\varepsilon \ll 1$, coexistence states can be determined in closed form by asymptotic expansion in the small parameter $\varepsilon$. Writing $\boldsymbol{B}_{*}=\boldsymbol{B}_{\mathbf{0}}+\varepsilon \boldsymbol{B}_{\mathbf{1}}+O\left(\varepsilon^{2}\right), \boldsymbol{P}_{*}=\boldsymbol{P}_{\mathbf{0}}+\varepsilon \boldsymbol{P}_{\mathbf{1}}+O\left(\varepsilon^{2}\right)$, we find $\boldsymbol{B}_{\mathbf{0}}=\mathrm{C}^{-1} \cdot \boldsymbol{b}, \boldsymbol{P}_{\mathbf{1}}=\left(\boldsymbol{k}+\mathrm{R} \cdot \boldsymbol{B}_{\mathbf{0}}\right) \boldsymbol{B}_{\mathbf{0}} / \boldsymbol{\lambda}$, but $\boldsymbol{B}_{\mathbf{1}}=\boldsymbol{P}_{\mathbf{0}}=\mathbf{0}$, as derived in more detail in Appendix A. We thence obtain asymptotic expressions for the Jacobians (Appendix A), similarly to calculations in Ref. [15]. Sampling asymptotic coexistence equilibria using this solution, we confirm our previous findings [Fig. 2(b)], although results differ slightly at a quantitative level because of the different sampling methods.

The models with and without responsive switching thus generically lead to different stability results. The Jacobians at $\mathcal{E}, J_{*}$ with responsive switching and $\mathrm{K}_{*}$ with stochastic switching only, are related by

$$
J_{*}=\mathrm{K}_{*}+\varepsilon\left(\begin{array}{c|c}
-\boldsymbol{B}_{*} \mathrm{R} & -\boldsymbol{B}_{*} \mathrm{~S} \\
\hline \boldsymbol{B}_{*} \mathrm{R} & \boldsymbol{B}_{*} \mathrm{~S}
\end{array}\right),
$$



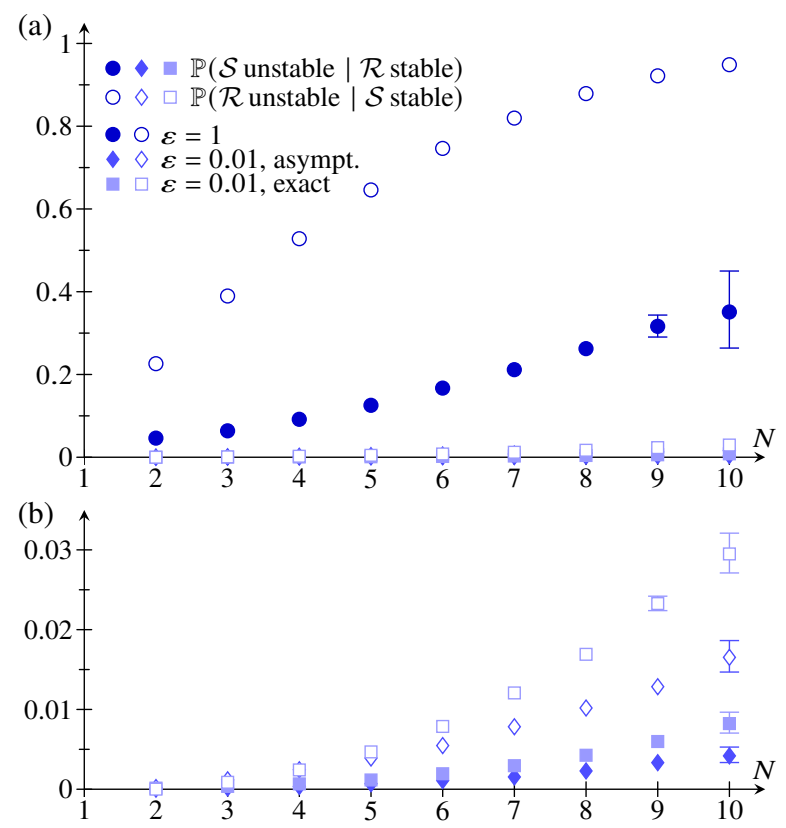

(c) ${ }_{\uparrow} p=\mathbb{P}(\mathcal{R}$ stable $) / \mathbb{P}(\mathcal{S}$ stable $)$

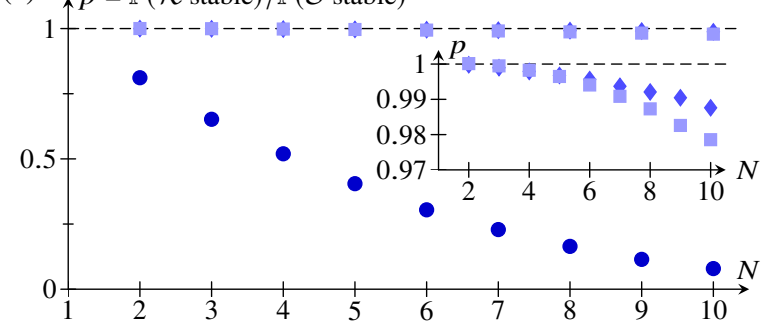

FIG. 2. Stability of random microbial communities with responsive phenotypic switching. (a) Probability of a random equilibrium that is stable in the model with responsive switching $\mathcal{R}$ [Eqs. (1)] or in the model with stochastic switching only $\mathcal{S}$ [Eqs. (2)] being unstable in the other model, as a function of the number of species in the system, $N$. (b) Same plot, but focused on low probabilities. (c) Ratio of the probabilities of random equilibria of $\mathcal{R}$ and $\mathcal{S}$ being stable. Inset: same plot, focused on small probability differences. Probabilities were estimated from up to $5 \cdot 10^{8}$ random systems each. Parameter values: $\varepsilon=1$ and $\varepsilon=0.01$; for the latter value, both exact and asymptotic equilibria were computed. Error bars are $95 \%$ confidence intervals [30] larger than the plot markers.

which follows from the calculations in Appendix A. We stress that this is an exact result and not an asymptotic approximation. Even in view of this linear relation, the fact that the two models give different stability results is not fundamentally surprising [15]: this simply reflects the fact that linear relations between matrices do not imply linear relations between their determinants. What is perhaps more surprising is that, although $\mathrm{J}_{*}=\mathrm{K}_{*}+O(\varepsilon)$, the stability results differ even in the limit $\varepsilon \ll 1$. We have previously related behavior of this ilk to the possibility of eigenvalues with small real parts being stabilized or destabilized by higher-order terms in the expansion [15].

We had to restrict to small values of $N$ when computing the numerical results in Fig. 2, because it becomes increasingly difficult to sample increasingly rare [1,3] stable systems as $N$ increases. The strength of the classical random matrix ap- proach $[1,3]$ to stability is of course that it can often circumvent this difficulty by analyzing random Jacobians without further specification of the underlying population dynamics and hence of their equilibria. This simplification was not however available for the questions addressed here, because we needed to relate "full" and averaged models (in this case, with and without responsive switching). Nonetheless, the trends in Fig. 2 suggest that the stability differences found numerically there are amplified for larger values of $N$.

There is, however, a more important limitation of this stability analysis: one may argue that instability of an equilibrium is not biologically significant, because it does not imply extinction of a species, since the dynamics, perturbed away from the unstable equilibrium, may converge to a different equilibrium, a limit cycle, or a more complex attractor, and so the species may still coexist. A more relevant question biologically is therefore: does stability of one model (with responsive switching or with stochastic switching only) not only fail to predict stability, but also fail to predict coexistence in the other model? To address this question, we evolve unstable equilibria numerically and, as described in Appendix A, determine whether the species coexist permanently [31].
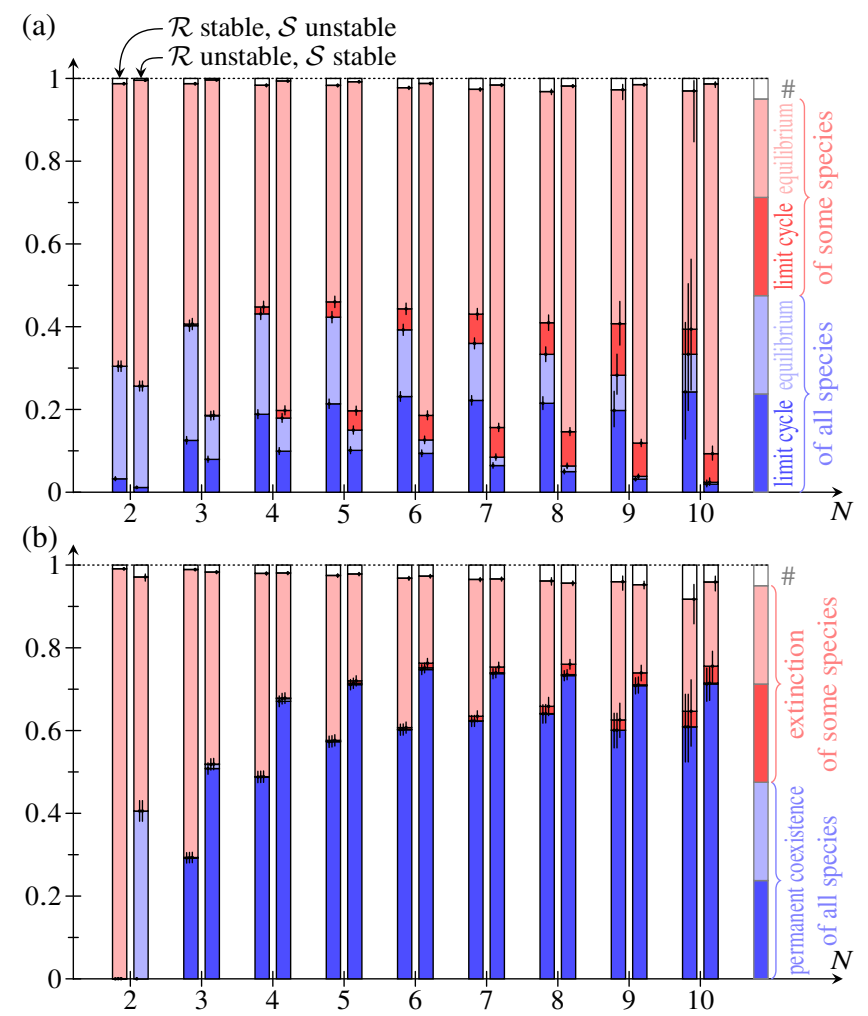

FIG. 3. Distributions, in the models with responsive switching $\mathcal{R}$ [Eqs. (1)] or with stochastic switching only $\mathcal{S}$ [Eqs. (2)], of the long-time dynamics of unstable equilibria that are stable in the other model. Distributions are shown for exact equilibria with (a) $\varepsilon=1$, (b) $\varepsilon=0.01$, and different numbers of species $N$. Each distribution was estimated by numerical integration of up to $5 \cdot 10^{3}$ unstable systems. For a small proportion of the systems (\#), the numerical solution did not converge. Vertical bars represent $95 \%$ confidence intervals [30]. 
Figure 3 shows the distributions of the long-time dynamics of equilibria that are unstable with responsive switching or with stochastic switching only, respectively, but are stable in the other model. The possible long-time dynamics are permanent coexistence or extinction of some species; in both cases, we distinguish between convergence of the remaining species to an equilibrium or to a limit cycle. The small proportion of systems for which the numerical solution does not converge (Fig. 3) may include systems in which more complex attractors arise. We note in particular that extinction of some species is not a rare outcome (Fig. 3): it is actually the most likely outcome if both phenotypes have similar abundances [ $\varepsilon=1$, Fig. 3(a)], while convergence to a limit cycle of all species is more likely if one phenotype is rare [ $\varepsilon \ll 1$, Fig. 3(b)].

From these distributions, we estimate, for both models, the probabilities of extinction of some species for systems perturbed away from an equilibrium that is stable in the other model (Fig. 4). The increase of these probabilities with the number of species $N$ qualitatively matches the increase of the probabilities of an equilibrium that is stable in one model being unstable in the other model. We can therefore extend our previous conclusion to a more biologically relevant one: stability of an equilibrium in one model does not even in general
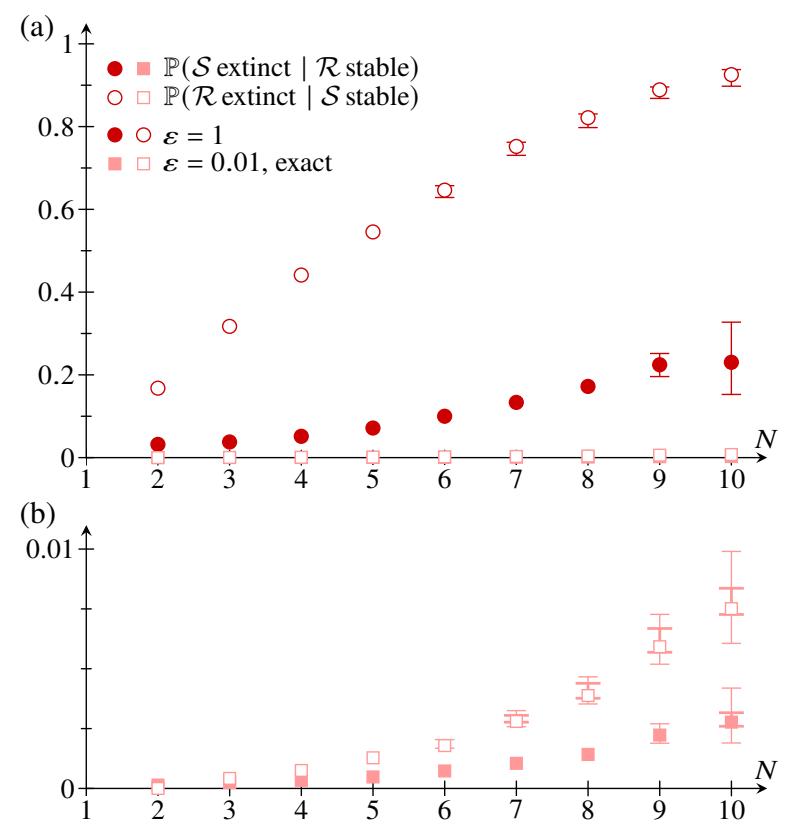

FIG. 4. Permanent coexistence in random microbial communities with responsive phenotypic switching. (a) Probability, in the models with responsive switching $\mathcal{R}$ [Eqs. (1)] and with stochastic switching only $\mathcal{S}$ [Eqs. (2)], of extinction of some species of a random system perturbed away from an equilibrium that is stable in the other model, as a function of the number of species in the system, $N$. (b) Same plot, but focused on low probabilities. Exact equilibria were obtained for $\varepsilon=1$ and $\varepsilon=0.01$. Each probability was computed from up to $5 \cdot 10^{8}$ random systems and up to $5 \cdot 10^{3}$ random systems having an equilibrium with different stability in models $\mathcal{R}$ and $\mathcal{S}$. Thick error bars correct the estimated probabilities for the systems in which the numerical solution of the long-time behavior did not converge (Fig. 3); thin error bars add $95 \%$ confidence intervals [30]. Only those error bars larger than the plot markers are shown. imply permanent coexistence in the other model.

Finally, we could similarly compare the stability of Eqs. (1) and Eqs. (3). However, in view of the correspondence between Eqs. (1) and Eqs. (2), the qualitative conclusions of such a comparison must parallel those of comparing models with stochastic switching only and averaged models without phenotypic variation. These two models we compared in Ref. [15], where we concluded that stochastic switching to an abundant phenotype is destabilizing, but stochastic switching to a rare phenotype, corresponding to $\varepsilon \ll 1$, is stabilizing. Here, we can therefore conclude similarly that responsive switching to an abundant phenotype generically destabilizes the community (compared to the case in which there is no phenotypic variation), but that responsive switching to a rare phenotype has a stabilizing effect.

However, all of these results are fundamentally statistical in nature. While they show how responsive phenotypic switching affects stability on average, they do not yield any insight into the conditions under which phenotypic switching stabilizes or destabilizes the community. To understand the mechanisms underlying these stability differences, we therefore complement this statistical analysis with an analysis of reduced twospecies models in the next Section.

\section{STABILIZATION OF COEXISTENCE BY RESPONSIVE SWITCHING}

In this Section, we reduce the $N$-species model (1) to a two-species model. In the context of this model, we analyze the mechanisms by which stochastic and responsive switching affect stability numerically, and, on further simplification of the model, we obtain exact results extending the classical results [26], which we rederive in Appendix B, for the stability of the two-species Lotka-Volterra competition model.

\section{A. Two-species models}

We consider the competition between two well-mixed species: the first species has a single phenotype, A, while the second one has two phenotypes, $\mathrm{B}$ and $\mathrm{P}$, between which it switches stochastically. Moreover, phenotype B responds to the competitor phenotype A by switching to P [Fig. 1(b)]. To interpret this model in terms of actual biological systems, it is useful to think of $\mathrm{P}$ as a phenotype resilient to stress conditions, akin to the bacterial persister phenotype [16-18], and to which the common, "normal" phenotype B switches in response to toxins produced by A. With this interpretation and for simplicity, we shall sometimes refer to B, P, A and their respective abundances $B, P, A$ as bacteria, persisters, and competitors [32], and we shall sometimes assume the model parameters to scale accordingly.

Thus, again using Lotka-Volterra competition terms [26], the nondimensionalized dynamics of the system are

$$
\begin{aligned}
& \dot{B}=B(1-\alpha A-B-\kappa P)-\beta A B-\gamma B+\delta P, \\
& \dot{P}=P(\mu-\xi A-\varpi B-\varsigma P)+\beta A B+\gamma B-\delta P, \\
& \dot{A}=A(\zeta-\eta A-\vartheta B-\iota P),
\end{aligned}
$$


wherein $\alpha, \beta, \gamma, \delta, \zeta, \eta, \vartheta, \iota, \kappa, \mu, \xi, \varpi, \varsigma \geqslant 0$ are dimensionless nonnegative parameters. To obtain this form of the equations, we have scaled time and the population sizes so as to remove the parameters that would otherwise appear in the logistic growth term $B(1-B)$ of the bacteria in the absence of persisters and competitors [33]. The interpretation of the model parameters in Eqs. (6) is given in Table I.

The full two-species model (6) does not easily lend itself to analytical progress. Indeed, on computing a Gröbner basis [34] for the steady-state version of Eqs. (6) using MAтHEMATICA (Wolfram, Inc.), we find that even just computing the coexistence equilibria of Eqs. (6) requires solving a quartic equation. To enable some analytical progress, we therefore introduce a simplified version of Eqs. (6),

$$
\begin{aligned}
& \dot{B}=B(1-\alpha A-B)-\beta A B-\gamma B+\delta P, \\
& \dot{P}=\beta A B+\gamma B-\delta P, \\
& \dot{A}=A(\zeta-\eta A-\vartheta B-\iota P) .
\end{aligned}
$$

Compared to the full system (6), all but one of the competition terms involving the persisters have been removed in this system. We stress that Eqs. (7) are not the asymptotic limit of Eqs. (6) for slowly growing and weakly competing persisters. However, it will turn out that including this one term, while leaving the system amenable to analytical progress, introduces nontrivial behaviour.

We will also consider two other simplified models that are obtained similarly:

$$
\begin{aligned}
& \dot{B}=B(1-\alpha A-B)-\beta A B-\gamma B+\delta P, \\
& \dot{P}=\mu P+\beta A B+\gamma B-\delta P, \\
& \dot{A}=A(\zeta-\eta A-\vartheta B),
\end{aligned}
$$

\begin{tabular}{|c|c|c|}
\hline group & parameter $^{\mathrm{a}}$ & interpretation $^{\mathrm{b}}$ \\
\hline \multirow{4}{*}{ 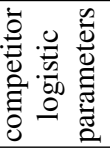 } & $\alpha$ & magnitude of effect of B-A interaction on B \\
\hline & $\zeta$ & growth rate of $\mathrm{A}$ \\
\hline & $\eta$ & strength of within-species competition of A \\
\hline & $\vartheta$ & magnitude of effect of B-A interaction on A \\
\hline \multirow{3}{*}{ 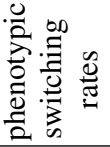 } & $\beta$ & rate of responsive switching from $\mathrm{B}$ to $\mathrm{P}$ \\
\hline & $\gamma$ & rate of stochastic switching from $\mathrm{B}$ to $\mathrm{P}$ \\
\hline & $\delta$ & rate of stochastic switching from $\mathrm{P}$ to $\mathrm{B}$ \\
\hline \multirow{6}{*}{ 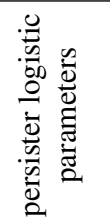 } & $\iota$ & magnitude of effect of P-A interaction on $\mathrm{A}$ \\
\hline & $\kappa$ & magnitude of effect of B-P interaction on B \\
\hline & $\mu$ & growth rate of $\mathrm{P}$ \\
\hline & $\xi$ & magnitude of effect of P-A interaction on P \\
\hline & $\varpi$ & magnitude of effect of P-B interaction on $\mathrm{P}$ \\
\hline & $\varsigma$ & strength of within-species competition of $\mathrm{P}$ \\
\hline
\end{tabular}

TABLE I. Parameters of the two-species model (6), representing the interactions in Fig. 1(b), and their interpretations. The parameters have been divided into three groups. Only one of the persister logistic parameters appears in each of the simplified models (7), (8), (9).

\footnotetext{
a The equations have been nondimensionalized by rescaling time and population abundances to remove two (dimensional) parameters, viz. the growth rate and the strength of the within-species competition of $\mathrm{B}$.

${ }^{\mathrm{b}}$ Phenotype abbreviations (B: bacteria; P: persisters; A: competitors) used in this column are as in Fig. 1(b).
}

and

$$
\begin{aligned}
& \dot{B}=B(1-\alpha A-B-\kappa P)-\beta A B-\gamma B+\delta P, \\
& \dot{P}=\beta A B+\gamma B-\delta P, \\
& \dot{A}=A(\zeta-\eta A-\vartheta B) .
\end{aligned}
$$

The three simplified models (7), (8), (9) thus correspond to allowing exactly one of $\iota, \mu, \kappa$ to be nonzero. The equilibria of the analogous simplified models corresponding to the remaining logistic parameters involving persisters, viz. $\xi, \varpi, \varsigma$, are determined by equations that are at least cubic, and hence do not allow much analytical progress. While we will not therefore analyze these three remaining models in detail, we will briefly discuss them when analyzing the full model (6) numerically.

We have not explicitly introduced a positive parameter $\varepsilon \lesssim 1$ scaling the competition dynamics of and switching rates to persisters (to keep the expressions that arise in the subsequent analytical calculations simple), but we expect

$$
\alpha, \delta, \zeta, \eta, \vartheta=O(1), \quad \beta, \gamma, \iota, \kappa, \mu, \xi, \varpi, \varsigma=O(\varepsilon)
$$

from the interpretation of the model in terms of bacteria, persisters, and competitors and by comparison of Eqs. (1) and (6). We will not assume $\varepsilon \ll 1$ in our analysis, but, when discussing the simplified models (7), (8), (9) below, we will sometimes invoke $\varepsilon \lesssim 1$ to impose additional inequalities relating the parameters.

\section{B. Simplified two-species models: stability diagrams}

In this subsection, we obtain analytical results that describe the feasibility and stability of the coexistence equilibria of the simplified models (7), (8), (9). We will find that responsive switching can stabilize coexistence even though stochastic switching does not affect stability (in the averaged sense discussed in Section II and Appendix B).

\section{Analysis of the simplified model (7)}

The simplified model (7) has five steady states: three trivial steady states,

$$
\mathcal{O}=(0,0,0), \quad \mathcal{A}=(0,0, \zeta / \eta), \quad \mathcal{B}=(1, \gamma / \delta, 0),
$$

and, if $\beta \iota \neq 0$, two coexistence equilibria,

$$
\mathcal{C}_{+}=\left(b_{+}, p_{+}, a_{+}\right), \quad \mathcal{C}_{-}=\left(b_{-}, p_{-}, a_{-}\right) .
$$

If $\beta \iota=0$, there is but a single coexistence state, $\mathcal{C}=(b, p, a)$. The complicated expressions for the coordinates of the coexistence states $\mathcal{C}_{+}, \mathcal{C}_{-}$and $\mathcal{C}$ are given in Appendix $\mathrm{C}$, to which we also postpone the detailed calculations determining the feasibility and stability of these equilibria. Here, we summarize the results and discuss the stability of coexistence.

First, if $\beta=0$, i.e. in the absence of responsive switching, the calculations in Appendix $\mathrm{C}$ show that $\mathcal{C}$ is stable if and only if $\vartheta+\gamma \iota / \delta<\zeta<\eta / \alpha$ (Fig. 5). We now ask: how does stable 

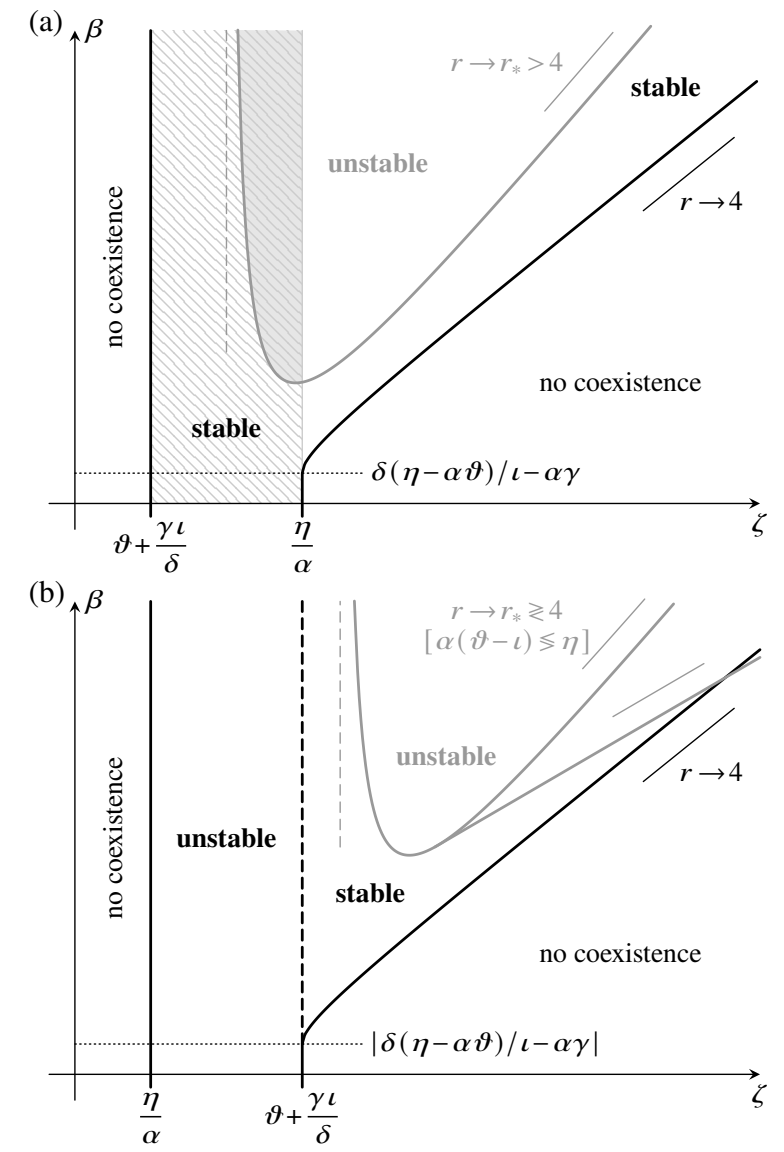

FIG. 5. Feasibility and stability of the coexistence states $\mathcal{C}_{ \pm}$of the simplified model (7) in the $(\zeta, \beta)$ diagram for $\iota \neq 0$, in the cases (a) $\eta / \alpha>\vartheta+\gamma \iota / \delta$ and (b) $\eta / \alpha<\vartheta+\gamma \iota / \delta$, assuming scalings (10). Coexistence is feasible in the region bounded by the thick solid black lines. Both $\mathcal{C}_{+}$and $\mathcal{C}_{-}$are unstable in the region marked "unstable", but $\mathcal{C}_{-}$is stable (and $\mathcal{C}_{+}$is unstable) in the region marked "stable", as discussed in the text and Appendix C. The exact boundary of the region of instability that arises at sufficiently large $\beta$ (grey lines) must be computed numerically; a region of stability at large $\zeta, \beta$ must exist in case (a), but only exists in case (b) if $\alpha(\vartheta-\iota)<\eta$. The boundary of this region asymptotes to the straight line $\beta \iota / \alpha \delta \zeta \equiv r=r_{*}$, where $r_{*}$ depends only on $\alpha(\vartheta-\iota) / \eta$ (Appendix C). In the hatched region of parameter space, the trivial steady states $\mathcal{A}$ and $\mathcal{B}$ are unstable, and the two species coexist permanently (Appendix $\mathrm{C}$ ). This is also the region of stable steady-state coexistence for averaged models of Eqs. (7) without phenotypic variation and with stochastic switching only (Appendix B). In the shaded region of parameter space (which need not exist), all steady states of Eqs. (7) are unstable.

coexistence in this case relate to stable coexistence in the classical two-species Lotka-Volterra model without phenotypic variation [26]? As discussed in Sec. II, answering this question requires establishing a correspondence between Eqs. (7) and a model without phenotypic variation. In Appendix B, we show how such a model arises as an average of Eqs. (7), and analyze its equilibria. In particular, we find that there is a one-to-one correspondence, both in terms of parameters and in terms of feasibility and stability, between Eqs. (7) and this averaged model without phenotypic variation. In other words, stochastic switching does not affect stability.
Next, if $\iota=0$, i.e. without persister-competitor interactions, $\mathcal{C}$ is stable if and only if $\vartheta<\zeta<\eta / \alpha$ for sufficiently small $\beta$. Coexistence is unstable at large $\beta$, but the limit $\iota \rightarrow 0$ is singular (Appendix C). The behavior that we report below for $\beta \iota \neq 0$ thus requires both responsive switching and persistercompetitor interactions.

If $\beta \iota \neq 0$, an additional region of feasibility arises, compared to the case $\beta \iota=0$, for $\zeta>\max \{\vartheta+\gamma \iota / \delta, \eta / \alpha\}$, i.e. for sufficiently fast competitor growth, and at sufficiently large $\beta$, say $\beta>\beta_{*}$ (Fig. 5). The minimal value $\beta_{*}$ can be determined in terms of the other model parameters in closed form (Appendix C). In particular,

$$
\beta_{*}>\beta_{*}^{\min }=\left|\frac{\delta}{\iota}(\eta-\alpha \vartheta)-\alpha \gamma\right| .
$$

With reference to the persister scalings (10), it is important to note that $\beta_{*}^{\min }=O(\varepsilon)$ is possible even if $\iota=O(\varepsilon)$, provided that $\eta-\alpha \vartheta \lesssim O\left(\varepsilon^{2}\right)$, i.e. provided that the intra-species competitions of phenotypes B and A are sufficiently close to their inter-species competitions.

The stability boundaries at $\beta \iota \neq 0$ are more intricate. One of the coexistence states $\mathcal{C}_{ \pm}$is always unstable, but the other may be stable (Appendix $C$ ). In the discussion that follows, we shall therefore focus on the second state, and also assume that $\iota<\vartheta$ and $\iota<\delta$; this is consistent with the expected scalings (10). There is then a region of parameter space for large $\beta$ in which coexistence is unstable (Fig. 5). The boundary of this region must be determined numerically, but asymptotic calculations (Appendix C) show that coexistence is stable for all $\beta$ if $\zeta-\vartheta-\gamma \iota / \delta>0$ is small enough. This region of stability extends to large $\beta$ and $\zeta$ if and only if $\alpha(\vartheta-\iota)>\eta$ (Fig. 5). Numerical examples do suggest that there is a single (unbounded) region of instability as sketched in Fig. 5, but we do not have a proof of this.

To understand the effect of responsive switching on the stability of the community, we compare the stability diagram thus obtained to that of the corresponding averaged model without phenotypic variation. Appendix B shows that this comparison also compares the stability of the equilibria of the model with responsive switching and those of an averaged model with stochastic switching only. Coexistence is stable in the averaged models precisely if $\vartheta+\gamma \iota / \delta<\zeta<\eta / \alpha$ (Fig. 5), as proved in Appendix B. Hence, because of the competition between phenotypes $\mathrm{P}$ and $\mathrm{A}$ for $\iota \neq 0$, sufficiently large levels of responsive switching are stabilizing in $\zeta>\max \{\vartheta+\gamma \iota / \delta, \eta / \alpha\}$ (Fig. 5), i.e. for fast-growing competitors.

The steady states $\mathcal{A}$ and $\mathcal{B}$, in which one species is extinct, are stable if and only if $\zeta>\eta / \alpha$ and $\zeta<\vartheta+\gamma \iota / \delta$, respectively (Appendix C). Their stability is thus independent of $\beta$. Responsive switching does not therefore help in driving the competitors to extinction, but the above shows that it makes stable steady-state coexistence possible in $\zeta>\max \{\vartheta+\gamma \iota / \delta, \eta / \alpha\}$ where extinction of bacteria and persisters is the only possible steady state at $\beta=0$.

However, if $\eta / \alpha>\vartheta+\gamma \iota / \delta$, the region of instability at large $\beta$ may intersect [Fig. 5(a)] the range $\vartheta+\gamma \iota / \delta<\zeta<\eta / \alpha$ in which, as discussed above, the coexistence is stable in the averaged models. This may appear to show that responsive switching destabilizes coexistence in this case, but permanent 
coexistence [31] of the two species does not require the coexistence equilibrium to be stable. Indeed, we have only discussed steady-state coexistence up to this point, but no steady-state of Eqs. (7) is stable within that (possibly empty) intersection and the dynamics of Eqs. (7) are bounded, so steady-state coexistence cannot give a complete picture of these dynamics. We therefore extend our analytical results beyond the above analysis of the stability of the steady states of Eqs. (7): if $\vartheta+\gamma \iota / \delta<\zeta<\eta / \alpha$ and hence $\mathcal{A}$ and $\mathcal{B}$ are both unstable, then both species coexist permanently, independently of the initial conditions (Appendix C). The mathematical observation that responsive switching destabilizes the coexistence equilibrium at large $\beta$ does not contradict the biological picture of responsive switching stabilizing coexistence that we have painted above: it simply implies that coexistence cannot be at steady state in this case, and hence that responsive switching induces oscillatory population dynamics.

All of this stresses how phenotypic switching increases the complexity of the dynamics: no limit cycles (and no more complex attractors) arise in classical two-species Lotka-Volterra competition model [26]. Thus analysis of the linear stability of the steady states (Ref. [26] and Appendix B) provides a complete biological picture of the community for the average model, but does not yield a similarly complete picture of a two-species community with phenotypic variation.

Classifying all attractors (i.e. not only the steady-state attractors) of Eqs. (7) and the initial conditions that lead to them is beyond the scope of this paper. Our results imply, for example, that unless $\vartheta+\gamma \iota / \delta<\zeta<\eta / \alpha$, at least one of $\mathcal{A}$ and $\mathcal{B}$ is stable, and so there exist initial conditions for which Eqs. (7) converge to $\mathcal{A}$ or $\mathcal{B}$ and hence for which coexistence is not permanent. They cannot, however, reveal, what these specific initial conditions are, or indeed establish or disprove convergence to a non-steady-state attractor where extinction of one species is the only stable equilibrium. In this context, our result that, for $\zeta>\max \{\vartheta+\gamma \iota / \delta, \eta / \alpha\}$, stable steady-state coexistence is possible for sufficiently large $\beta$ merely shows that some initial conditions must converge to stable steadystate coexistence, while these initial conditions must lead to extinction of one species in the absence of phenotypic variation. It is to this extent that we can state that responsive switching is stabilizing at this stage.

While the stability and feasibility of the steady states of the averaged model with stochastic switching only corresponds exactly to that of the averaged model without phenotypic variation, it is not however clear that this correspondence should extend to the dynamics away from equilibrium. Indeed, the nonexistence of non-steady-state attractors in the absence of phenotypic switching [26] does not imply that non-steady-state attractors cannot exist in the model with stochastic switching only. In particular, our analysis leaves open the possibility that these same initial conditions could lead to permanent coexistence (albeit not at steady state) in the averaged model with stochastic switching only and hence that it could be any switching, and not specifically responsive switching, that stabilizes coexistence. We will return to this question when analyzing Eqs. (6). Our numerical results will confirm that responsive switching stabilizes coexistence even compared to the averaged model with stochastic switching only.

\section{Analysis of the simplified model (8)}

The simplified model (8) has three trivial steady states similar to those of Eqs. (7) defined in Eqs. (11a). They are

$$
\mathcal{O}=(0,0,0), \quad \mathcal{A}=(0,0, \zeta / \eta), \quad \mathcal{B}=I(1, \gamma /(\delta-\mu), 0),
$$

wherein $I=1+\gamma \mu /(\delta-\mu)$. It also has as a single coexistence equilibrium,

$$
\mathcal{C}=(b, p, a)
$$

We postpone the detailed calculations that determine these steady states as well as their feasibility and stability to Appendix $\mathrm{D}$, and summarize and discuss the results below. We shall assume throughout that $\delta>\mu$, which is a necessary condition for $\mathcal{C}$ to be feasible (Appendix D), and also a sufficient conditions for the dynamics of Eqs. (8) to be bounded (Appendix A). We may also assume that $\mu>0$ since the case $\mu=0$ is clearly equivalent to the case $\iota=0$ for the simplified model (7) considered above.

In the absence of responsive switching, i.e. if $\beta=0$, coexistence at $\mathcal{C}$ is feasible and stable if and only if the double inequality $\vartheta<\zeta / I<\eta / \alpha$ holds (Appendix D and Fig. 6). As shown in Appendix B, this is exactly the stability condition for the averaged model corresponding to Eqs. (8): again, stochastic switching does not affect the stability of the system.

If $\vartheta<\eta / \alpha$, this region of stable coexistence at $\beta=0$ extends to a corresponding region of stability for $\beta>0$, although coexistence is unstable at large $\beta$ and $\zeta$ [Fig. 6(a)]. The precise extent of this region of instability in the $(\zeta, \beta)$ diagram must be computed numerically. If $\vartheta>\eta / \alpha$, coexistence is always unstable if $\beta=0$, but, as shown in Appendix $\mathrm{D}$, a range of stable coexistence exists for $\beta>\beta_{*}$, where

$$
\beta_{*}>\beta_{*}^{\min }=\frac{\delta-\mu}{\mu \vartheta}(\alpha \vartheta-\eta),
$$

as shown in Fig. 6(b). Again, $\beta_{*}^{\min }=O(\varepsilon)$ is possible even if $\mu=O(\varepsilon)$, provided that $\alpha \vartheta-\eta \lesssim O\left(\varepsilon^{2}\right)$. This is precisely the condition that we obtained when analyzing model (7). A region of instability at large $\beta$ and $\zeta$ also exists for $\vartheta>\eta / \alpha$, as shown in Fig. 6(b). In this case, the region of instability may extend down to the singular point $\zeta=\vartheta I, \beta=\beta_{*}^{\min }$; the conditions for which this occurs, albeit not edifying, are derived in Appendix D.

To assess the effect of responsive switching on the stability of the community for this model, we again compare its stability diagram to that of the corresponding averaged models without phenotypic variation and with stochastic switching only. The calculations in Appendix B determine the range of stability of these averaged models (Fig. 6). These results emphasize the importance of establishing correspondences to averaged models when making these comparative stability statements: the range of $\zeta$ for which coexistence is stable increases with $\beta$ initially [Fig. 6(a)], but the parameters of the averaged models vary correspondingly, and so responsive switching is neither stabilizing nor destabilizing for small $\beta$. However, large levels of responsive switching destabilize $\mathcal{C}$ if $\vartheta<\eta / \alpha$ [Fig. 6(a)]. 

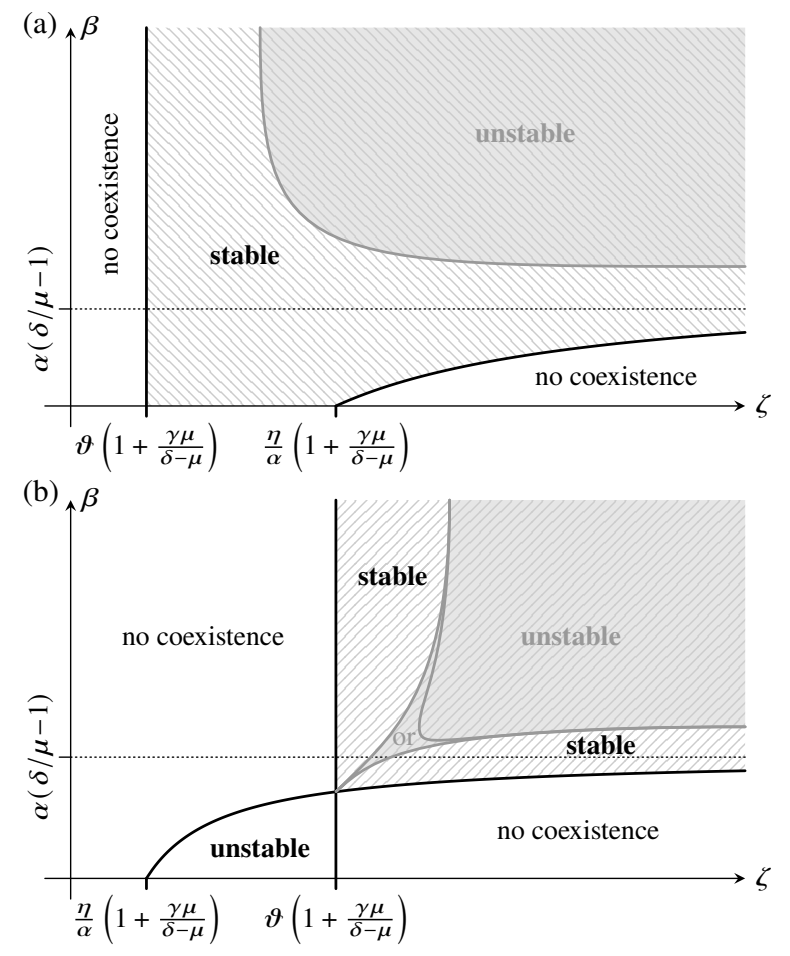

FIG. 6. Feasibility and stability of the coexistence state $\mathcal{C}$ of the simplified model $(8)$ in the $(\zeta, \beta)$ diagram for $\mu \neq 0$, in the cases (a) $\eta / \alpha>\vartheta$ and (b) $\eta / \alpha<\vartheta$. Coexistence is feasible in the region bounded by the thick solid black lines, and is stable or unstable in the regions marked "stable" or "unstable", respectively, as discussed in the text and Appendix D. A region of instability (grey lines) arises at sufficiently large $\beta, \zeta$. The exact boundary of this region must be computed numerically, but the conditions for the alternative behaviors ("or") in panel (b) are derived in Appendix D. In the hatched region of parameter space, the trivial steady states $\mathcal{A}$ and $\mathcal{B}$ are unstable, and the two species coexist permanently (Appendix D). The solidly hatched region in panel (a) is also the region of stable steady-state coexistence for averages of Eqs. (8) without phenotypic variation and with stochastic switching only (Appendix B); the dashed hatching in panel (b) signifies that steady-state coexistence of the averaged models cannot be stable for $\eta / \alpha<\vartheta$ (Appendix B). In the shaded regions of parameter space, all steady states of Eqs. (8) are unstable.

This destabilization of the coexistence equilibrium does not mean, however, that responsive switching destabilizes coexistence: in the region of large $\beta, \zeta$ where $\mathcal{C}$ is feasible, but unstable, the two species still coexist permanently (Appendix D). The fact that all steady states of Eqs. (8) are unstable there simply means that the two species do not coexist at steady state. Similarly, Fig. 6(b) shows that the combination of responsive switching and persister growth is stabilizing if $\vartheta>\eta / \alpha$ and the competitors grow sufficiently fast; again, for large $\beta, \zeta$, the two species cannot coexist at steady state, but do coexist permanently (Appendix D). As already discussed for model (7), this stabilization argument cannot exclude the possibility of permanent, non-steady-state coexistence in the model with stochastic switching only (which is not possible in the averaged model without phenotypic variation). Our numerical results for Eqs. (1) will do so and hence confirm that it is indeed responsive switching that stabilizes coexistence.
The condition for stability of $\mathcal{B}$, in which the competitors are extinct, is $\zeta<\vartheta I$ (Appendix D), so is independent of $\beta$. Again, responsive switching cannot therefore drive the competitors to extinction. However, $\mathcal{A}$, in which the bacteria and persisters are extinct, is stable only if $\beta<\alpha(\delta / \mu-1)$ (Appendix D). Not only does responsive switching thus destabilize $\mathcal{A}$ and hence extinction of bacteria and persisters. It also makes permanent coexistence (albeit not necessarily at steady state) possible in $\zeta>I \max \{\eta / \alpha, \vartheta\}$ (Fig. 6), where extinction of bacteria and persisters is the only possible steady state at $\beta=0$. All of this confirms the picture already suggested by the analysis of model (7), that responsive switching stabilizes coexistence.

\section{Analysis of the simplified model (9)}

Finally, we analyze the simplified model (9). Similarly to model (7), it has three trivial steady states and two coexistence equilibria. They are

$$
\mathcal{O}=(0,0,0), \quad \mathcal{A}=(0,0, \zeta / \eta), \quad \mathcal{B}=J^{-1}(1, \gamma / \delta, 0)
$$

with $J=1+\gamma \kappa / \delta$, and

$$
\mathcal{C}_{+}=\left(b_{+}, p_{+}, a_{+}\right), \quad \mathcal{C}_{-}=\left(b_{-}, p_{-}, a_{-}\right) .
$$

If $\beta \kappa=0$, there is but a single coexistence state, $\mathcal{C}=(b, p, a)$. The expressions for the coordinates of the coexistence equilibria $\mathcal{C}_{+}, \mathcal{C}_{-}$and $\mathcal{C}$ are given in Appendix E, where we also analyze the feasibility and stability of the steady states in detail. We summarize and discuss these results in what follows; we may assume that $\kappa>0$, since the case $\kappa=0$ is clearly equivalent to the case $\iota=0$ in the discussion of model (7).

If there is no responsive switching, i.e. if $\beta=0$, we find that $\mathcal{C}$ is stable if and only if $\vartheta J<\zeta<\eta / \alpha$ (Fig. 7), assuming that the additional inequality $\delta[\delta+\vartheta(\gamma+\delta)]+\gamma \kappa(\delta-\zeta)>0$ holds (Appendix E); this inequality always holds if $\delta>\zeta$, and holds for $\delta<\zeta$ if the persister scalings (10) are satisfied. The condition $\vartheta J<\zeta<\eta / \alpha$ is, as shown in Appendix B, exactly the stability condition for coexistence of the averaged model corresponding to Eqs. (9). Thus, assuming that the condition discussed above holds, stability is once again not affected by stochastic switching.

For $\beta \kappa \neq 0$, the calculations in Appendix E show that both coexistence states are feasible if $\zeta<\min \{\vartheta J, \eta / \alpha\}$ and $\beta$ is sufficiently large, say $\beta>\beta_{*}$ (Fig. 7). We determine $\beta_{*}$ in terms of the other model parameters in closed form in Appendix $\mathrm{E}$, and note here that

$$
\beta_{*}>\beta_{*}^{\min }=\frac{|\delta(\eta-\alpha \vartheta) / \kappa-\gamma \eta|}{\min \{\eta / \alpha, \vartheta /(1+\gamma \kappa / \delta)\}^{2}} .
$$

Once again, $\beta_{*}^{\min }=O(\varepsilon)$ is possible even if $\kappa=O(\varepsilon)$ provided that $\eta-\alpha \vartheta \lesssim O\left(\varepsilon^{2}\right)$. This is the now familiar condition that the intra-species competitions of bacteria and competitors be sufficiently close to their inter-species competitions.

We now turn to discussing the stability of coexistence at $\beta \kappa \neq 0$. One of the coexistence states is always unstable, but the other is stable if it is feasible for sufficiently large $\beta$ 

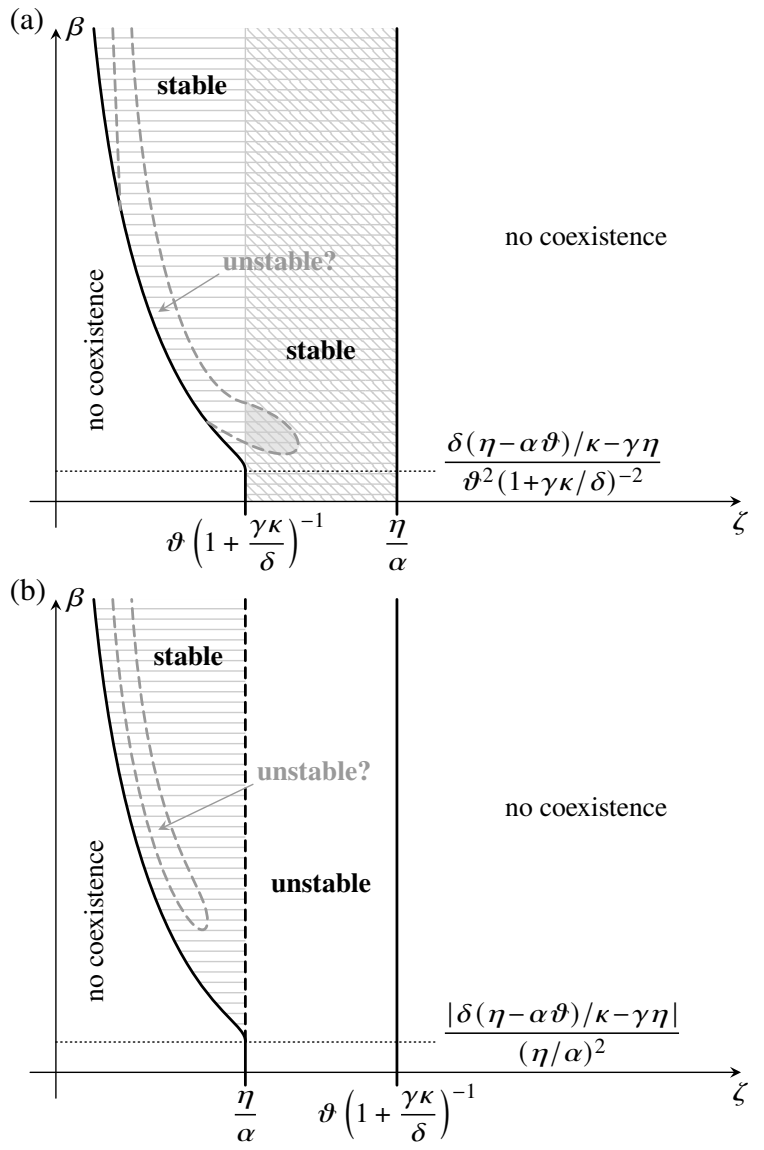

FIG. 7. Feasibility and stability of the coexistence states $\mathcal{C}_{ \pm}$of the simplified model (9) in the $(\zeta, \beta)$ diagram for $\kappa \neq 0$, in the cases (a) $\eta / \alpha>\vartheta /(1+\kappa \gamma / \delta)$ and (b) $\eta / \alpha<\vartheta /(1+\kappa \gamma / \delta)$, assuming the scalings (10). Coexistence is feasible in the region bounded by the thick solid black lines. Both $\mathcal{C}_{+}$and $\mathcal{C}_{-}$are unstable in the region marked "unstable", but $\mathcal{C}_{-}$is stable (and $\mathcal{C}_{+}$is unstable) in the region marked "stable", as discussed in the text and Appendix E. Whether $\mathcal{C}_{-}$is unstable in a subregion of the "stable" region must be determined numerically; if such a subregion exists, it is restricted by conditions derived in Appendix E. In the diagonally hatched region of parameter space, the trivial steady states $\mathcal{A}$ and $\mathcal{B}$ are unstable, and the two species coexist permanently (Appendix E). If the "unstable" region intersects this region, all steady states of Eqs. (7) are unstable within this intersection. The horizontally hatched region is the region of stable steady-state coexistence for averaged models of Eqs. (9) without phenotypic variation and with stochastic switching only (Appendix B).

(Appendix E and Fig. 7). Under the additional assumption that $\vartheta>\kappa$, which is consistent with the persister scalings (10), the calculations in Appendix E restrict possible subregions of instability within the domain of feasibility, as shown in Fig. 7. Numerically, we found such subregions for $\vartheta<\eta / \alpha$, but did not find any for $\vartheta>\eta / \alpha$. We were not however able to prove that such subregions do not exist in the latter case, nor did we obtain any analytical results establishing conditions for the existence or absence of such subregions.

Finally, we compare once again the stability diagram of this simplified model with responsive switching to the conditions for stability (Appendix B) of the corresponding averaged mod- els without phenotypic variation and with stochastic switching only. The results of this comparison, shown in Fig. 7, stress again the importance of careful model averaging: although the region of parameter space in which coexistence is stable widens with increasing $\beta$, the corresponding variation of the model parameter means that coexistence is stable in the averaged model if and only if it is feasible. Thus, if regions of instability as discussed above exist, steady-state coexistence is destabilized there by the combination of responsive switching and competition between bacteria and persisters. Again, this argument does not preclude non-steady coexistence there. Indeed, the two species coexist permanently if $\vartheta / J<\zeta<\eta / \alpha$ even if no coexistence state is stable (Appendix E), but our analytical results do not reveal whether the dynamics converge to (non-steady-state) attractors in other regions of parameter space in which no coexistence state is stable. Anyway, as discussed below, numerical results for the stability of Eqs. (9) suggest that such regions of instability are rare for parameter values consistent with the persister scalings (10).

\section{Full two-species model: numerical study}

In this subsection, we extend the exact results for the simplified models (7), (8), (9) obtained in the previous subsection to the full model (6), which we study numerically. As in our analysis of these simplified models, the question that we ask for the full model is: how and to what extent does responsive switching stabilize coexistence?

\section{Numerical setup}

Although the full model (6) is too complicated for meaningful analytical progress to be made, its equilibria can be found numerically and efficiently by precomputing, using MATHEMATICA, the exact polynomial equations satisfied by the equilibria from Gröbner bases [34]. For each computed equilibrium, we check its accuracy using the values of the right-hand sides of Eqs. (6) evaluated there, and verify that all solutions have been found using a test based on Sturm's theorem [35]. Finally, we determine the stability of the computed equilibria using the Routh-Hurwitz conditions [26]. Similarly, we determine the stability of equilibria of the averaged models (with stochastic switching only and without phenotypic variation) corresponding to Eqs. (6). Unstable equilibria are integrated numerically, similarly to the calculations in Sec. II, to determine whether the species coexist permanently notwithstanding a particular coexistence equilibrium being unstable. We reduce the number of systems that need to be integrated in this way by noting that our result for the simplified models (7), (8), (9), that coexistence is permanent if all trivial steady states are unstable, extends to the full model (6) if the persister scalings (10) are satisfied (Appendix F).

The setup for our numerical calculations is as follows: we fix a population of bacteria and competitors by fixing the competition parameters $\alpha, \eta, \vartheta$, and compute stability diagrams in the $(\zeta, \beta)$ plane for random choices of the remaining model parameters $\gamma, \delta, \iota, \kappa, \mu, \xi, \varpi, \varsigma$, which we constrain to satisfy the 
(a)

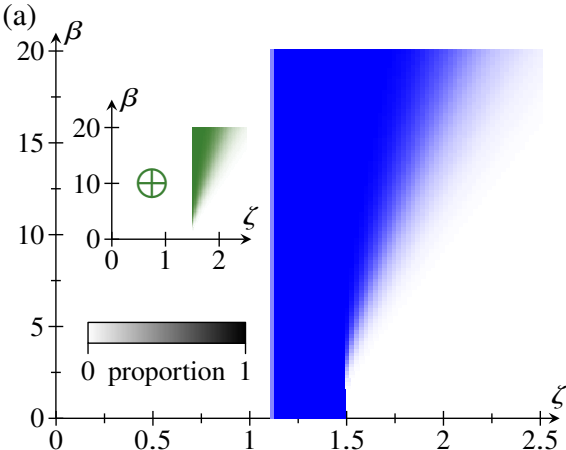

(b)

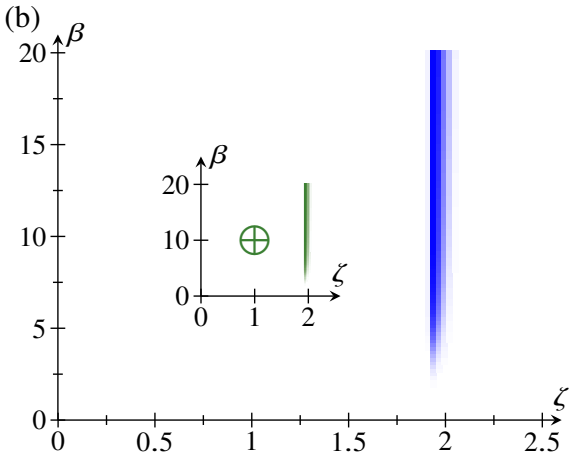

(c)

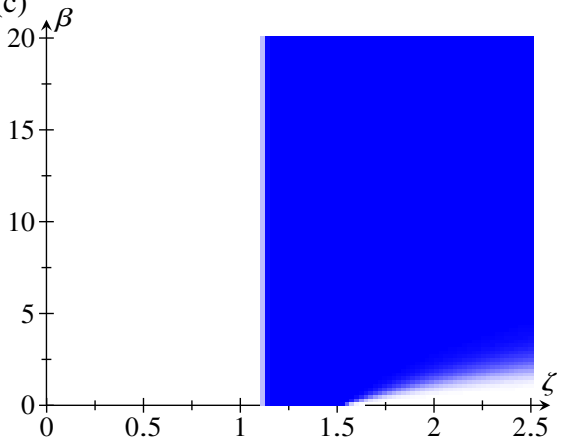

(d)

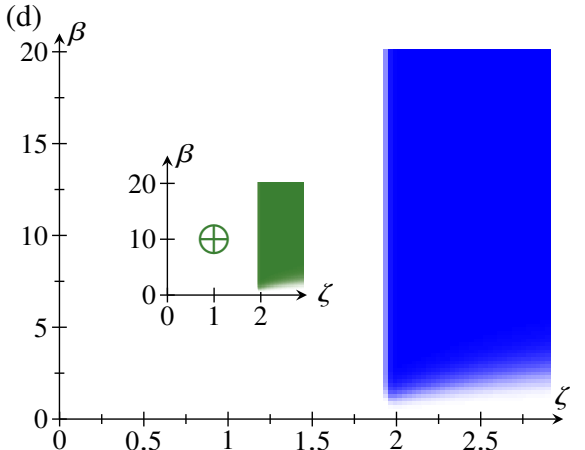

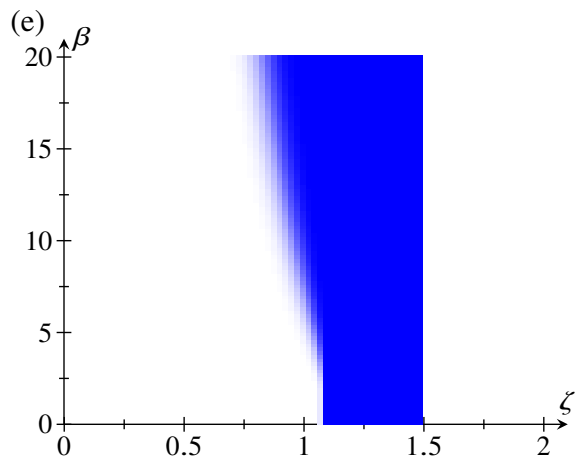

(f)

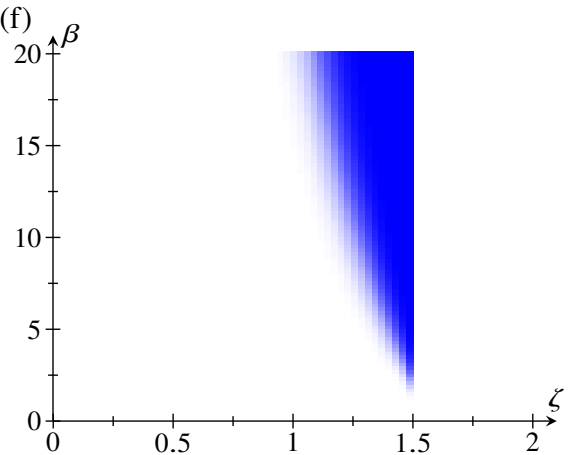

FIG. 8. Effect of the parameters $\iota, \mu, \kappa$ on the stability and permanence of coexistence. Numerical results in the $(\zeta, \beta)$ diagram for the simplified models (7) [panels (a), (b)], (8) [panels (c), (d)], (9) [panels (e), (f)], confirming the analytical results (Figs. 5, 6, 7, respectively). The cases $\eta / \alpha>\vartheta$ [panels (a), (c), (e)] and $\eta / \alpha<\vartheta$ [panels (b), (d), (f)] lead to qualitatively different diagrams. The color of each point in the stability diagrams represents the proportion of $N=1000$ random systems for which coexistence is stable or permanent at that point. The insets similarly plot the proportion of systems for which coexistence is stabilized (or becomes permanent), $\oplus$, or destabilized, $\ominus$, compared to the averaged model with stochastic switching only; there is no (de)stabilization where there is no inset. Parameter values: $\alpha=0.8, \eta=1.2$, and $\vartheta=1.1$ [panels (a), (c), (e)] or $\vartheta=1.9$ [panels (b), (d), (f)]. The remaining parameters (if not set to zero) were sampled uniformly and independently, constrained by the persister scalings (10): $\gamma, \iota, \kappa, \mu, \xi, \varpi, \varsigma \sim \mathcal{U}[\varepsilon, 2 \varepsilon]$, with $\varepsilon=0.1$, and $\delta \sim \mathcal{U}[0.8,1.6]$.
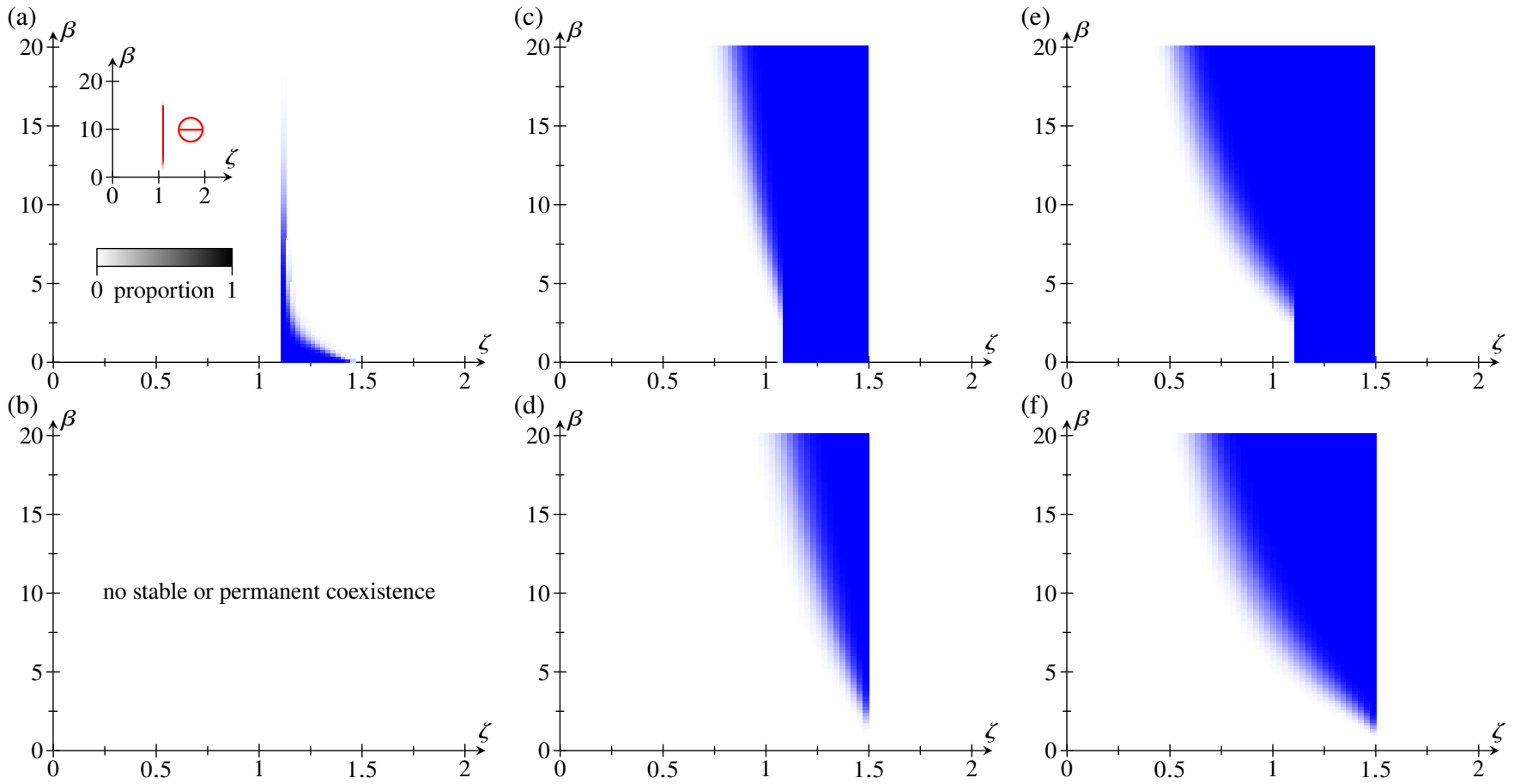

FIG. 9. Effect of the parameters $\xi, \varpi, \varsigma$ on the stability and permanence of coexistence. Numerical stability and permanence results for the full model (6) in the $(\zeta, \beta)$ plane, analogous to those in Fig. 8, but now in the cases $\xi \neq 0, \iota=\kappa=\mu=\varpi=\varsigma=0$ [panels (a), (b)], $\varpi \neq 0, \iota=\kappa=\mu=\xi=\varsigma=0$ [panels (c), (d)], and $\varsigma \neq 0, \iota=\kappa=\mu=\xi=\varpi=0$ [panels (e), (f)]. Panels (a), (c), (e) have $\eta / \alpha>\vartheta$, while panels (b), (d), (f) have $\eta / \alpha<\vartheta$. Further explanations and parameter values are in the caption of Fig. 8 . 
persister scalings (10). We report these numerical results by plotting, for each point in the $(\zeta, \beta)$ diagram, stability statistics (such as the proportion of random systems in which coexistence is stable or permanent at this point), but it is important to note that these statistics, while providing a convenient way of visualizing the generic properties of the stability diagrams, have no real biological meaning because the averaged models vary between these random instantiations of the full model, since the bacteria-persister competition parameters are held fixed while the switching parameters and the remaining competition parameters are varied.

\section{Results}

We begin by confirming the analytical results of the previous subsection for the effect of the parameters $\iota, \mu, \kappa$. Figure 8 plots numerical stability diagrams for the simplified models (7), (8), (9). These demonstrate, in agreement with the stability diagrams in Figs. 5 and 6, the stabilizing effect of persister-competitor interactions $[\iota \neq 0$, Figs. 8(a),(b)] and persister growth $[\mu \neq 0$, Figs. 8(c),(d)] compared to the model with stochastic switching only. We note the absence, in Figs. 8(a),(b), of signatures of the regions of instability that arise for Eqs. (7) at large $\beta, \zeta$ (Fig. 7): this implies that, with responsive switching, coexistence is permanent there for initial conditions close to the unstable coexistence equilibrium even though the trivial steady states are not necessarily all unstable. This emphasizes that linear stability of equilibria does not give a complete picture of species coexistence in these models. Analogously, there is no signature either of the possible regions of instability for model (9), sketched in Fig. 7, in the numerical stablity diagrams [Figs. 8(e),(f)]. In fact, numerical experiments (not shown) suggest that such regions are rare for parameter values consistent with the persister scalings (10), but we have no analytical understanding of this. The destabilizing effect of competition between bacteria and persisters $(\kappa \neq 0)$ thus appears to be weaker than the stabilizing effect of persister-competitor interactions and persister growth.

We extend these results to the parameters $\xi, \varpi, \varsigma$ by plotting, in Fig. 9, numerical stability diagrams of the full model (6) for the cases in which one of $\xi, \varpi, \varsigma$ is the only nonzero parameter among the logistic parameters involving persisters, i.e. in the cases $\iota=\kappa=\mu=\varpi=\varsigma=0, \iota=\kappa=\mu=\xi=\varsigma=0$, $\iota=\kappa=\mu=\xi=\varpi=0$. For the generic parameter values used, Figs. 9(a),(b) show a very slight destabilizing effect of competitor-persister interactions $(\xi \neq 0)$. By contrast, for these parameter values, the competition between bacteria and persisters expressed by $\varpi \neq 0$ [Figs. 9(c),(d)] and $\varsigma \neq 0$ [Figs. 9(e),(f)] does not affect stability. Indeed, it is perhaps not suprising that the stability diagrams for these parameters qualitatively match those for the other bacteria-persister competition parameter, viz. $\kappa$, in Figs. 8(e),(f).

The insets in the panels of Figs. 8 and 9 compare the stability (or permanence of coexistence) of model (7) to its average with stochastic switching only, but the numerical results for the comparison of model (7) to its average without phenotypic variation are identical to these. Thus, our numerical results not only confirm that it is indeed responsive switching that stabilizes coexistence (where our analytical results could not preclude non-steady-state coexistence with stochastic switching only). They also extend our analytical result, that stochastic switching on its own does not affect the stability of steady-state coexistence for the simplified models (7), (8), (9), to the statement that stochastic switching on its own does not affect the permanence of coexistence for these models.

These results show that, of the six parameters $\iota, \mu, \kappa, \xi, \varpi, \varsigma$, only the first two have, on their own, a strong effect on stability or permanence of coexistence when coupled to responsive switching; stochastic switching only does not affect stability. The stability problem for Eqs. (6) is not, of course, a linear
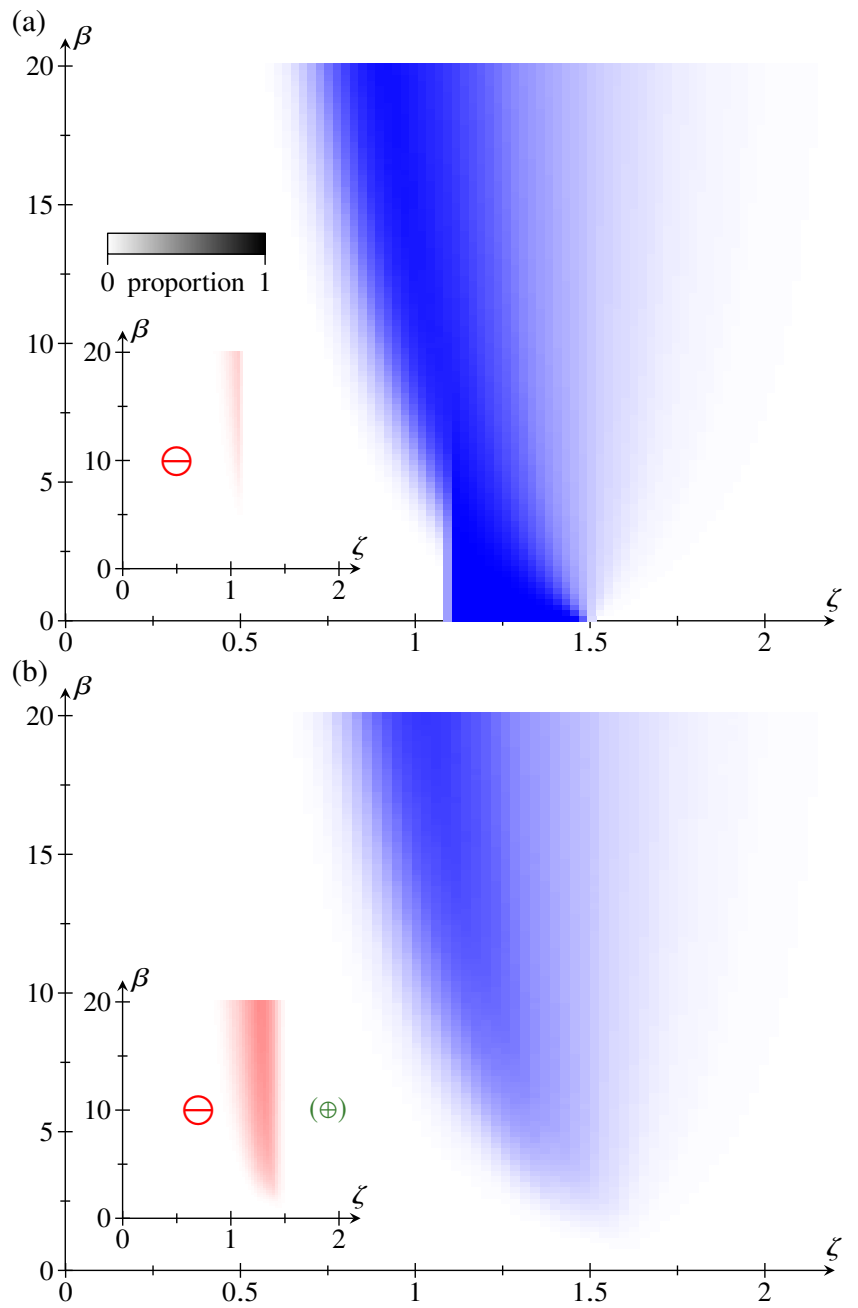

FIG. 10. Numerical stability diagrams of the full model (6) in the $(\zeta, \beta)$ diagram, for $\iota, \kappa, \mu, \xi, \varpi, \varsigma \neq 0$, in the cases (a) $\eta / \alpha>\vartheta$ and (b) $\eta / \alpha<\vartheta$. As in Figs. 8, 9, the color of each point in the stability diagrams represents the proportion of $N=1000$ random systems for which coexistence is stable or permanent at that point and the insets are plots of the proportion of systems for which coexistence is destabilized, $\ominus$, compared to the averaged model with stochastic switching only. The symbol $\oplus$ in parentheses in the inset of panel (b) indicates that a nonzero proportion of systems (too small to be visualizable by the color scheme) is stabilized (or becomes permanent) compared to the averaged model. Parameter values are as in Figs. 8, 9: $\alpha=0.8, \eta=1.2, \vartheta=1.1$ [in panel (a)] or $\vartheta=1.9$ [in panel (b)], $\gamma, \iota, \kappa, \mu, \xi, \varpi, \varsigma \sim \mathcal{U}[\varepsilon, 2 \varepsilon]$, with $\varepsilon=0.1$, and $\delta \sim \mathcal{U}[0.8,1.6]$. 
problem, and so their general stability diagram is not a trivial superposition of the stability diagrams in Figs. 8 and 9.

Nonetheless, we recognize features of the diagrams in Figs. 8 and 9 in the full stability diagrams of Eqs. (6) shown in Fig. 10. It is striking however that sufficient levels of responsive switching destabilize coexistence for the same parameter values for which responsive switching stabilized coexistence or did not affect stability in the "one-parameter" cases considered in Figs. 8 and 9. This destabilizing effect is less pronounced for $\vartheta<\eta / \alpha$ [Fig. 10(a)] than for $\vartheta>\eta / \alpha$ [Fig. 10(b)].

This destabilization is of course that which we already noted when discussing the statistical stability of responsive switching in the previous section: Figure 2(c) has already shown that coexistence is more likely to be stable in models with stochastic switching only than in those with responsive switching. However, the present discussion adds to this in that Eqs. (6), and in particular the simplified models (7), (8), (9), have allowed us to identify those parameters that are stabilizing in combination with responsive switching, which we could not have done for the full model (1). These results do not therefore contradict each other.

What is more, these considerations suggest that coexistence can be stabilized by tuning the persister parameters so that the stabilizing parameters $\iota, \mu$ exceed the others, while still remaining "small", as required by the persister scalings (10). This is confirmed by the numerical results in Fig. 11. The stabilizing effect of "elevating" $\iota, \mu$ is less pronounced for $\vartheta<\eta / \alpha$ [Fig. 11(a)] than for $\vartheta>\eta / \alpha$ [Fig. 11(b)], mirroring the weaker destabilizing effect of "unelevated" $\iota, \mu$ in the former case.

The result for the simplified models (7), (8), (9), that stochastic switching on its own does not affect the permanence of coexistence, does not carry over to the full model (1): the (de)stabilization diagrams for the comparison of Eqs. (1) and its average without phenotypic variation (not shown) are different from those in Figs. 10 and 11. In particular, we conclude from these numerical results that elevated $\iota, \mu$ (Fig. 11) also stabilize the averaged model with stochastic switching only compared to that without phenotypic variation.

Our analysis is based on a weakly-competing, persister-like phenotype P [Fig. 1(b)]. In this context, the "elevated" growth rate $\mu$ that stabilizes coexistence with responsive switching might correspond to a phenotype that relies on a different food source (and therefore competes weakly with both the normal phenotype $\mathrm{B}$ and the competitors A); the growth rate $\mu$ may still be small because the alternative food source may be scarce, or more difficult to metabolize. Similarly, an elevated persister-competitor interaction rate $\iota$ may result from toxins produced by the persisters and acting on the competitors. That the ecological fitness of the phenotypes we have just described should be linked to responsive rather than stochastic switching is perhaps not surprising.

We conclude our discussion by commenting on the values $\beta=O(1)$ used in these numerical calculations and that are not consistent with the persister scalings (10). Our analytical results showed that small rates of stochastic switching, $\beta=O(\varepsilon)$ do affect stability, but only if the B and A phenotypes are similar enough, as expressed by the scaling requirement $\eta-\alpha \vartheta \lesssim O\left(\varepsilon^{2}\right)$. Our choice of allowing "large" values of (a)
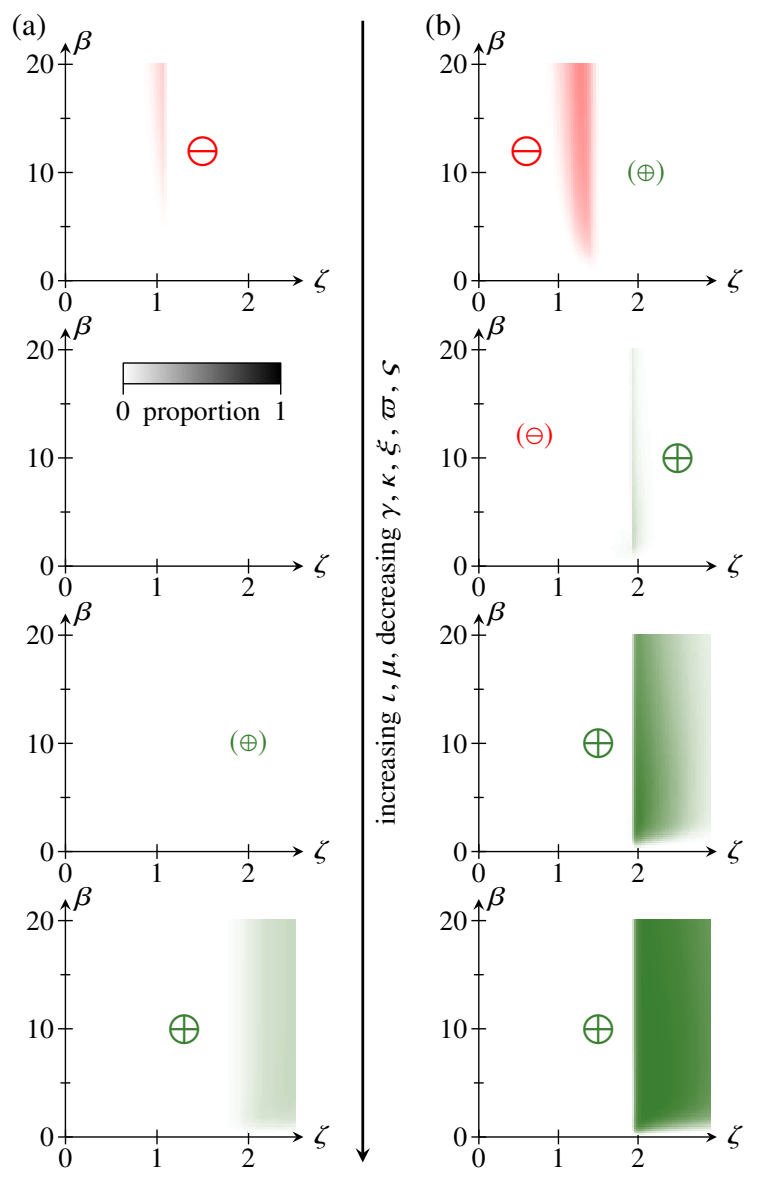

FIG. 11. Stabilization of coexistence by "elevated" persister growth $\mu$ or competitor-persister interaction $\iota$. Plots, in $(\zeta, \beta)$ space and for (a) $\eta / \alpha>\vartheta$ and (b) $\eta / \alpha<\vartheta$, of the proportion of $N=1000$ random instantiations of the full model (6) for which coexistence is stabilized (or becomes permanent), $\oplus$, or destabilized, $\ominus$, compared to the averaged model with stochastic switching only. Proportions are represented by the colors of the points in the diagrams, with symbols in parentheses indicating that the stability or permanence of a small proportion of systems too small to be visualizable by the color scheme changes compared to the averaged model. The "elevation" of $\iota, \mu$ increases from top to bottom. Parameter values: $\alpha=0.8$, $\eta=1.2$, (a) $\vartheta=1.1$ or (b) $\vartheta=1.9$, and $\delta \sim \mathcal{U}[0.8,1.6]$, as in Figs. 8-10; also, $\gamma, \mu, \xi, \varpi, \varsigma \sim \mathcal{U}[\varepsilon / f, 2 \varepsilon / f], \mu, \iota \sim \mathcal{U}[\varepsilon f, 2 \varepsilon f]$, with $\varepsilon=0.1$ and where $f \in\{1$ (top), 1.5,2,2.5 (bottom) $\}$ expresses the "elevation" of $\iota, \mu$.

$\beta$ is therefore one of numerical convenience that does not affect the biological validity of our analysis. The existence of such a requirement is not surprising: an asymptotically rare phenotype $\mathrm{P}$ should not change the stability of communities in which one of the A and B phenotypes strongly dominates the other, $\eta-\alpha \vartheta=O(1)$. Anyway, this case of strong dominance is less relevant to microbial communities, since one does not expect the evolutionary pressure that would lead to such a strong dominance to exist in these systems. In this context, our earlier results in Sec. II and in Ref. [15], that such a rare phenotype can break this asymptoticity as the number of species increases, might be relatable to a combinatorial and statistical increase of species interactions lacking such dominance. 


\section{CONCLUSION}

In this paper, we have analyzed the ecological implications of phenotypic variation and, in particular, response phenotypic switching. We have shown how the statistical coexistence properties of models with responsive phenotypic switching differ from those of corresponding averaged models without phenotypic variation of with stochastic phenotypic switching only. This analysis led us to emphasize the need to define these averaged models carefully. Although this statistical analysis showed that models with responsive switching are less likely to be stable than those with stochastic switching only, exact results for simplified models revealed those parameters (and hence the ecological conditions) in combination with which responsive switching can stabilize two-species communities even when stochastic switching on its own does not affect the stability of the community. Additionally, our numerical results emphasized the importance of non-steady-state attractors for coexistence of all species, even for the simplified two-species models, but analytical understanding of these attractors is still lacking. All of our results thus hint at a complex relationship between ecological stability and phenotypic variation, of which this and previous studies [14, 15] have only scratched the surface.

Extending these results from two-species to many-species systems remains an important challenge for future work. In particular, to understand which phenotypic interactions stabilize or destabilize many-species systems, further analytical progress, for simple models of communities of more than two species, will be crucial to guide statistical exploration of manyspecies systems due to the quadratic increases of the number of interaction parameters with the number of species. Moreover, now that a range of stability-affecting structures of microbial communities, from phenotypic variation (this work and Refs. $[14,15])$ and species modularity [6] to higher-order interactions [7] or resource and signalling dynamics [8, 29], has been identified, further theoretical work will need to analyze the interplay of such effects and ask: Which of these effects (if any) dominates the overturning of May's stability paradigm [1] in actual ecological communities?

Meanwhile, identifying experimental systems in which the questions that we have answered theoretically in this work can be addressed experimentally is another key challenge for future work. In particular, experimental systems for these questions must allow disentangling the effect of responsive phenotypic switching, say, and the effect of the change of the equilibrium population that results from "turning off" responsive switching. This is of course precisely the problem that we highlighted when discussing reduced models and solved, theoretically, by defining averaged models for stability comparison. The ability to make complex, albeit mathematical ecological perturbations that we have exploited by introducing these averaged models into our analysis is fundamentally what explains the power of theoretical approaches for guiding experimental exploration of problems in ecology. It is for this reason that the importance of these theoretical approaches mirrors the outstanding importance of microbial communities [36] — and hence that of understanding the physical and mathematical effect that stabilize or destabilize them-for the world that surrounds us.

\section{ACKNOWLEDGMENTS}

We thank B. G. Stokell for a discussion of confidence intervals. This work was supported in part by the Schlumberger Chair Fund. R.E.G. was supported by Established Career Fellowship No. EP/M017982/1 from the Engineering and Physical Sciences Research Council and Grant No. 7523 from the Marine Microbiology Initiative of the Gordon and Betty Moore Foundation. N.M.O. was supported by Discovery Fellowship No. BB/T009098/1 from the Biotechnology and Biological Sciences Research Council, a Wellcome Trust Interdisciplinary Fellowship, and, at earlier stages of this work, a Herchel Smith Postdoctoral Research Fellowship. P.A.H. was supported by the Max Planck Society, and, at earlier stages of this work, by a Nevile Research Fellowship from Magdalene College, Cambridge and a Hooke Research Fellowship at the Mathematical Institute, University of Oxford.

\section{APPENDIX A: ANALYSIS OF EQS. (1): BOUNDED DYNAMICS, SAMPLING OF RANDOM SYSTEMS, EQUILIBRIA, AND COEXISTENCE}

This Appendix provides the conditions under which the dynamics of Eqs. (1) are bounded, discusses the random sampling of systems, and also gives the details of the calculations of the Jacobians of the equilibria of Eqs. (1) and of the asymptotic calculations in the limit $\varepsilon \ll 1$.

\section{Bounded Dynamics of Eqs. (1)}

In this section, we give sufficient conditions for the dynamics of Eqs. (1) to be bounded, and therefore to be realistic biologically.

We assume that, for each $n \in\{1,2, \ldots, N\}$, the B phenotype of species $n$ satisfies $C_{n n} \neq 0$ or $b_{n}<\varepsilon k_{n}$, and that its $\mathrm{P}$ phenotype satisfies $F_{n n} \neq 0$ or $p_{n}<\ell_{n}$. Then, from Eqs. (1),

$$
\begin{aligned}
\dot{B}_{n}+\dot{P}_{n} \leqslant B_{n} & {\left[\left(b_{n}-\varepsilon k_{n}\right)-C_{n n} B_{n}\right] } \\
& +\varepsilon P_{n}\left[\left(p_{n}-\ell_{n}\right)-F_{n n} C_{n}\right] .
\end{aligned}
$$

Consider first the generic case in which $C_{n n}, F_{n n} \neq 0$. Then $\dot{B}_{n}+\dot{P}_{n}<0$ if $B_{n}>b_{n} / C_{n n}$ and $P_{n}>p_{n} / F_{n n}$. Moreover, if $P_{n}<p_{n} / F_{n n}$ or $B_{n}<b_{n} / C_{n n}$, then from Eqs. (1),

$$
\begin{aligned}
\dot{B}_{n} & \leqslant B_{n}\left(b_{n}-\varepsilon k_{n}\right)-C_{n n} B_{n}^{2}+\ell_{n} P_{n} \\
& <B_{n}\left(b_{n}-\varepsilon k_{n}\right)-C_{n n} B_{n}^{2}+\ell_{n} p_{n} / F_{n n}, \\
\dot{P}_{n} & \leqslant \varepsilon\left[P_{n}\left(p_{n}-\ell_{n}\right)-F_{n n} C_{n}^{2}+k_{n} B_{n}\right] \\
& <\varepsilon\left[P_{n}\left(p_{n}-\ell_{n}\right)-F_{n n} C_{n}^{2}+k_{n} b_{n} / C_{n n}\right] .
\end{aligned}
$$

It follows that $\dot{B}_{n}<0$ if $P_{n}<p_{n} / F_{n n}$ and $B_{n}>B_{n}^{\min }$, for some $B_{n}^{\min }>0$. We will not need an explicit expression for $B_{n}^{\min }$, but can assume without loss of generality that $B_{n}^{\min }>b_{n} / C_{n n}$; similarly, $\dot{P}_{n}<0$ if $B_{n}<b_{n} / C_{n n}$ and $P_{n}>P_{n}^{\min }$, for some $P_{n}^{\min }>p_{n} / F_{n n}$. These bounds are independent of the other species, and thus show that, irrespective of the initial conditions, the dynamics of $\left(B_{n}, P_{n}\right)$ will enter the bounded region in Fig. 12, and remain in that region. 


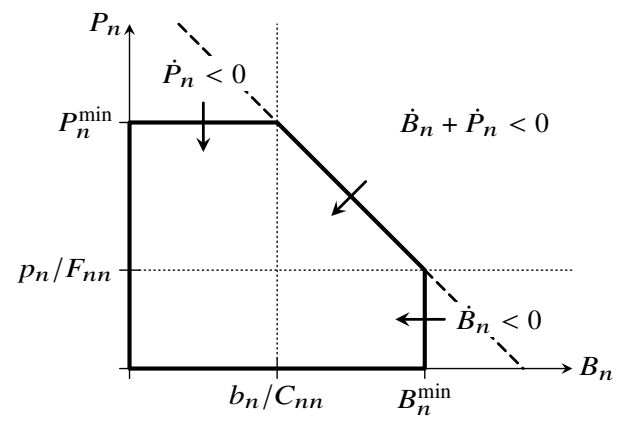

FIG. 12. Bounded dynamics of Eqs. (1). For any nonnegative initial conditions, $\left(B_{n}, P_{n}\right)$ will eventually enter the pentagonal bounding region (thick lines), and remain in that region for all times. If $C_{n n}=0$ and $b_{n}<\varepsilon k_{n}$ or $F_{n n}=0$ and $p_{n}<\ell_{n}$, the sides of the bounding region are modified (dashed lines).

A similar argument shows that the dynamics are bounded if $C_{n n}=0$, but $b_{n}<\varepsilon k_{n}$ or $F_{n n}=0$, but $p_{n}<\ell_{n}$ (Fig. 12).

On identifying parameters appropriately, these conditions also provide sufficient conditions for the dynamics of the twospecies model (6), or the simplified models (7), (8), (9), to be bounded.

\section{Sampling of Random Systems}

We follow the approach that we introduced in Ref. [15] to sample random instantiations of Eqs. (1) and their coexistence equilibria.

In more detail, we choose the competition strengths $\mathrm{C}, \mathrm{D}, \mathrm{E}, \mathrm{F}$, the stochastic switching rates $\boldsymbol{k}, \boldsymbol{\ell}$, the responsive switching rates $\mathrm{R}, \mathrm{S}$, and the equilibria $\boldsymbol{B}_{*}, \boldsymbol{P}_{*}$ independently from the uniform $\mathcal{U}[0,1]$ distribution. This leaves linear equations to be solved for the remaining parameters $\boldsymbol{b}, \boldsymbol{p}$; to ensure that they are nonnegative, we choose a common random scaling for the switching rates $\boldsymbol{k}, \boldsymbol{\ell}$ and $\mathrm{R}, \mathrm{S}$.

Using the asymptotic solution derived below, the model parameters can be sampled directly, i.e. we can sample $\boldsymbol{b}, \boldsymbol{p}$ randomly, and use that solution to compute $\boldsymbol{B}_{*}, \boldsymbol{P}_{*}$. To avoid a breakdown of asymptoticity, we sample parameters in the interval $\left[\varepsilon^{1 / 4}, 1\right]$ rather than $[0,1]$. We also discard those sampled systems for which any component of the right-hand sides of Eqs. (1) is greater $\varepsilon$. Moreover, we ensure feasibility of $\boldsymbol{B}_{*}$ by sampling $\boldsymbol{b}$ as a linear combination of the (normalized) columns of $\mathrm{C}$.

Finally, to sample exact equilibria (again indirectly) for $\varepsilon \ll 1$, we adapt our previous strategy by imposing $\boldsymbol{P}=\varepsilon \boldsymbol{B}(\boldsymbol{k}+\mathrm{R} \cdot \boldsymbol{B})$, up to a random $O\left(\varepsilon^{2}\right)$ correction, to ensure that $\boldsymbol{p}=O(1)$.

\section{Jacobian of Equilibria of Eqs. (1)}

The Jacobian of an equilibrium $\mathcal{E}=\left(\boldsymbol{B}_{*}, \boldsymbol{P}_{*}\right)$ of Eqs. (1) is

$$
J_{*}=\left(\begin{array}{c|c}
J_{1} & J_{2} \\
\hline J_{3} & J_{4}
\end{array}\right)
$$

wherein

$$
\begin{aligned}
\mathrm{J}_{1}= & \left(\boldsymbol{b}-\mathrm{C} \cdot \boldsymbol{B}_{*}-\varepsilon \mathrm{D} \cdot \boldsymbol{P}_{*}\right) \mathrm{I}-\boldsymbol{B}_{*} \mathrm{C} \\
& \quad-\varepsilon\left(\boldsymbol{k}+\mathrm{R} \cdot \boldsymbol{B}_{*}+\mathrm{S} \cdot \boldsymbol{P}_{*}\right) \mathrm{I}-\varepsilon \boldsymbol{B}_{*} \mathrm{R}, \\
\mathrm{J}_{2}= & -\varepsilon \boldsymbol{B}_{*} \mathrm{D}+\boldsymbol{\ell} \mathrm{I}-\varepsilon \boldsymbol{B}_{*} \mathrm{~S} \\
\mathrm{~J}_{3}= & -\varepsilon \boldsymbol{P}_{*} \mathrm{E}+\varepsilon\left(\boldsymbol{k}+\mathrm{R} \cdot \boldsymbol{B}_{*}+\mathrm{S} \cdot \boldsymbol{P}_{*}\right) \mathrm{I}+\varepsilon \boldsymbol{B}_{*} \mathrm{R}, \\
\mathrm{J}_{4}= & \varepsilon\left(\boldsymbol{c}-\mathrm{E} \cdot \boldsymbol{B}_{*}-\varepsilon \mathrm{F} \cdot \boldsymbol{P}_{*}\right) \mathrm{I}-\varepsilon \boldsymbol{P}_{*} \mathrm{~F}-\boldsymbol{\ell} \mathrm{I}+\varepsilon \boldsymbol{B}_{*} \mathrm{~S},
\end{aligned}
$$

with I being the identity. Using $\boldsymbol{k}^{\prime}=\boldsymbol{k}+\mathrm{R} \cdot \boldsymbol{B}_{*}+\mathrm{S} \cdot \boldsymbol{P}_{*}$, Eq. (5) follows immediately.

\section{Asymptotic Coexistence Equilibria of Eqs. (1) for $\varepsilon \ll 1$}

As announced in the main text, we seek an expansion of the coexistence state $\left(\boldsymbol{B}_{*}, \boldsymbol{P}_{*}\right)$ in powers of $\varepsilon \ll 1$ by writing

$$
\boldsymbol{B}_{*}=\boldsymbol{B}_{0}+\varepsilon \boldsymbol{B}_{\mathbf{1}}+O\left(\varepsilon^{2}\right), \quad \boldsymbol{P}_{*}=\boldsymbol{P}_{\mathbf{0}}+\varepsilon \boldsymbol{P}_{\mathbf{1}}+O\left(\varepsilon^{2}\right) .
$$

On expanding Eqs. (1), we find

$$
\begin{aligned}
& \mathbf{0}=\boldsymbol{B}_{\mathbf{0}}\left(\boldsymbol{b}-\mathrm{C} \cdot \boldsymbol{B}_{\mathbf{0}}\right)+\boldsymbol{\ell} \boldsymbol{P}_{\mathbf{0}}+\varepsilon\left[\boldsymbol{B}_{\mathbf{1}}\left(\boldsymbol{b}-\mathrm{C} \cdot \boldsymbol{B}_{\mathbf{0}}\right)-\boldsymbol{B}_{\mathbf{0}} \mathrm{C} \cdot \boldsymbol{B}_{\mathbf{1}}\right. \\
& \left.-\boldsymbol{B}_{0} \mathrm{D} \cdot \boldsymbol{P}_{\mathbf{0}}+\boldsymbol{\ell} \boldsymbol{P}_{1}-\boldsymbol{B}_{0}\left(\boldsymbol{k}+\mathrm{R} \cdot \boldsymbol{B}_{0}+\mathrm{S} \cdot \boldsymbol{P}_{0}\right)\right]+O\left(\varepsilon^{2}\right), \\
& \mathbf{0}=-\boldsymbol{\ell} \boldsymbol{P}_{\mathbf{0}}+\varepsilon\left[\boldsymbol{P}_{\mathbf{0}}\left(\boldsymbol{p}-\mathrm{E} \cdot \boldsymbol{B}_{\mathbf{0}}-\mathrm{F} \cdot \boldsymbol{P}_{\mathbf{0}}\right)-\boldsymbol{\ell} \boldsymbol{P}_{\mathbf{1}}\right. \\
& \left.+\boldsymbol{B}_{0}\left(\boldsymbol{k}+\mathrm{R} \cdot \boldsymbol{B}_{0}+\mathrm{S} \cdot \boldsymbol{P}_{0}\right)\right]+O\left(\varepsilon^{2}\right) .
\end{aligned}
$$

Solving at order $O\left(\varepsilon^{0}\right), \boldsymbol{P}_{\mathbf{0}}=\mathbf{0} \Longrightarrow \boldsymbol{B}_{\mathbf{0}}=\mathrm{C}^{-1} \cdot \boldsymbol{b}$, unless $\operatorname{det} C=0$, which we assume not to be the case. Then, at order $O\left(\varepsilon^{1}\right), \boldsymbol{P}_{\mathbf{1}}=\left(\boldsymbol{k}+\mathrm{R} \cdot \boldsymbol{B}_{\mathbf{0}}\right) \boldsymbol{B}_{\mathbf{0}} / \boldsymbol{\ell}$, and hence $\boldsymbol{B}_{\mathbf{0}} \mathrm{C} \cdot \boldsymbol{B}_{\mathbf{1}}=\mathbf{0}$, which implies $\boldsymbol{B}_{\mathbf{1}}=\mathbf{0}$, as claimed in the main text. On substituting these results into Eq. (A3), we find

$$
\begin{aligned}
& J_{*}=\underbrace{\left(\begin{array}{c|c|c}
-\boldsymbol{B}_{\mathbf{0}} \mathrm{C} & \boldsymbol{\ell} \mathrm{I} \\
\hline \mathrm{O} & -\boldsymbol{\ell} \mid
\end{array}\right)+\varepsilon\left(\begin{array}{c|c}
-\left(\boldsymbol{k}+\mathrm{R} \cdot \boldsymbol{B}_{\mathbf{0}}\right) \mathrm{I} & -\boldsymbol{B}_{\mathbf{0}} \mathrm{D} \\
\hline\left(\boldsymbol{k}+\mathrm{R} \cdot \boldsymbol{B}_{\mathbf{0}}\right) \mid & \left(\boldsymbol{p}-\mathrm{E} \cdot \boldsymbol{B}_{\mathbf{0}}\right) \mathrm{I}
\end{array}\right)}_{\mathrm{K}_{*}+O\left(\varepsilon^{2}\right)} \\
& +\varepsilon\left(\begin{array}{r|r}
-\boldsymbol{B}_{0} \mathrm{R} & -\boldsymbol{B}_{0} \mathrm{~S} \\
\hline \boldsymbol{B}_{0} \mathrm{R} & \boldsymbol{B}_{0} \mathrm{~S}
\end{array}\right)+O\left(\varepsilon^{2}\right),
\end{aligned}
$$

in which $O$ is the zero matrix and $I$ is again the identity. In the first two terms of Eq. (A7) and up to smaller corrections, we recognize the Jacobian $\mathrm{K}_{*}$ of the corresponding model with stochastic switching only, because $\boldsymbol{k}^{\prime}=\boldsymbol{k}+\mathrm{R} \cdot \boldsymbol{B}_{\mathbf{0}}+O(\varepsilon)$. We use these expansions to sample coexistence equilibria of random systems with $\varepsilon \ll 1$ directly, and to determine their stability numerically.

\section{Permanent Coexistence in Eqs. (1)}

To determine whether coexistence is permanent where a coexistence equilibrium is unstable, we perturb the system away from that unstable equilibrium, and evolve it numerically using the stiff solver ode15s of MatLaв (The MathWorks, Inc.). During the numerical solution, we repeatedly test for convergence to a stable equilibrium or a stable limit cycle. In 
particular, limit cycles and their stability are determined using the shooting method described in Ref. [37].

There is one particular numerical difficulty associated with this: the numerical integration generally fails, even at stringent tolerances, if the dynamics approach an attractor partly contained in one of the planes $B_{n}=0$ or $P_{n}=0$. Abundances become arbitrarily small (while remaining nonzero at finite times) on such a trajectory, but such small abundances lack biological meaning. We therefore simply remove such species from the system. In more detail, we declare species $n$ to go extinct at time $t_{n}=\operatorname{argmin}\left\{\max \left\{B_{n}(t), P_{n}(t)\right\}<\epsilon\right\}$, and integrate the system formed by the remaining species for $t>t_{n}$. We choose $\epsilon=10^{-6}$ and find, empirically, that this reduces considerably the proportion of systems for which the numerical integration would otherwise fail.

\section{APPENDIX B: COEXISTENCE WITHOUT PHENOTYPIC VARIATION}

In this Appendix, we rederive the classical results for the stability of two-species Lotka-Volterra competition models [26]. We then obtain the stability conditions for averaged models of this Lotka-Volterra form and that correspond to the simplified models (7), (8), (9). We combine these results with the stability calculations in Appendices C, D, and E to compare these simplified models to their averages.

\section{Coexistence in a two-species Lotka-Volterra model without phenotypic variation}

Coexistence in a two-species Lotka-Volterra competition model without phenotypic variation is a classical problem, discussed, for example, in Ref. [26]. With the aim in mind of comparing this model to the models with phenotypic variation considered in the main text, it will be useful to rederive the results briefly. We consider the competition between species $A^{\prime}, B^{\prime}$ described by the differential equations

$$
\dot{B}^{\prime}=B^{\prime}\left(\omega^{\prime}-\alpha^{\prime} A^{\prime}-\chi^{\prime} B^{\prime}\right), \quad \dot{A}^{\prime}=A^{\prime}\left(\zeta^{\prime}-\eta^{\prime} A^{\prime}-\vartheta^{\prime} B^{\prime}\right),
$$

wherein $\alpha^{\prime}, \zeta^{\prime}, \eta^{\prime}, \vartheta^{\prime}, \chi^{\prime}, \omega^{\prime} \geqslant 0$ are parameters. As in our derivation of Eqs. (6) and (7), we could have scaled time and $A^{\prime}, B^{\prime}$ to set some parameters equal to 1 , but we have not done so in order to be able to relate this model to a model with phenotypic variation in the next subsection [33]. Equations (B1) have a single coexistence state $\mathcal{C}^{\prime}=\left(b^{\prime}, a^{\prime}\right)$, where $b^{\prime}=u^{\prime} / w^{\prime}$ and $a^{\prime}=v^{\prime} / w^{\prime}$, with

$$
\begin{aligned}
& u^{\prime}=\alpha^{\prime} \zeta^{\prime}-\eta^{\prime} \omega^{\prime}, \quad v^{\prime}=\vartheta^{\prime} \omega^{\prime}-\zeta^{\prime} \chi^{\prime}, \\
& w^{\prime}=\alpha^{\prime} \vartheta^{\prime}-\eta^{\prime} \chi^{\prime}=\frac{\chi^{\prime} u^{\prime}+\alpha^{\prime} v^{\prime}}{\omega^{\prime}} .
\end{aligned}
$$

Hence the coexistence state is feasible if and only if $u^{\prime}, v^{\prime}$ have the same sign. The Jacobian matrix evaluated at this steady state is

$$
\left(\begin{array}{cc}
-\chi^{\prime} b^{\prime} & -\alpha^{\prime} b^{\prime} \\
-\vartheta^{\prime} a^{\prime} & -\eta^{\prime} a^{\prime}
\end{array}\right)
$$

Since tr $=-\chi^{\prime} b-\eta^{\prime} a^{\prime}<0$, classical stability results [26] imply that coexistence is stable if and only if $0<\operatorname{det}=-u^{\prime} v^{\prime} / w^{\prime}$. Hence coexistence state is feasible and stable if and only if $u^{\prime}, v^{\prime}<0$, or equivalently, if and only if

$$
\frac{\vartheta^{\prime} \omega^{\prime}}{\chi^{\prime}}<\zeta^{\prime}<\frac{\eta^{\prime} \omega^{\prime}}{\alpha^{\prime}}
$$

Equations (B1) have two additional nonzero steady states, namely $\mathcal{A}^{\prime}=\left(0, \zeta^{\prime} / \eta^{\prime}\right)$ and $\mathcal{B}^{\prime}=\left(\omega^{\prime} / \chi^{\prime}, 0\right)$, at which the Jacobian matrix evaluates to

$$
\left(\begin{array}{cc}
-u^{\prime} / \eta^{\prime} & 0 \\
-\vartheta^{\prime} \zeta^{\prime} / \eta^{\prime} & -\zeta^{\prime}
\end{array}\right) \quad \text { and } \quad\left(\begin{array}{cc}
-\omega^{\prime} & -\alpha^{\prime} \omega^{\prime} / \chi^{\prime} \\
0 & -v^{\prime} / \chi^{\prime}
\end{array}\right)
$$

respectively. Hence $\mathcal{A}^{\prime}$ and $\mathcal{B}^{\prime}$ are stable if $u^{\prime}>0$ and $v^{\prime}>0$, respectively. Comparing these parameter ranges for stability, it follows that either exactly one of $\mathcal{A}^{\prime}, \mathcal{B}^{\prime}, \mathcal{C}^{\prime}$ is stable, or $\mathcal{A}^{\prime}, \mathcal{B}^{\prime}$ are both stable. From arbitrary initial conditions, Eqs. (B1) converge to the stable steady state if is unique; if $\mathcal{A}^{\prime}, \mathcal{B}^{\prime}$ are both stable, a separatrix through $\mathcal{C}^{\prime}$ separates initial conditions converging to $\mathcal{A}^{\prime}$ from those converging to $\mathcal{B}^{\prime}$ [26].

\section{Averages without phenotypic variation of the two-species model (6) and of the simplified models (7), (8), (9)}

We seek to describe the populations $B, P, A$ that evolve according to Eqs. (6) by an averaged model without phenotypic variation and two populations $B^{\prime}$, which corresponds to $B$ and $P$, and $A^{\prime}$, which corresponds to $A$. We have previously introduced this averaging in Ref. [15], and we have motivated it again here, in Section II.

\section{a. Derivation of the averaged model}

A coexistence equilibrium $\mathcal{C}=(b, p, a)$ of Eqs. (6) is consistent with the equilibrium $\mathcal{C}^{\prime}=\left(b^{\prime}, a^{\prime}\right)$ of Eqs. (B1) if and only if the populations, the births, and the competition are equal at equilibrium, i.e. if and only if

$$
\begin{array}{rlrl}
b^{\prime} & =b+p, & a^{\prime} & =a, \\
\omega^{\prime} b^{\prime} & =b+\mu p, \\
\zeta^{\prime} a^{\prime} b^{\prime} & =a(\alpha b+\xi p), & & =\zeta a, \\
\vartheta^{\prime} a^{\prime} b^{\prime} & =a(\vartheta b+\iota p), \\
\chi^{\prime} b^{\prime 2} & =b^{2}+(\kappa+\varpi) b p+\varsigma p^{2}, & \eta^{\prime} a^{\prime 2} & =\eta a^{2} .
\end{array}
$$

These conditions are to Eqs. (6) what conditions (4) are to Eqs. (1). We let $q=p / b$, so that, on introducing $Q=(1+q)^{-1}$, they reduce to

$$
\begin{array}{lll}
\alpha^{\prime}=Q(\alpha+\xi q), & \zeta^{\prime}=\zeta, & \omega^{\prime}=Q(1+\mu q), \\
\vartheta^{\prime}=Q(\vartheta+\iota q), & \eta^{\prime}=\eta, & \chi^{\prime}=Q^{2}\left[1+(\kappa+\varpi) q+\varsigma q^{2}\right] .
\end{array}
$$

Hence Eq. (B4), the stability condition for the averaged model, becomes

$$
\frac{\vartheta+\imath q}{1+(\kappa+\varpi) q+\varsigma q^{2}}<\frac{\zeta}{1+\mu q}<\frac{\eta}{\alpha+\xi q} .
$$




\section{b. Equivalence of the simplified models (7), (8), (9) with $\beta=0$ to a model without phenotypic variation}

If $\xi=\varpi=\varsigma=0$ and $\beta=0$, then $q=\gamma / \tau$, where $\tau=\delta-\mu$. Feasibility requires $\tau>0$. In the cases $\mu=\kappa=0, \iota=\kappa=0$, $\mu=\iota=0$, the interval in Eq. (B8) thus reduces to

$$
\vartheta+\frac{\gamma \iota}{\delta}<\zeta<\frac{\eta}{\alpha}, \vartheta<\frac{\zeta}{1+\mu \gamma / \tau}<\frac{\eta}{\alpha}, \frac{\vartheta}{1+\kappa \gamma / \delta}<\zeta<\frac{\eta}{\alpha},
$$

respectively, which, from the calculations in Appendices $\mathrm{C}$, D, and E, are precisely the stability conditions for Eqs. (7), (8), and (9), respectively, with $\beta=0$. This establishes the one-to-one correspondence between these simplified models and the corresponding averaged models claimed in the main text. We note that, for model (9), this requires an additional condition on the model parameters discussed in Appendix E; this condition follows from the persister scalings (10).

\section{c. Stability of the averages of the simplified models (7), (8), (9)}

$$
\text { for } \beta \neq 0
$$

If now $\xi=\varpi=\varsigma=0$, but $\beta \neq 0$, then $q=(\gamma+\beta a) / \tau$, where, again, $\tau=\delta-\mu>0$. We discuss the three simplified models (7), (8), (9) severally, simplifying the interval (B8) using the explicit expressions for $a$ derived in Appendices $C$, $\mathrm{D}, \mathrm{E}$, respectively.

For model (7), $\mu=\kappa=0$ and $a=a_{ \pm}$, defined in Eq. (C1a) and corresponding to the equilibria $\mathcal{C}_{ \pm}$. The stability conditions (B8) of the averaged model become

$$
U<0, V+\frac{\iota \beta}{\delta} a_{ \pm}<0, \quad \text { with } U=\alpha \zeta-\eta, V=\vartheta-\zeta+\frac{\gamma \iota}{\delta}
$$

as in the definitions (C2a). Since $a_{ \pm}>0$ by feasibility, a necessary condition for stability is $U, V<0$, but this is inconsistent with feasibility of $\mathcal{C}_{+}$(Appendix $\mathrm{C}$ ), which is therefore unstable in the averaged model. Now, using the expression for $a_{-}$in Eq. (C1a), we find

$$
V+\frac{\iota \beta}{\delta} a_{-}=\frac{1}{2 \alpha \delta}\left(Z-\sqrt{Z^{2}+\alpha \delta^{2} U V}\right),
$$

where $Z=\delta(\alpha V-U)+\iota \beta$, and infer that the second condition above is satisfied if $U, V<0$. Since this condition is necessary for stability, $\mathcal{C}_{-}$is stable in the average of model (7) if and only if $U, V<0$.

For model (8), $\iota=\kappa=0$ and $a$ is given in Eqs. (D1). The interval (B8) becomes

$$
\begin{gathered}
U, V<0 \text { if } \vartheta<\frac{\eta}{\alpha} \text { or } \vartheta>\frac{\eta}{\alpha}, \frac{\beta \mu \zeta}{\tau}<\left(1+\frac{\mu \gamma}{\tau}\right)(\alpha \vartheta-\eta), \\
U, V>0 \quad \text { if } \vartheta>\frac{\eta}{\alpha}, \frac{\beta \mu \zeta}{\tau}>\left(1+\frac{\mu \gamma}{\tau}\right)(\alpha \vartheta-\eta),
\end{gathered}
$$

with, now,

$$
U=\alpha \zeta-\eta-\frac{\mu}{\tau}(\gamma \eta+\beta \zeta), \quad V=\vartheta\left(1+\frac{\mu \gamma}{\tau}\right)-\zeta
$$

as defined in Eqs. (D2a), too. From these expressions, it follows that conditions (B12) cannot be satisfied if $\vartheta>\eta / \alpha$. Hence the averaged model corresponding to Eqs. (8) is stable if and only if $\vartheta<\eta / \alpha$ and $U, V<0$.

Finally, for model (9), $\iota=\mu=0$ and $a=a_{ \pm}$, where $a_{ \pm}$are defined in Eq. (E1a) and correspond to the equilibria $\mathcal{C}_{ \pm}$. The stability interval (B8) reduces to

$$
U<0, \quad V<\frac{\beta \kappa \zeta}{\delta} a_{ \pm},
$$

where, now,

$$
U=\alpha \zeta-\eta, \quad V=\vartheta-\left(1+\frac{\gamma \kappa}{\delta}\right) \zeta
$$

as defined in Eqs. (E2a), too. Since $a_{ \pm}>0$ by feasibility, the second condition is clearly satisfied if $V<0$. We may therefore suppose that $V>0$. Now, on letting $Y=\delta(\vartheta U+\eta V) / \zeta+\beta \kappa \zeta$ as in Eqs. (E2b) and using the explicit form of $a_{ \pm}$given in Eq. (E1a), the second condition in Eqs. (B14) becomes

$$
\mp \sqrt{Y^{2}-4 \beta \delta \eta \kappa V}>\frac{2 \delta \eta}{\zeta} V-Y .
$$

We observe that

$$
\frac{2 \delta \eta}{\zeta} V-Y<0 \quad \Longleftrightarrow \quad \beta>\frac{\delta}{\kappa \zeta^{2}}(\eta V-\vartheta U) .
$$

Since we assume that $V>0$, feasibility of $\mathcal{C}_{ \pm}$requires $U<0$ and, from Eq. (E4),

$$
\beta>\frac{\delta}{\kappa \zeta^{2}}(\sqrt{\eta V}+\sqrt{-\vartheta U})^{2}>\frac{\delta}{\kappa \zeta^{2}}(\eta V-\vartheta U),
$$

where the second inequality holds since $(x+y)^{2}>x^{2}+y^{2}$ for $x, y>0$. This shows that inequalities (B17) hold true. Moreover,

$$
\left(Y^{2}-4 \beta \delta \eta \kappa V\right)-\left(\frac{2 \delta \eta}{\zeta} V-Y\right)^{2}=\frac{4 U V \delta^{2} \eta \vartheta}{\zeta}<0
$$

since $U<0, V>0$. It follows from inequalities (B17) and (B19) that condition (B16) holds true. Hence $\mathcal{C}_{ \pm}$is stable in the averaged model if and only if it is feasible.

These results also enable us to compare the simplified models (7), (8), (9) with responsive switching to averaged models with stochastic switching only: the latter have an effective switching rate $\gamma^{\prime}=\gamma+\beta a$. This follows similarly to the correspondence of Eqs. (1) and (2) established in Sec. II and implies that $q^{\prime}=\gamma^{\prime} / \tau=(\gamma+\beta a) / \tau$ as above, i.e. conditions (B9), with $q$ replaced by this $q^{\prime}$, are precisely the conditions that we have just analyzed. This means that the averaged model with stochastic switching only is stable if and only if the averaged model without phenotypic variation is stable. We stress however that there is no reason to expect this "complete" correspondence to hold for the full two-species model (6).

\section{APPENDIX C: FEASIBILITY AND STABILITY OF EQUILIBRIA OF THE SIMPLIFIED MODEL (7)}

In this Appendix, we analyze the feasibility and stability of the equilibria of the simplified model (7). We first give 
the expressions for the coordinates of the coexistence states $\mathcal{C}_{ \pm}=\left(b_{ \pm}, p_{ \pm}, a_{ \pm}\right)$introduced in Eqs. (11b). They are

$$
\begin{aligned}
& a_{ \pm}=\frac{1}{2 \alpha \beta \iota}\left(-Y \pm \sqrt{Y^{2}+4 \alpha \beta \delta \iota V}\right) \\
& b_{ \pm}=\frac{1}{2 \beta \iota}\left(X \mp \sqrt{X^{2}-4 \beta \delta \iota U}\right) \\
& p_{ \pm}=\frac{b_{ \pm}}{\delta}\left(\beta a_{ \pm}+\gamma\right)
\end{aligned}
$$

wherein

$$
\begin{array}{ll}
U=\alpha \zeta-\eta, & V=\vartheta-\zeta+\frac{\gamma \iota}{\delta}, \\
X=\delta(U+\alpha V)+\iota \beta, & Y=\delta(U+\alpha V)-\iota \beta,
\end{array}
$$

so that $X^{2}-4 \beta \delta \iota U=Y^{2}+4 \alpha \beta \delta \iota V$ and $X=Y+2 \beta \iota$. In particular, $X \geqslant Y$. If $\beta=0$ or $\iota=0$, there is but a single coexistence state $\mathcal{C}=(b, p, a)$, with

$$
b=\frac{U}{W}, \quad a=\frac{V}{W}, \quad p=\frac{b}{\delta}(\beta a+\gamma),
$$

wherein $W=U+\alpha V$. From Eqs. (C1c) and (C3), it is immediate that $p_{ \pm}, p>0$ if $b_{ \pm}, b>0$ and $a_{ \pm}, a>0$, and so we need not consider this coordinate to determine feasibility.

\section{Feasibility of the coexistence equilibria}

We now ask whether the coexistence equilibria are feasible. In Table II, we analyze the possible sign combinations of the variables $U, V, X, Y$, defined in Eqs. (C2) and that appear in

TABLE II. Feasibility of the coexistence equilibria $\mathcal{C}_{ \pm}$of Eqs. (7): discussion of the sixteen possible sign combinations of $U, V, X, Y$, defined in Eqs. (C2). For some combinations, the resulting signs of $a_{ \pm}$or $b_{ \pm}$, defined in Eqs. (C1), are given, and the symbol $\mathbb{C}$ is used for

\begin{tabular}{|c|c|c|c|c|c|c|}
\hline$U$ & $V$ & $X$ & $Y$ & $a_{ \pm}$ & $b_{ \pm}$ & \\
\hline+ & + & + & + & \pm & + & only $\mathcal{C}_{+}$is feasible \\
\hline+ & + & + & - & \pm & + & only $\mathcal{C}_{+}$is feasible \\
\hline+ & + & - & + & & & $\#(X<0, Y>0 \Rightarrow X<Y)$ \\
\hline+ & - & + & + & $-/ \mathbb{C}$ & & $\mathcal{C}_{ \pm}$are not feasible \\
\hline- & + & + & + & \pm & $\mp$ & $\mathcal{C}_{ \pm}$are not feasible \\
\hline+ & + & - & - & & & $\#(U, V>0 \Rightarrow X>0)$ \\
\hline+ & - & + & - & $+/ \mathbb{C}$ & $+/ \mathbb{C}$ & $\mathcal{C}_{ \pm}$can both be feasible \\
\hline- & + & + & - & \pm & $\mp$ & $\mathcal{C}_{ \pm}$are not feasible \\
\hline+ & - & - & + & & & $\#(X<0, Y>0 \Rightarrow X<Y)$ \\
\hline- & + & - & + & & & $\#(X<0, Y>0 \Rightarrow X<Y)$ \\
\hline- & - & + & + & & & $\#(U, V<0 \Rightarrow Y<0)$ \\
\hline+ & - & - & - & & $-/ \mathbb{C}$ & $\mathcal{C}_{ \pm}$are not feasible \\
\hline- & + & - & - & \pm & $\mp$ & $\mathcal{C}_{ \pm}$are not feasible \\
\hline- & - & + & - & + & $\mp$ & only $\mathcal{C}_{-}$is feasible \\
\hline- & - & - & + & & & $\#(X<0, Y>0 \Rightarrow X<Y)$ \\
\hline- & - & - & - & + & $\mp$ & only $\mathcal{C}_{-}$is feasible \\
\hline
\end{tabular}
some combinations to indicate that the resulting values of $a_{ \pm}$or $b_{ \pm}$ may have nonzero imaginary part. Some sign combinations, marked \# in the final column, are inconsistent with definitions (C2); for other sign combinations, this column gives the corresponding feasibility results. the coordinates of the equilibria in Eqs. (C1). This shows that only $\mathcal{C}_{+}$is feasible if $U, V>0$, while only $\mathcal{C}_{-}$is feasible if $U, V<0$. Neither coexistence state is feasible if $U<0, V>0$, but it is possible for both coexistence states to be feasible if $U>0, V<0$ provided that $X>0, Y<0$ and that $\mathcal{C}_{ \pm}$are real (Table II). These conditions reduce to

$$
\beta \iota>\delta|U+\alpha V| \text { and }[\delta(U+\alpha V)+\beta \iota]^{2}>4 \beta \delta \iota U .
$$

The second, quadratic condition implies that

$$
\beta \iota<\delta(\sqrt{U}-\sqrt{-\alpha V})^{2} \quad \text { or } \quad \beta \iota>\delta(\sqrt{U}+\sqrt{-\alpha V})^{2} .
$$

Since $(x-y)^{2} \leqslant\left|x^{2}-y^{2}\right|<(x+y)^{2}$ for all $x, y>0$, the first possibility is not consistent with the first condition in Eqs. (C4), while this condition holds in the second case. It follows that both coexistence states are feasible if and only if

$$
U>0, \quad V<0, \quad \text { and } \quad \beta>\beta_{*}=\frac{\delta}{\iota}(\sqrt{U}+\sqrt{-\alpha V})^{2} .
$$

In particular, $U>0$ and $V<0$ requires

$$
\zeta>\max \left\{\vartheta+\frac{\gamma \iota}{\delta}, \frac{\eta}{\alpha}\right\} .
$$

Moreover, letting $W=U+\alpha V$ again, the conditions $X>0$, $Y<0$ imply that $\beta_{*} \iota>\delta|W|$. Combining these results also yields $\beta_{*}>\beta_{*}^{\min }$, with $\beta_{*}^{\text {min }}$ defined in Eq. (12).

This additional region of feasibility does not arise if $\beta=0$ or $\iota=0$. Indeed, it is immediate from Eqs. (C3) that coexistence is feasible in that case if and only if $U, V, W=U+\alpha V$ all have the same sign, and hence if and only if $U, V>0$ or $U, V<0$.

\section{Stability of the coexistence equilibria}

We now turn to the question of stability of the coexistence equilibria. Before discussing the general case $\beta \iota \neq 0$, we discuss two special cases with $\beta \iota=0$. The Jacobian evaluated at a coexistence equilibrium $(b, p, a)$ is

$$
\left(\begin{array}{ccc}
-b-\beta a-\gamma & \delta & -(\alpha+\beta) b \\
\beta a+\gamma & -\delta & \beta b \\
-\vartheta a & -\iota a & -\eta a
\end{array}\right)
$$

a. Stability of the coexistence equilibria if $\beta=0$

In the absence of responsive switching, i.e. if $\beta=0$, and from Eq. (C8), the characteristic polynomial of the Jacobian at $\mathcal{C}=(b, p, a)$, defined by Eqs. (C 3$)$, is

$$
P(\lambda)=W^{2} \lambda^{3}+c_{2} \lambda^{2}+c_{1} \lambda-\delta U V W,
$$

wherein

$$
\begin{aligned}
& c_{1}=\delta U W+(\gamma+\delta) \eta V W-U V w, \\
& c_{2}=[U+\eta V+(\gamma+\delta) W] W,
\end{aligned}
$$


with $w=W-\alpha \gamma \iota / \delta$. The Routh-Hurwitz conditions [26] imply that $\mathcal{C}$ is stable only if $\delta U V W<0$. Recalling that $\mathcal{C}$ is feasible if and only if $U, V, W=U+\alpha V$ all have the same sign, it follows that $\mathcal{C}$ is stable only if $U, V<0$. Moreover, if $U, V<0$ and hence $W<0$, then $c_{2}>0$ and $c_{1} c_{2}>-\delta U V W^{3}$; the second inequality is easily checked by direct multiplication, noting that $w<W<0$. The Routh-Hurwitz conditions then imply that $\mathcal{C}$ is stable if and only if $U, V<0$.

\section{b. Stability of the coexistence equilibria if $\iota=0$}

In the case $\iota=0$, in which the competition dynamics do not involve $P$ directly, we find that the characteristic polynomial of the Jacobian at $\mathcal{C}=(b, p, a)$, defined by Eqs. (C3), still has the form in Eq. (C9), with modified coefficients

$$
\hat{c}_{1}=c_{1}+\beta V(\eta V-\vartheta U), \quad \hat{c}_{2}=c_{2}+\beta V W .
$$

Similarly to the case $\beta=0$ discussed above, $\mathcal{C}$ is stable if and only if $U, V<0$ and $\hat{c}_{1} \hat{c}_{2}>-\delta U V W^{3}$ by the RouthHurwitz conditions [26]. Noting that $U, V, W$ are independent of $\beta$, the latter condition can be written as a quadratic in $\beta$, $d_{2} \beta^{2}+d_{1} \beta+d_{0}>0$, with $d_{0}=c_{1} c_{2}+\delta U V W^{3}>0$ for $U, V<0$ by the above. Moreover, if $U, V<0$ and $\eta V-\vartheta U<0$, all the terms in the definitions (C11) are positive, so $d_{1}, d_{2}>0$ and hence $\hat{c}_{1} \hat{c}_{2}>-\delta U V W^{3}$. If $\eta V>\vartheta U$ however, we find $d_{2}=V^{2} W(\eta V-\vartheta U)<0$ and hence $d_{2} \beta^{2}+d_{1} \beta+d_{0}<0$ for sufficiently large $\beta$. Now $U, V<0 \Longleftrightarrow \vartheta<\zeta<\eta / \alpha$, while $\eta V>\vartheta U$ if and only if $\zeta<2 \eta \vartheta /(\eta+\alpha \vartheta) \equiv \zeta_{*}$, with $\vartheta<\zeta_{*}<\eta / \alpha$ since $\alpha \vartheta-\eta=W<0$. In particular, stable coexistence requires $\vartheta<\eta / \alpha$. If, additionally, $\zeta_{*}<\zeta<\eta / \alpha$, coexistence is stable for all $\beta$, but if $\vartheta<\zeta<\zeta_{*}$, coexistence is only stable for small enough $\beta$.

The condition $d_{2}<0$ is, if $U, V<0$ and hence $W<0$, equivalent with $\eta a<\vartheta b$. This says that destabilization at large $\beta$ requires the death rate of competitors at steady state due to inter-species competition to exceed that due to intraspecies competition.

\section{c. Stability of the coexistence equilibria if $\beta \iota \neq 0$}

Next, we discuss the stability of the two coexistence states $\mathcal{C}_{ \pm}=\left(b_{ \pm}, p_{ \pm}, a_{ \pm}\right)$, defined in Eqs. (C1) for the case $\beta \iota \neq 0$. From Eq. (C8), the characteristic polynomial is

$$
P_{ \pm}(\lambda)=\lambda^{3}+c_{2}^{ \pm} \lambda^{2}+c_{1}^{ \pm} \lambda+c_{0}^{ \pm},
$$

where, in particular and using $W=U+\alpha V$,

$$
c_{0}^{ \pm}=a_{ \pm} b_{ \pm}\left[-\delta W+\beta \iota\left(b_{ \pm}-\alpha a_{ \pm}\right)\right]=\mp a_{ \pm} b_{ \pm} \Delta,
$$

wherein $\Delta^{2}=X^{2}-4 \beta \delta \iota U=Y^{2}+4 \alpha \beta \delta \iota V$ and $\Delta>0$. Hence $c_{0}^{ \pm} \lessgtr 0$, and the Routh-Hurwitz conditions [26] imply in particular that $\mathcal{C}_{+}$is unstable. Further, on comparing to our earlier feasibility results, stable coexistence is only possible at $\mathcal{C}_{-}$and if $U, V<0$ or conditions (C6) are satisfied.

Moreover, $c_{2}^{-}=b_{-}+(\beta+\eta) a_{-}+\gamma+\delta>0$ if $\mathcal{C}_{-}$is feasible, and hence, by the Routh-Hurwitz conditions, stability is in that case equivalent with $c_{1}^{-} c_{2}^{-}>c_{0}^{-}$. In what remains of this Appendix, we analyze this necessary and sufficient condition.

We begin by noting that a region of instability must arise at large $\beta$ provided that $\iota<\vartheta$. Indeed, using MathematicA (Wolfram, Inc.) to simplify complicated expressions, we find that, for $\beta \gg 1$,

$$
c_{0}^{-} \sim-\delta V, c_{1}^{-} \sim \delta\left[1+V\left(\frac{\vartheta}{\iota}-1\right)\right], c_{2}^{-} \sim 1+\gamma+\delta-\frac{V \delta}{\iota},
$$

and hence $c_{1}^{-} c_{2}^{-}-c_{0}^{-} \sim v_{0}+v_{1} V+v_{2} V^{2}$, with, in particular, $v_{0}=\delta(1+\gamma+\delta)>0$ and $v_{2}=-(\vartheta-\iota)(\delta / \iota)^{2}$. Now, from the persister scalings (10), we expect $\iota<\vartheta$, so that $v_{2}<0$. Since $v_{0}>0$, it follows that $\mathcal{C}_{-}$is unstable at large $\beta$ if and only if $|V|$ is large enough. This condition is different from the one that we obtained above when discussing $\iota=0$, for which we showed that instability must occur at large $\beta$ if $|V|$ is small enough. This emphasizes that the limit $\iota=0$ is singular.

This asymptotic condition for instability at large $\beta$ is independent of $\alpha, \eta$, and thus of $U$. Hence instability may, but need not occur in the region $U, V<0$. If such a region of instability exists then, because $\mathcal{A}, \mathcal{B}$ are unstable if $U, V<0$, all steady states are in fact unstable in this region. This discussion also shows that instability must occur at large $\beta$ under the conditions described by Eqs. (C6).

Coexistence is stable, however, for small $\beta$ if $U, V<0$ and hence $W<0$. Indeed, a straightforward calculation shows that $\mathcal{C}_{-} \rightarrow \mathcal{C}$ as $\beta \rightarrow 0$ at fixed $\iota \neq 0$, with $\mathcal{C}$ defined as in Eqs. (C3). Since $\mathcal{C}$ is stable for $U, V<0$, so is $\mathcal{C}_{-}$for sufficiently small $\beta$ by continuity.

Moreover, $\mathcal{C}_{-}$is stable for $V<0$ and $|V|$ sufficiently small. Indeed, notice that $\Delta=|\beta \iota-\delta U|+O(V)$. Now, if $U<0$, $\beta \iota-\delta U>0$. If $U>0$, feasibility requires $\beta \iota>\delta U+O(V)$ from Eqs. (C6), and so $\Delta=\beta \iota-\delta U+O(V)$ in either case. Direct computation then yields $c_{1}^{-} c_{2}^{-}-c_{0}^{-}=v_{0}+O(V)>0$, so $\mathcal{C}_{-}$is stable, as claimed.

The question whether $\mathcal{C}_{-}$is stable more generally under the conditions in Eqs. (C6) requires somewhat more effort. First, we discuss the limit in which $\beta, \zeta \gg 1$, considering all other parameters to be $O(1)$ quantities. Moreover, we assume that $r=\beta \iota / \alpha \delta \zeta=O(1)$. With these scalings, $U>0$ and $V<0$, so the feasibility conditions (C6) reduce to $r>4$. We then find

$$
\begin{aligned}
& c_{0}^{-} \sim \delta \zeta \sqrt{1-4 r^{-1}}, \quad c_{2}^{-} \sim \frac{\beta}{2 \alpha}\left(1-\sqrt{1-4 r^{-1}}\right), \\
& c_{1}^{-} \sim \frac{\beta \eta}{2 \alpha^{2}}\left(1-\sqrt{1-4 r^{-1}}\right)-\frac{\delta \zeta}{\alpha \iota}[\alpha(\vartheta-\iota)+\eta],
\end{aligned}
$$

and hence $c_{1}^{-} c_{2}^{-}-c_{0}^{-}>0$ for sufficiently large $\beta$ if and only if $c_{1}^{-}>0$, which is if and only if

$$
r\left(1-\sqrt{1-4 r^{-1}}\right)>2 s, \quad \text { where } s=1+\alpha(\vartheta-\iota) / \eta
$$

or, equivalently, if and only if

$$
4<r<r_{*}=\frac{s^{2}}{s-1} \quad \text { and } \quad 1<s<2 .
$$


Assuming that $\vartheta>\iota$ as discussed above, $s>1$. We conclude that there exists a region of parameter space in which coexistence is stable for $\beta, \zeta \gg 1$ under the condition in Eqs. (C6) if and only if $\alpha(\vartheta-\imath)<\eta$. We also note that $\alpha(\vartheta-\imath)<\eta$ is implied by the condition $W<0$.

Finally, we consider stability near the feasibility boundary $\beta=\beta_{*}$ defined in Eqs. (C6). By continuity, stability near this boundary follows from stability at the boundary. Now, by definition $\Delta=0$ and hence, from Eq. (C13), $c_{0}^{-}=0$ at this boundary, so stability there is equivalent with $c_{1}^{-}>0$ since $c_{2}^{-}>0$. Using $\Delta=0$, direct calculation shows that

$$
\begin{aligned}
(2 \alpha \beta \iota)^{2} c_{1}^{-}=[ & -\alpha(\vartheta-\iota)] \beta(\beta \iota-\delta W)(\beta \iota+\delta W+2 \delta \eta) \\
& +\alpha(\eta-\alpha \vartheta)\left[(\beta \iota)^{2}-(\delta W)^{2}\right] \\
& +2 \alpha^{2} \delta \beta \iota(\beta \iota+\delta W) \\
=[\eta & -\alpha(\vartheta-\iota)](\beta \iota-\delta W)[\beta(\beta \iota+\delta W+2 \delta \eta) \\
& +\alpha(\beta \iota+\delta W)] \\
& +\alpha^{2}(\beta \iota+\delta W)[(2 \delta-\iota) \beta \iota+\delta \iota W] . \quad(\mathrm{C} 17 \mathrm{~b})
\end{aligned}
$$

The discussion around Eqs. (C6) implies that $\beta \iota>|\delta W|$. Hence $c_{1}^{-}>0$ if $\eta>\alpha \vartheta$ from Eq. (C17a). If $\iota<\delta$, then $(2 \delta-\iota) \beta \iota+\delta \iota W>\iota(\beta \iota+\delta W)>0$, so Eq. (C17b) shows that $c_{1}^{-}>0$ continues to hold if $\alpha(\vartheta-\iota)<\eta<\alpha \vartheta$ provided that $\iota<\delta$. We expect this to be true from the persister scalings (10). If $\iota>\delta$ however, parameter values such that $c_{1}^{-}<0$ do exist. All of this shows that coexistence is stable if $\eta>\alpha(\vartheta-\imath)$, provided that $\iota<\delta$. Moreover, rearranging Eqs. (C17a) or (C17b) yields

$$
\begin{gathered}
(2 \alpha \beta \iota)^{2} c_{1}^{-}=\iota^{2}[\eta-\alpha(\vartheta-\iota)] \beta^{3}+d \beta^{2}+\beta \delta W\{\alpha \delta \iota \\
-[\eta-\alpha(\vartheta-\iota)](\alpha \delta \vartheta+\delta \eta+\alpha \gamma \iota)\} \\
+\alpha \delta^{2} W^{2}(\alpha \vartheta-\eta), \quad(\mathrm{C} 17
\end{gathered}
$$

in which the value of the coefficient $d$ is of no consequence. Hence, if $\eta<\alpha(\vartheta-\imath)$, then $c_{1}^{-}<0$ and coexistence is unstable for sufficiently large $\beta$; we obtained the same result above. Here, we note additionally that, if $\eta<\alpha(\vartheta-\imath)$, then $W>0$, and so the linear and constant terms of the cubic (C17c) are positive, while its cubic term is negative, so it has exactly one positive real root. Since $\mathcal{C}_{-}$is stable for $|V|$ sufficiently small, this root of $c_{1}^{-}$corresponds to a point on the feasibility boundary $\Delta=0$. Thus the boundary of the unstable region intersects the feasibility boundary as shown in Fig. 5(b).

While even combining all of these results does not give a complete description of the region of parameter space in which $\mathcal{C}_{-}$is feasible and stable, this combination yields the qualitative stability diagrams shown in Fig. 5.

\section{Coexistence beyond coexistence equilibria}

We now extend these results on the coexistence of the two species described by Eqs. (7) beyond coexistence at steady state. We begin by analyzing the stability of the trivial equilibria and the dynamics of Eqs. (7) near the boundaries $B P A=0$. These results will enable us to discuss, partially, the alternative outcomes of extinction of one species and permanent coexistence [31] of the two species.

\section{a. Stability of the trivial steady states}

First, we determine the stability of the trivial steady states $\mathcal{O}, \mathcal{A}, \mathcal{B}$ defined in Eqs. (11a), and which are feasible for all parameter values. The Jacobian of Eqs. (7) evaluated at $\mathcal{O}$ is

$$
\left(\begin{array}{ccc}
1-\gamma & \delta & 0 \\
\gamma & -\delta & 0 \\
0 & 0 & \zeta
\end{array}\right)
$$

In particular, this Jacobian has an eigenvalue $\zeta>0$, so $\mathcal{O}$ is always unstable, with small perturbations expelled from the plane $A=0$.

The stability of the remaining steady states depends on the values of $U, V$. The Jacobian at $\mathcal{A}$ is

$$
\left(\begin{array}{ccc}
-(\gamma+\beta \zeta / \eta)-U / \eta & \delta & 0 \\
\gamma+\beta \zeta / \eta & -\delta & 0 \\
& & -\zeta
\end{array}\right)
$$

in which the entries left blank clearly do not affect stability. Similarly, the Jacobian at $\mathcal{B}$ is

$$
\left(\begin{array}{ccc}
-1-\gamma & \delta & \\
\gamma & -\delta & \\
0 & 0 & -V
\end{array}\right)
$$

Direct computation of eigenvalues shows that $\mathcal{A}$ is stable if and only if $U>0$, while $\mathcal{B}$ is stable if and only if $V>0$. In more detail, computation of eigenvalues shows that $\mathcal{B}$ is an attractor in the plane $A=0$, but repels orbits out of that plane if and only if $V<0$.

It follows that, if $U>0$ or $V>0$, then there exist (feasible) initial conditions with which Eqs. (7) converge to $\mathcal{A}$ or $\mathcal{B}$, and hence lead to extinction of one species, and so permanent coexistence of the two species is not possible in general.

\section{b. Permanent Coexistence}

The above shows that permanent coexistence irrespective of the initial conditions is only possible if $U, V<0$ and hence $\mathcal{A}$ and $\mathcal{B}$ are unstable, assumed henceforth.

From the conditions obtained in Appendix A, the dynamics of Eqs. (7) are bounded. Hence extinction of one species requires the dynamics of Eqs. (7) to converge to a (stable) limit set $\mathcal{L}$ that intersects the boundary $B P A=0$. We claim such a limit set $\mathcal{L}$ cannot exist if $\mathcal{A}$ and $\mathcal{B}$ are unstable [38].

To prove this claim, we begin by noting that, from Eqs. (7), $B=0 \Longrightarrow \dot{B}>0$ and $P=0 \Longrightarrow \dot{P}>0$ unless $B=P=0$; also, if $A=0$, then $\dot{A}=0$. Hence $\mathcal{L} \cap\{B P A=0\}$ lies in the union of the plane $\Pi=\{A=0 ; B, P>0\}$ and the ray $\{B=P=0 ; A \geqslant 0\}$ [Fig. 13(a)].

Next, we observe that the dynamics of Eqs. (7) do not allow $\mathcal{L} \subset \Pi$ : if this were the case, the Poincaré-Bendixson theorem [39] would imply that $\mathcal{L}$ is (1) a fixed point, (2) a limit cycle, or (3) a connection of equilibria. However, (1) is not possible because $\mathcal{O}$ and $\mathcal{B}$ are both unstable, the latter by assumption; (2) is not possible because a limit cycle would necessarily contain the only interior equilibrium, $\mathcal{B}$, 


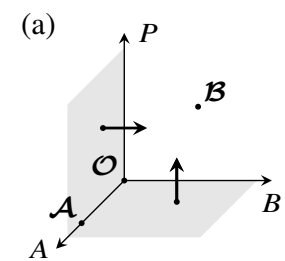

(d)
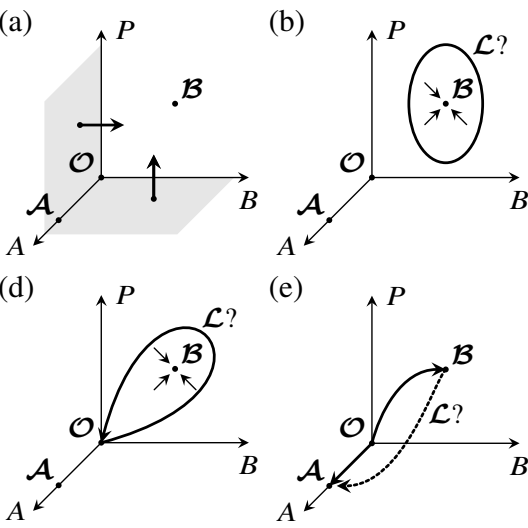

(e)
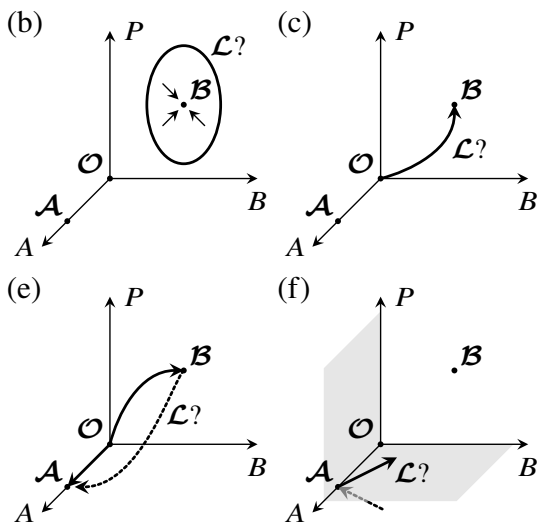

FIG. 13. Permanent coexistence of the two species described by Eqs. (7) if $\mathcal{A}, \mathcal{B}$ are unstable. (a) Orbits of Eqs. (7) are expelled from $B=0$ and $P=0$. (b) Non-existence of a (stable) limit cycle $\mathcal{L}$ in $\Pi=\{A=0 ; B, P>0\}$. (c) Non-existence of a connection $\mathcal{L}$ containing $\mathcal{B}$ in $\Pi$. (d) Non-existence of a homoclinic connection of $\mathcal{O}$ in $\Pi$. (e) Non-existence of a connection containing $\mathcal{O} \rightarrow \mathcal{B}$. (f) Non-existence of a connection containing $\mathcal{A}$, because one of the connections of $\mathcal{A}$ is not feasible.

which is impossible because the latter is, as we have noted below Eq. (C18c), stable in the plane $A=0$ [Fig. 13(b)]; (3) is not possible, because this connection would either contain $\mathcal{B}$ [Fig. 13(c)], or be a homoclinic connection of $\mathcal{O}$ circling $\mathcal{B}$ [Fig. 13(d)], both of which are again impossible because $\mathcal{B}$ is stable in the plane $A=0$. That no limit cycle exists can also be established (less geometrically) using Dulac's criterion [39].

Extending these arguments, $\mathcal{L}$ cannot in fact intersect $\Pi$, for if it did, then it would contain a connection $\mathcal{O} \rightarrow \mathcal{B}$, which is impossible because, as noted above, the directions transverse to $\Pi$ are unstable for both $\mathcal{O}$ and $\mathcal{B}$ [Fig. 13(e)].

Hence $\mathcal{L}$ must intersect $\{B=P=0 ; A>0\}$, so must contain $\mathcal{A}$ and and two of its connections. Since it cannot contain the connection $\mathcal{O} \rightarrow \mathcal{A}$ by the above, it must contain the two other connections of $\mathcal{A}$. However, direct computation of the eigenvectors of the corresponding Jacobian in Eq. (C18b) shows that one of these is not feasible [Fig. 13(f)]. This is the final contradiction showing that $\mathcal{L}$ cannot intersect $B P A=0$.

This argument shows that both species coexist permanently if $\mathcal{A}, \mathcal{B}$ are unstable. The implications of this result are discussed in the main text.

\section{APPENDIX D: FEASIBILITY AND STABILITY OF EQUILIBRIA OF THE SIMPLIFIED MODEL (8)}

In this Appendix, we analyze the feasibility and stability of the equilibria of the simplified model (8).

\section{Equilibria and their feasibility}

Equations (8) have three trivial steady states $\mathcal{O}, \mathcal{A}, \mathcal{B}$ defined by Eqs. (13a). Clearly, $\mathcal{O}$ and $\mathcal{A}$ are feasible, but, letting $\tau=\delta-\mu, \mathcal{B}$ is feasible if and only if $\tau>0$. Moreover, Eqs. (8) have a single coexistence state $\mathcal{C}=(b, p, a)$, with

$$
b=\frac{U}{W}, \quad a=\frac{V}{W}, \quad p=\frac{b}{\tau}(\beta a+\gamma),
$$

wherein

$$
\begin{aligned}
& U=\alpha \zeta-\eta-\frac{\mu}{\tau}(\gamma \eta+\beta \zeta), \quad V=\vartheta\left(1+\frac{\mu \gamma}{\tau}\right)-\zeta, \\
& W=\alpha \vartheta-\eta-\frac{\mu \beta \vartheta}{\tau}=\frac{\vartheta U+\eta V}{\zeta} .
\end{aligned}
$$

If $a, b>0$, then $p>0$ if and only if $\tau>0$, assumed henceforth. The coexistence state $\mathcal{C}$ is then feasible if and only if $U, V, W$ have the same sign, which is, from Eq. (D2b), if and only if $U, V$ have the same sign. We also note that the results of Appendix A show that $\tau>0$ is a sufficient condition for the dynamics of model (8) to be bounded.

\section{Stability of the coexistence equilibria}

Next, we analyze the stability of the coexistence equilibrium. As announced in the main text, we will assume that $\mu>0$; the case $\mu=0$ is equivalent to the case $\iota=0$ discussed in Appendix $\mathrm{C}$. We will first discuss the case $\beta=0$ before analyzing $\beta \neq 0$. The Jacobian evaluated at $\mathcal{C}$ is

$$
\left(\begin{array}{ccc}
-b-(\beta a+\gamma) \delta / \tau & \delta & -(\alpha+\beta) b \\
\beta a+\gamma & -\tau & \beta b \\
-\vartheta a & 0 & -\eta a
\end{array}\right)
$$

$$
\text { a. Stability of coexistence if } \beta=0
$$

If $\beta=0$, i.e. in the absence of responsive switching, the Jacobian (D3) has characteristic polynomial

$$
P(\lambda)=W^{2} \lambda^{3}+c_{2} \lambda^{2}+c_{1} \lambda-\tau U V W,
$$

wherein

$$
\begin{aligned}
& c_{1}=[\tau U+\eta V(\gamma \delta / \tau+\tau)-U V] W, \\
& c_{2}=[U+\eta V+(\gamma \delta / \tau+\tau) W] W .
\end{aligned}
$$

The Routh-Hurwitz conditions [26] imply that coexistence is stable only if $\tau U V W<0$, i.e. only if $U, V<0$ using the feasibility conditions. Conversely, if $U, V<0$ and hence $W<0$, then $c_{2}>0$ and $c_{1} c_{2}>-\tau U V W^{3}$, of which the second inequality is easily checked by direct multiplication. The Routh-Hurwitz conditions thus imply that coexistence is stable if and only if $U, V<0$.

\section{b. Stability of coexistence if $\beta \neq 0$}

If $\beta \neq 0$, the characteristic polynomial of the Jacobian (D3) still has the form in Eq. (D4), with modified coefficients

$$
\hat{c}_{1}=c_{1}+\beta V(\eta V-\vartheta U) \delta / \tau, \quad \hat{c}_{2}=c_{2}+\beta \delta V W / \tau .
$$


As in the above analysis of the case $\beta=0$, the Routh-Hurwitz conditions [26] imply that coexistence is feasible and stable if and only if $U, V<0$ and $\hat{c}_{1} \hat{c}_{2}>-\tau U V W^{3}$.

The latter condition is not implied by $U, V<0$, although coexistence is stable if $|U|$ or $|V|$ is sufficiently small away from the singular point $U=V=0$. We prove this claim by expanding the final Routh-Hurwitz condition in $U$ and $V$, assuming all other parameters to be $O(1)$ quantities. Using Mathematica to handle complicated expressions, we obtain

$$
\begin{aligned}
\hat{c}_{1} \hat{c}_{2}+\tau U V W^{3} & =\frac{V^{4} \eta^{2} u(u+\zeta \eta \tau)}{\zeta^{3} \tau^{2}}+O(U), \\
& =\frac{U^{4} \vartheta^{2}}{\zeta^{3}}\left(\gamma \delta \vartheta+\zeta \tau+\vartheta \tau^{2}\right)+O(V),
\end{aligned}
$$

wherein $u=\beta \delta \zeta+\gamma \delta \eta+\eta \tau^{2}$, which proves our claim. A region of instability must however arise for $\beta, \zeta \gg 1$. This follows from the expansion

$$
\hat{c}_{1} \hat{c}_{2}+\tau U V W^{3}=-\left[\frac{\vartheta^{2} \mu^{2}}{\tau^{3}}(\delta+\mu)\right] \zeta^{3} \beta^{4}+O\left(\zeta^{2} \beta^{4}, \zeta^{3} \beta^{3}\right)
$$

Within this region of instability, $U, V<0$, and so $\mathcal{A}, \mathcal{B}$ are unstable there, too, whence all steady states of Eqs. (8) are unstable in that region.

Moreover, Eqs. (D6) show that $\hat{c}_{1} \rightarrow c_{1}$ and $\hat{c}_{2} \rightarrow c_{2}$ as $\beta \rightarrow 0$. Since $c_{1} c_{2}+\tau U V W^{3}>0$, it follows that, for sufficiently small $\beta, \hat{c}_{1} \hat{c}_{2}+\tau U V W^{3}>0$, too. In other words, for small $\beta, \mathcal{C}$ is stable if and only if $U, V<0$.

If $\vartheta<\eta / \alpha$, then $U, V<0$ is possible if $\beta=0$, but, if $\vartheta>\eta / \alpha$, this requires $\zeta>\vartheta(1+\mu \gamma / \tau)$ and

$$
\beta>\beta_{*}=\frac{\tau}{\mu} \alpha-\left(\gamma+\frac{\tau}{\mu}\right) \frac{\eta}{\zeta},
$$

using Eqs. (D2a). In particular, this implies the lower bound $\beta_{*}>\beta_{*}^{\min }=\tau(\alpha \vartheta-\eta) / \mu \vartheta$ in Eq. (14).

We are left to discuss the stability of $\mathcal{C}$ near the singular point $U=V=0$ for $\vartheta>\eta / \alpha$. This corresponds, in the $(\zeta, \beta)$ plane, to $\zeta=\zeta_{*}^{\min }=\vartheta(1+\mu \gamma / \tau)$ and $\beta=\beta_{*}^{\min }$. In particular, it lies in the first $(\zeta, \beta)$ quadrant if and only if $\vartheta>\eta / \alpha$. Near this point, we write

$$
\frac{\zeta}{\zeta_{*}^{\min }}=1+\bar{\zeta}, \quad \frac{\beta}{\beta_{*}^{\min }}=1+\bar{\beta},
$$

with $\bar{\zeta}, \bar{\beta}>0$. Inserting these definitions into Eqs. (D2a) shows that the domain $U, V<0$ in which $\mathcal{C}$ can be stable corresponds, at leading order, to $\bar{\beta}>\eta \bar{\zeta} / w$, with $w=\alpha \vartheta-\eta>0$. Similarly, from Eqs. (D2), (D5), and (D7), the stability boundary $\hat{c}_{1} \hat{c}_{2}+\tau U V W^{3}=0$ corresponds to the straight lines $\bar{\beta}=0$, $\bar{\beta}=g_{0} \bar{\zeta}$, where

$$
g_{0}=\frac{\eta}{w} \frac{\gamma \mu+\tau}{(\gamma \mu+\tau)+\left(\gamma \delta+\tau^{2}\right)}\left(1-\vartheta-\frac{\delta w}{\mu \eta}\right),
$$

and additionally, if the roots $g_{ \pm}$of the quadratic

$$
\begin{gathered}
\left(\mu \tau^{2} w\right) g^{2}-\left[\mu \tau^{2} \eta+w \delta \vartheta(\gamma \mu+\tau)-\mu \eta \vartheta\left(\delta \gamma+\tau^{2}\right)\right] g \\
+2 \delta \eta \vartheta(\gamma \mu+\tau)=0
\end{gathered}
$$

are real, to the straight lines $\bar{\beta}=g_{+} \bar{\zeta}, \bar{\beta}=g_{-} \bar{\zeta}$. Clearly, $\bar{\beta}=0$ is outside $\bar{\beta}>\eta \bar{\zeta} / w$. It is also easy to see that $g_{0}<\eta / w$, whence so is $\bar{\beta}=g_{0} \bar{\zeta}$. A region of instability near the singular point $U=V=0$ can therefore arise only if $g_{ \pm}$are real, and $g_{+}>\eta / w$ or $g_{-}>\eta / w$. Now $g_{ \pm}$are real if and only if

$$
\begin{aligned}
D \equiv & {\left[\mu \tau^{2} \eta+w \delta \vartheta(\gamma \mu+\tau)-\mu \eta \vartheta\left(\delta \gamma+\tau^{2}\right)\right]^{2} } \\
& -8 \mu \tau^{2} w \delta \eta \vartheta(\gamma \mu+\tau) \geqslant 0
\end{aligned}
$$

while $g_{ \pm}>\eta / w$ if and only if, additionally,

$$
w \delta \vartheta(\gamma \mu+\tau)-\mu \eta \vartheta\left(\delta \gamma+\tau^{2}\right)-\mu \tau^{2} \eta \geqslant \mp \sqrt{D} .
$$

Direct computation shows that

$$
\begin{aligned}
& {\left[w \delta \vartheta(\gamma \mu+\tau)-\mu \eta \vartheta\left(\delta \gamma+\tau^{2}\right)-\mu \tau^{2} \eta\right]^{2}-D} \\
& \quad=4 \eta \vartheta \mu \tau^{2}\left[w \delta(\gamma \mu+\tau)+\eta \mu\left(\delta \gamma+\tau^{2}\right)\right] \geqslant 0,
\end{aligned}
$$

and so inequality (D13a) holds if and only if

$$
w \delta \vartheta(\gamma \mu+\tau) \geqslant \mu \eta\left[\vartheta\left(\delta \gamma+\tau^{2}\right)+\tau^{2}\right] .
$$

This implies in particular that $g_{+}>\eta / w \Longleftrightarrow g_{-}>\eta / w$. We could have obtained this result directly: if only one of $\bar{\beta}=g_{+} \bar{\zeta}$, $\bar{\beta}=g_{-} \bar{\zeta}$ intersected $\bar{\beta}>\eta \bar{\zeta} / w, \mathcal{C}$ would be unstable at one of $U=0, V=0$ for $|V|$ or $|U|$ sufficiently small, but this would contradict Eq. (D7a) or Eq. (D7b). Equations (D12) and (D13c) give the necessary and sufficient conditions for a region of instability to arise near the singular point $U=V=0$.

We obtain the qualitative stability diagrams in $(\zeta, \beta)$ space shown in Fig. 6 by combining these stability results.

\section{Stability of the trivial steady states and permanence}

As in Appendix C, we discuss the stability of the trivial steady states to obtain conditions for permanent coexistence. Again, $\mathcal{O}$ is clearly unstable, because the Jacobian of Eqs. (8) at this equilibrium is

$$
\left(\begin{array}{ccc}
1-\gamma & \delta & 0 \\
\gamma & -\tau & 0 \\
0 & 0 & \zeta
\end{array}\right)
$$

The Jacobian at $\mathcal{A}$ is

$$
\left(\begin{array}{ccc}
-U / \eta-(\gamma+\beta \zeta / \eta) \delta / \tau & \delta & 0 \\
\gamma+\beta \zeta / \eta & -\tau & 0 \\
& & -\zeta
\end{array}\right) .
$$

Again, entries that do not affect stability have been left blank. Similarly, the Jacobian evaluated at $\mathcal{B}$ is

$$
\left(\begin{array}{ccc}
-1-\gamma(\delta+\mu) / \tau & \delta & \\
\gamma & -\tau & \\
0 & 0 & -V
\end{array}\right) .
$$

On computing the eigenvalues of these Jacobians and since we assume that $\tau>0$, we conclude, again, that $\mathcal{A}$ is stable if and only if $U>0$, while $\mathcal{B}$ is stable if and only if $V>0$. We note 
from Eqs. (D2a) that $U>0$ is not possible and hence that $\mathcal{A}$ is unstable if $\beta>\alpha(\delta / \mu-1)$.

The geometric properties of these Jacobians are identical to those of the corresponding equilibria of Eqs. (7) analyzed in Appendix C; this follows from direct computation of their eigenvectors. Hence the argument deployed there to establish permanence of coexistence carries over to model (8): if $\mathcal{A}$ and $\mathcal{B}$ are both unstable, i.e. if $U, V<0$, then the two species coexist permanently.

\section{APPENDIX E: FEASIBILITY AND STABILITY OF EQUILIBRIA OF THE SIMPLIFIED MODEL (9)}

In this Appendix, we analyze the feasibility and stability of the equilibria of the simplified model (9). The trivial steady states $\mathcal{O}, \mathcal{A}, \mathcal{B}$ defined in Eqs. (15a) are clearly feasible. The coexistence states are $\mathcal{C}_{ \pm}=\left(b_{ \pm}, p_{ \pm}, a_{ \pm}\right)$, as defined in Eqs. (15b), with

$$
\begin{aligned}
& a_{ \pm}=\frac{1}{2 \beta \eta \kappa}\left(Y \mp \sqrt{Y^{2}-4 \beta \delta \eta \kappa V}\right), \\
& b_{ \pm}=\frac{1}{2 \beta \vartheta \kappa}\left(-X \pm \sqrt{X^{2}+4 \beta \delta \vartheta \kappa U}\right), \\
& p_{ \pm}=\frac{b_{ \pm}}{\delta}\left(\beta a_{ \pm}+\gamma\right),
\end{aligned}
$$

wherein

$$
\begin{aligned}
U & =\alpha \zeta-\eta, & V & =\vartheta-\left(1+\frac{\gamma \kappa}{\delta}\right) \zeta, \\
X & =\frac{\delta}{\zeta}(\vartheta U+\eta V)-\beta \kappa \zeta, & Y & =\frac{\delta}{\zeta}(\vartheta U+\eta V)+\beta \kappa \zeta,
\end{aligned}
$$

so that $X^{2}+4 \beta \delta \vartheta \kappa U=Y^{2}-4 \beta \delta \eta \kappa V$ and $Y=X+2 \beta \kappa \zeta$. In particular, $X \leqslant Y$. Similarly to the analysis of model (7) in Appendix $\mathrm{C}$, there is but a single coexistence state $\mathcal{C}=(b, p, a)$ if $\beta=0$ or $\kappa=0$, where

$$
b=\frac{U}{W}, \quad a=\frac{V}{W}, \quad p=\frac{b}{\delta}(\beta a+\gamma),
$$

with $W=(\vartheta U+\eta V) / \zeta$. From Eqs. (E1c) and (E3), it is again immediate that $p_{ \pm}, p>0$ if $b_{ \pm}, b>0$ and $a_{ \pm}, a>0$, and so, again, we need not consider this coordinate to determine feasibility.

\section{Feasibility of the coexistence equilibria}

We now have to ask whether the coexistence equilibria $\mathcal{C}_{ \pm}$ and $\mathcal{C}$ are feasible. The calculations are similar to those in Appendix C. In Table III, we analyze the possible sign combinations of the variables $U, V, X, Y$, defined in Eqs. (E2), that appear in the coordinates of the equilibria in Eqs. (E1). We infer that only $\mathcal{C}_{+}$is feasible if $U, V>0$, while only $\mathcal{C}_{-}$is feasible if $U, V<0$. Neither coexistence state is feasible if $U>0$, $V<0$, but both coexistence states are feasible if $U<0, V>0$ provided that $X<0, Y>0$ and that $\mathcal{C}_{ \pm}$are real (Table III).
TABLE III. Feasibility of the coexistence equilibria $\mathcal{C}_{ \pm}$of Eqs. (9): discussion of the sixteen possible sign combinations of $U, V, X, Y$, defined in Eqs. (E2). For some combinations, the resulting signs of $a_{ \pm}$or $b_{ \pm}$, defined in Eqs. (E1), are given, and the symbol $\mathbb{C}$ is used for

\begin{tabular}{|c|c|c|c|c|c|c|}
\hline$U$ & $V$ & $X$ & $Y$ & $a_{ \pm}$ & $b_{ \pm}$ & \\
\hline+ & + & + & + & + & \pm & only $\mathcal{C}_{+}$is feasible \\
\hline+ & + & + & - & & & $\#(X>0, Y<0 \Rightarrow X>Y)$ \\
\hline+ & + & - & + & + & \pm & only $\mathcal{C}_{+}$is feasible \\
\hline+ & - & + & + & $\mp$ & \pm & $\mathcal{C}_{ \pm}$are not feasible \\
\hline- & + & + & + & & $-/ \mathbb{C}$ & $\mathcal{C}_{ \pm}$are not feasible \\
\hline+ & + & - & - & & & $\#(U, V>0 \Rightarrow Y>0)$ \\
\hline+ & - & + & - & & & $\#(X>0, Y<0 \Rightarrow X>Y)$ \\
\hline- & + & + & - & & & $\#(X>0, Y<0 \Rightarrow X>Y)$ \\
\hline+ & - & - & + & $\mp$ & \pm & $\mathcal{C}_{ \pm}$are not feasible \\
\hline- & + & - & + & $+/ \mathbb{C}$ & $+/ \mathbb{C}$ & $\mathcal{C}_{ \pm}$can both be feasible \\
\hline- & - & + & + & & & $\#(U, V<0 \Rightarrow X<0)$ \\
\hline+ & - & - & - & $\mp$ & \pm & $\mathcal{C}_{ \pm}$are not feasible \\
\hline- & + & - & - & $-/ \mathbb{C}$ & & $\mathcal{C}_{ \pm}$are not feasible \\
\hline- & - & + & - & & & $\#(U, V<0 \Rightarrow X<0)$ \\
\hline- & - & - & + & $\mp$ & + & only $\mathcal{C}_{-}$is feasible \\
\hline- & - & - & - & $\mp$ & + & only $\mathcal{C}_{-}$is feasible \\
\hline
\end{tabular}
some combinations to indicate that the resulting values of $a_{ \pm}$or $b_{ \pm}$ may have nonzero imaginary part. Some sign combinations, marked \# in the final column, are inconsistent with definitions (E2); for other sign combinations, this column gives the corresponding feasibility results.

Similarly to Appendix C, we conclude that both coexistence states are feasible if and only if

$$
U<0, \quad V>0, \quad \text { and } \quad \beta>\beta_{*}=\frac{\delta}{\kappa \zeta^{2}}(\sqrt{-\vartheta U}+\sqrt{\eta V})^{2} \text {. }
$$

In particular, $U<0$ and $V>0$ requires

$$
\zeta<\min \left\{\vartheta\left(1+\frac{\gamma \kappa}{\delta}\right)^{-1}, \frac{\eta}{\alpha}\right\} .
$$

Moreover, letting $W=(\vartheta U+\eta V) / \zeta$ again, the conditions $X<0, Y>0$ imply that $\beta_{*}>\delta|W| / \kappa \zeta$. These results also yield the lower bound on $\beta_{*}$ in Eq. (16).

This additional region of feasibility does not arise if $\beta=0$ or $\kappa=0$. Indeed, similarly to Appendix C, Eqs. (E3) show that coexistence is feasible in that case if and only if $U, V$, $W=(\vartheta U+\eta V) / \zeta$ all have the same sign, and hence if and only if $U, V>0$ or $U, V<0$.

\section{Stability of the coexistence equilibria}

Next, we analyze the stability of the coexistence equilibria. We can assume that $\kappa>0$, since the case $\kappa=0$ is equivalent to the case $\iota=0$ in Appendix C. In now familiar fashion, we discuss the cases $\beta=0$ and $\beta \neq 0$ separately, but note that, in both cases, the Jacobian evaluated at a coexistence equilibrium $(b, p, a)$ is

$$
\left(\begin{array}{ccc}
-b-(\beta a+\gamma) & \delta-\kappa b & -(\alpha+\beta) b \\
\beta a+\gamma & -\delta & \beta b \\
-\vartheta a & 0 & -\eta a
\end{array}\right)
$$




\section{a. Stability of coexistence if $\beta=0$}

If $\beta=0$, the characteristic polynomial of Eq. (E6) evaluated at $\mathcal{C}=(b, p, a)$ defined in Eqs. (E3) is

$$
P(\lambda)=W^{2} \lambda^{3}+c_{2} \lambda^{2}+c_{1} \lambda-\delta U V W,
$$

where

$$
\begin{aligned}
& c_{1}=(\delta+\gamma \kappa) U W+V W(\gamma+\delta) \eta-U V(W+\gamma \kappa \eta / \delta), \\
& c_{2}=W[U+\eta V+(\gamma+\delta) W]
\end{aligned}
$$

In particular, the Routh-Hurwitz conditions [26] imply that $\mathcal{C}$ is stable only if $\delta U V W<0$. Since $\mathcal{C}$ is feasible if and only if $U, V, W$ have the same sign, a necessary condition for stability is $U, V<0$. Now, using $\zeta W=\vartheta U+\eta V$,

$$
\begin{gathered}
c_{1} c_{2}+\delta U V W^{3}=W[U+\eta V+(\gamma+\delta) W]\left\{U^{2} \vartheta(\delta+\gamma \kappa) / \zeta\right. \\
+V^{2}(\gamma+\delta) \eta^{2} / \zeta+\eta U V[(\gamma+\delta) \vartheta / \zeta \\
+(\delta+\gamma \kappa) / \zeta-\gamma \kappa / \delta]\} \\
-U V W^{2}(U+\eta V+\gamma W) .
\end{gathered}
$$

In particular, a sufficient condition for $c_{1} c_{2}+\delta U V W^{3}>0$ under $U, V, W<0$ and hence for $\mathcal{C}$ to be stable by the RouthHurwitz conditions is $\delta[\delta+\vartheta(\gamma+\delta)]+\gamma \kappa(\delta-\zeta)>0$. If $\delta>\zeta$, this holds true; if $\delta<\zeta$, it holds assuming the persister scalings (10), but $c_{1} c_{2}+\delta U V W^{3}<0$ and hence instability can occur if $\delta<\zeta$ and the persister scalings are not satisfied.

\section{b. Stability of coexistence if $\beta \neq 0$}

Finally, we discuss the stability of the two coexistence states $\mathcal{C}_{ \pm}=\left(b_{ \pm}, p_{ \pm}, a_{ \pm}\right)$, defined in Eqs. (E1) for $\beta \kappa \neq 0$. The Jacobian in Eq. (E6) has characteristic polynomial

$$
P_{ \pm}(\lambda)=\lambda^{3}+c_{2}^{ \pm} \lambda^{2}+c_{1}^{ \pm} \lambda+c_{0}^{ \pm},
$$

where, in particular and using $W=(\vartheta U+\eta V) / \zeta$,

$$
c_{0}^{ \pm}=a_{ \pm} b_{ \pm}\left[-\delta W+\beta \kappa\left(\eta a_{ \pm}-\vartheta b_{ \pm}\right)\right]=\mp a_{ \pm} b_{ \pm} \Delta,
$$

wherein $\Delta^{2}=X^{2}+4 \beta \delta \vartheta \kappa U=Y^{2}-4 \beta \delta \eta \kappa V$ and $\Delta>0$. Hence $c_{0}^{ \pm} \lessgtr 0$, and the Routh-Hurwitz conditions [26] imply in particular that $\mathcal{C}_{+}$is unstable. Further, on comparing to our earlier feasibility results, stable coexistence is only possible at $\mathcal{C}_{-}$and if $U, V<0$ or conditions (E4) are satisfied.

Moreover, $c_{2}^{-}=b_{-}+(\beta+\eta) a_{-}+\gamma+\delta>0$ if $\mathcal{C}_{-}$is feasible, and hence, by the Routh-Hurwitz conditions, if $\mathcal{C}_{-}$is feasible, it is stable if and only if $c_{1}^{-} c_{2}^{-}>c_{0}^{-}$. Although, as in Appendices $\mathrm{C}$ and $\mathrm{D}$, we have no complete characterization of the region of parameter space in which this condition holds, we can show that $\mathcal{C}_{-}$is stable in certain limits.

First, we notice that $\mathcal{C}_{-}$is stable for sufficiently large $\beta$. This follows from the expansion

$$
c_{1}^{-} c_{2}^{-}-c_{0}^{-}=\frac{\zeta^{3}}{\eta^{2}} \beta^{2}+O(\beta)
$$

obtained using Mathematica to assist with manipulating complicated algebraic expressions.

Next, $\Delta=|\beta \zeta \kappa-V \delta \eta / \zeta|+O(U)$ from its definition. If $V<0$, then $\beta \zeta^{2} \kappa>V \delta \eta$ is clearly true. This inequality also holds if $V>0$ and $\mathcal{C}_{-}$is feasible, from Eqs. (E4). Hence $\Delta=(\beta \zeta \kappa-V \delta \eta / \zeta)+O(U)$, and we find

$$
c_{1}^{-} c_{2}^{-}-c_{0}^{-}=\frac{\zeta}{\eta^{2}} u(u+\zeta \eta)+O(U)>0,
$$

where $u=\beta \zeta+(\gamma+\delta) \eta$. Hence $\mathcal{C}_{-}$is stable for small enough $|U|$ if it is feasible, i.e. if $U, V<0$ or conditions (E4) hold.

Moreover, as $\beta \rightarrow 0$ at $\kappa \neq 0, \mathcal{C}_{-} \rightarrow \mathcal{C}$, so, by continuity, $\mathcal{C}_{-}$is stable for small enough $\beta$, since $\mathcal{C}$ is, at least under the conditions discussed above and in particular if the persister scalings (10) are satisfied.

Finally, we discuss the stability of $\mathcal{C}_{-}$on, and hence by continuity near, the feasibility boundary $\beta=\beta_{*}$ defined in Eqs. (E4). On this boundary, $\Delta=0$ and hence $c_{0}^{-}=0$. Since $c_{2}^{-}>0$, stability is equivalent with $c_{1}^{-}>0$ there, as in Appendix C. Direct computation yields

$$
\begin{aligned}
(2 \beta \kappa)^{2} \eta \vartheta c_{1}^{-}= & \beta \kappa(\beta \zeta \kappa-\delta W)[\beta \zeta \kappa+\delta W+2 \eta(\delta+\gamma \kappa)] \\
& +(\beta \zeta \kappa+\delta W)[(\alpha \vartheta-\eta)(2 \beta \delta \vartheta-\beta \zeta \kappa+\delta W) \\
& +2 \beta \delta \kappa \eta \vartheta] . \quad \text { (E13a) }
\end{aligned}
$$

From Eqs. (E4), feasibility requires $V>0$, and so, from definition (E2a), $\vartheta>\zeta$. Moreover, we expect, from the persister scalings (10), that $\delta>\kappa$. Thus $2 \beta \delta \vartheta-\beta \zeta \kappa>\beta \zeta \kappa$. Since $\beta \zeta \kappa>\delta|W|$, Eq. (E13a) then shows that $c_{1}^{-}>0$ provided that $\alpha \vartheta>\eta$. Conversely, if $\alpha \vartheta<\eta$ or $\kappa<\delta$, then $c_{1}^{-}<0$ is possible, but we are not aware of simple conditions that ensure the presence or absence of instability in that case. However, after inserting the explicit expressions for $\beta=\beta_{*}$ and $W$ in terms of the other model parameters into Eq. (E13a) and expanding using Mathematica,

$$
c_{1}^{-}=\frac{64 \delta^{3} \eta^{3} \vartheta^{3}}{\zeta^{4}}+O\left(\zeta^{-3}\right)
$$

and hence $\mathcal{C}_{-}$is stable on the feasibility boundary $\beta=\beta_{*}$ for sufficiently small $\zeta$. We stress that this does not follow from Eq. (E12a), since the "sufficiently large" there depends on the other model parameters and, in particular, on $\zeta$. Again expressing $\beta=\beta_{*}$ and $W$ in terms of the other model parameters, we also find

$$
\begin{aligned}
c_{1}^{-} & =\frac{4 \delta \eta}{\vartheta}(\delta+\gamma \kappa)^{2} W^{2} & & \text { if } \zeta=\vartheta\left(1+\frac{\gamma \kappa}{\delta}\right)^{-1}, \\
& =\frac{4 \alpha \delta^{3} \vartheta}{\eta \kappa}(\alpha \vartheta-\eta+\eta \kappa) W^{2} & \text { if } \zeta=\frac{\eta}{\alpha} . & \text { (E13 })
\end{aligned}
$$

If $\eta / \alpha<\vartheta /(1+\gamma \kappa / \delta)<\vartheta$, then $\alpha \vartheta-\eta>0$. This therefore shows that $c_{1}^{-}>0$ at $\zeta=\min \{\eta / \alpha, \vartheta /(1+\gamma \kappa / \delta)\}$, and hence, on referring to Eq. (E5), that $\mathcal{C}_{-}$is stable at the "endpoint" of the feasibility boundary under discussion. For $\zeta=\eta / \alpha$, this result confirms a particular case of the conclusion that we drew from Eq. (E12b).

Combining these stability results yields the qualitative stability diagrams in Fig. 7, which we discuss in the main text. 


\section{Stability of the trivial steady states and permanence}

Finally, we analyze the stability of the trivial steady states to obtain conditions for permanent coexistence. As usual, $\mathcal{O}$ is always unstable, with the Jacobian of Eqs. (9) at this fixed point being

$$
\left(\begin{array}{ccc}
1-\gamma & \delta & 0 \\
\gamma & -\delta & 0 \\
0 & 0 & \zeta
\end{array}\right)
$$

Again omitting entries that do not affect the stability, the Jacobian at $\mathcal{A}$ is

$$
\left(\begin{array}{ccc}
-U / \eta-(\gamma+\beta \zeta / \eta) & \delta & 0 \\
\gamma+\beta \zeta / \eta & -\delta & 0 \\
& & -\zeta
\end{array}\right)
$$

The Jacobians at $\mathcal{O}$ and $\mathcal{A}$ are thus actually equal to the Jacobians of the corresponding steady states for model (7) given by Eqs. (C18a) and (C18b). Moreover, the Jacobian evaluated at $\mathcal{B}$ is

$$
\left(\begin{array}{ccc}
-\gamma-(1+\gamma \kappa / \delta)^{-1} & \delta-\kappa(1+\gamma \kappa / \delta)^{-1} & \\
\gamma & -\delta & -V(1+\gamma \kappa / \delta)^{-1}
\end{array}\right)
$$

On referring to the results in Appendix $\mathrm{C}, \mathcal{A}$ is stable if and only if $U>0$. Moreover, on computing the eigenvalues of the matrix in Eq. (E14c), we find that, again, $\mathcal{B}$ is stable if and only if $V>0$.

What is more, the eigenvectors of Jacobians in Eqs. (E14) are easily seen to have the same geometric properties as those of the corresponding Jacobians of model (7) analyzed in Appendix $\mathrm{C}$. We can therefore conclude, as we have done there, that the two species coexist permanently, irrespective of the initial conditions, if $\mathcal{A}$ and $\mathcal{B}$ are unstable, i.e. if $U, V<0$.

\section{APPENDIX F: STABILITY OF THE TRIVIAL STEADY STATES OF THE FULL MODEL (6) AND PERMANENT COEXISTENCE}

While we did not obtain any analytical results bearing on the stability (or indeed the feasibility) of the coexistence equilibria of Eqs. (6), more meaningful progress can be made as far as the stability of the trivial steady states is concerned. These results extend our results for the simplified models by proving that coexistence is permanent for model (6) whenever these trivial steady states are all unstable.

We begin by noting that the trivial steady state $\mathcal{O}=(0,0,0)$ of model (6) is always unstable. Next, Eqs. (6) have a trivial steady state $\mathcal{A}=(0,0, \zeta / \eta)$, which is feasible for all parameter values. The Jacobian of Eqs. (6) evaluated at $\mathcal{A}$ is

$$
\left(\begin{array}{ccc}
1-(\alpha+\beta) \zeta / \eta-\gamma & \delta & 0 \\
\gamma+\beta \zeta / \eta & -\tau-\xi \zeta / \eta & 0 \\
& & -\zeta
\end{array}\right)
$$

wherein, again, $\tau=\delta-\mu$ and entries left blank clearly do not affect stability; we shall assume that $\tau>0$, consistently with the persister scalings (10). This Jacobian has one eigenvalue $-\zeta<0$, which results from the trivial connection $\mathcal{O} \rightarrow \mathcal{A}$. Classical stability results [26] imply that $\mathcal{A}$ is stable if and only if the sub-Jacobian

$$
\left(\begin{array}{cc}
1-(\alpha+\beta) \zeta / \eta-\gamma & \delta \\
\gamma+\beta \zeta / \eta & -\tau-\xi \zeta / \eta
\end{array}\right)
$$

has $\operatorname{tr}<0$ and det $>0$. These conditions are at most quadratic in $\zeta$ and can be therefore be solved to show that

$$
\operatorname{tr}<0 \Longleftrightarrow \zeta>\zeta_{0}, \quad \operatorname{det}>0 \Longleftrightarrow \zeta_{-}<\zeta<\zeta_{+},
$$

where

$$
\begin{aligned}
& \zeta_{0}=\frac{(1-\gamma-\tau) \eta}{\alpha+\beta+\xi} \\
& \zeta_{ \pm}=\frac{\eta}{2(\alpha+\beta) \xi}\left(-z \pm \sqrt{z^{2}+4(\alpha+\beta) \xi(\tau+\gamma \mu)}\right)
\end{aligned}
$$

with $z=\alpha \tau-\beta \mu-(1-\gamma) \xi$. In particular, $\zeta_{-}<0$. Also, if $\zeta=\zeta_{0}$, then det $=-\left[1-\gamma-(\alpha+\beta) \zeta_{0} / \eta\right]^{2}-\delta\left(\gamma+\beta \zeta_{0} / \eta\right)<0$, so $\zeta_{-}<\zeta_{0}<\zeta_{+}$. Hence the necessary and sufficient condition for stability of $\mathcal{A}$ is $\max \left\{\zeta_{0}, 0\right\}<\zeta<\zeta_{+}$.

More importantly, even if the sub-Jacobian in Eq. (F1b) describes a saddle, its eigenvectors are

$$
\left(-\phi \pm \sqrt{\phi^{2}+4 \delta \eta(\beta \zeta+\gamma \eta)}, 2(\beta \zeta+\gamma \eta)\right)
$$

with $\phi=(\alpha+\beta) \zeta-(1+\tau-\gamma) \eta-\zeta \xi$, and so one of them is not feasible. This shows that the geometric properties of $\mathcal{A}$ match those of the corresponding trivial steady state of Eqs. (7) discussed in Appendix C.

If any more feasible trivial steady states exist, they are of the form $\mathcal{B}=(b, p, 0)$, where $b, p>0$ satisfy the simultaneous equations

$$
b(1-\gamma-b-\kappa p)+\delta p=p(-\tau-\varpi b-\varsigma p)+\gamma b=0 .
$$

The Jacobian of Eqs. (6) evaluated at $\mathcal{B}$ is, on simplification using Eqs. (F5),

$$
\left(\begin{array}{ccc}
-\delta q-b & \delta-\kappa b & \\
\gamma-\varpi p & -\gamma / q-\varsigma p & \\
0 & 0 & \zeta-\vartheta b-\iota p
\end{array}\right)
$$

where $q=p / b$ and, once again, entries left blank do not affect stability. This Jacobian has one eigenvalue $\zeta-\vartheta b-\iota p$, associated with perturbations out of the plane $A=0$, which may be of either sign. We note that the sub-Jacobian

$$
\left(\begin{array}{cc}
-\delta q-b & \delta-\kappa b \\
\gamma-\varpi p & -\gamma / q-\varsigma p
\end{array}\right)
$$

has trace and determinant

$$
\begin{aligned}
\operatorname{tr} & =-\delta q-b-\gamma / q-\varsigma p<0 \\
\operatorname{det} & =\delta p(\varsigma q+\varpi)+\gamma b(1 / q+\kappa)+(\varsigma-\kappa \varpi) p b>0,
\end{aligned}
$$

respectively. We have assumed, in the final line and consistently with the persister scalings (10), that $\varsigma>\kappa \varpi$. [It 
is because of the need for this additional assumption in this argument that we have provided separate proofs of our permanence result for the simplified models (7), (8), (9), which do not require such an additional assumption.] It follows that the remaining eigenvalues at $\mathcal{B}$ have negative real parts, and that the stability of $\mathcal{B}$ is determined by the sign of $\zeta-\vartheta b-\iota p$; determining this sign requires solving Eqs. (F5). For our pur- poses, it suffices to note that these results also show that the geometric properties of $\mathcal{B}$ match those of the corresponding steady state in Appendix C.

Combining these results with those in Appendix $\mathrm{C}$ thus shows that, assuming $\varsigma>\kappa \varpi$ and $\tau>0$, coexistence in the full model (6) is permanent if all trivial steady states are unstable.
[1] R. M. May, Will a large complex system be stable?, Nature (London) 238, 413 (1972).

[2] C. A. Serván, J. A. Capitán, J. Grilli, K. E. Morrison, and S. Allesina, Coexistence of many species in random ecosystems, Nat. Ecol. Evol. 2, 1237 (2018).

[3] S. Allesina and S. Tang, Stability criteria for complex ecosystems, Nature (London) 483, 205 (2012).

[4] A. Mougi and M. Kondoh, Diversity of interaction types and ecological community stability, Science 337, 349 (2012).

[5] K. Z. Coyte, J. Schluter, and K. R. Foster, The ecology of the microbiome: Networks, competition, and stability, Science 350, 663 (2015).

[6] J. Grilli, T. Rogers, and S. Allesina, Modularity and stability in ecological communities, Nat. Commun. 7, 12031 (2016).

[7] J. Grilli, G. Barabás, M. J. Michalska-Smith, and S. Allesina, Higher-order competitive interactions stabilize dynamics in competitive network models, Nature (London) 548, 210 (2017).

[8] S. Butler and J. P. O'Dwyer, Stability criteria for complex microbial communities, Nat. Commun. 9, 2970 (2018).

[9] A. Roberts, The stability of a feasible random ecosystem, Nature (London) 251, 607 (1974).

[10] J. Grilli, M. Adorisio, S. Suweis, G. Barabás, J. R. Banavar, S. Allesina, and A. Maritan, Feasibility and coexistence of large ecological communities, Nat. Commun. 8, 14389 (2017).

[11] T. Gibbs, J. Grilli, T. Rogers, and S. Allesina, Effect of population abundances on the stability of large random ecosystems, Phys. Rev. E 98, 022410 (2018).

[12] L. Stone, The feasibility and stability of large complex biological networks: a random matrix approach, Sci. Rep. 8, 8246 (2018).

[13] J. W. Barron and T. Galla, Dispersal-induced instability in complex ecosystems, Nat. Commun. 11, 6032 (2020).

[14] D. S. Maynard, C. A. Serván, J. A. Capitán, and S. Allesina, Phenotypic variability promotes diversity and stability in competitive communities, Ecol. Lett. 22, 1776 (2019).

[15] P. A. Haas, N. M. Oliveira, and R. E. Goldstein, Subpopulations and stability in microbial communities, Phys. Rev. Research 2, 022036(R) (2020).

[16] E. Maisonneuve and K. Gerdes, Molecular mechanisms underlying bacterial persisters, Cell 157, 539 (2014).

[17] A. Harms, E. Maisonneuve, and K. Gerdes, Mechanisms of bacterial persistence during stress and antibiotic exposure, Science 354, aaf4268 (2016).

[18] J. L. Radzikowski, H. Schramke, and M. Heinemann, Bacterial persistence from a system-level perspective, Curr. Opin. Biotechnol. 46, 98 (2017).

[19] E. Kussell and S. Leibler, Phenotypic diversity, population growth, and information in fluctuating environments, Science 309, 2075 (2005).

[20] D. I. Andersson and D. Hughes, Microbiological effects of sublethal levels of antibiotics, Nat. Rev. Microbiol. 12, 465 (2014).

[21] N. Q. Balaban, J. Merrin, R. Chait, L. Kowalik, and S. Leibler, Bacterial persistence as a phenotypic switch, Science 305, 1622 (2004).
[22] B. Kerr, M. A. Riley, M. W. Feldman, and B. J. M. Bohannan, Local dispersal promotes biodiversity in a real-life game of rock-paper-scissors, Nature (London) 418, 171 (2002).

[23] T. L. Czárán, R. F. Hoekstra, and L. Pagie, Chemical warfare between microbes promotes biodiversity, Proc. Natl. Acad. Sci. USA 99, 786 (2002).

[24] B. C. Kirkup and M. A. Riley, Antibiotic-mediated antagonism leads to a bacterial game of rock-paper-scissors in vivo, Nature (London) 428, 412 (2004).

[25] T. Reichenbach, M. Mobilia, and E. Frey, Mobility promotes and jeopardizes biodiversity in rock-paper-scissor games, Nature (London) 448, 1046 (2007).

[26] J. D. Murray, in Mathematical Biology, Vol. I (Springer, Berlin, Germany, 2002) 3rd ed., Chap. 3, pp. 79-118 and Appendices, pp. 501-511.

[27] We imply, in Eqs. (1) and throughout Sec. II and Appendix A, elementwise multiplication of vectors and rows or columns of matrices by writing the corresponding symbols next to each other, and reserve dots to denote matrix multiplication.

[28] Equations (1) and all subsequent models in this paper are deterministic: the rates of stochastic switching are thus not random variables, but deterministic parameters.

[29] E. D. Kelsic, J. Zhao, K. Vetsigian, and R. Kishony, Counteraction of antibiotic production and degradation stabilizes microbial communities, Nature (London) 521, 516 (2015).

[30] The confidence intervals computed for Figs. 2(a),(b), 3, 4 are Wilson intervals [see, e.g., L. D. Brown, T. T. Cai, and A. DasGupta, Interval estimation for a binomial proportion, Stat. Sci. 16, 101 (2001)]; those computed for Fig. 2(c) are derived from the Fieller-type statistic introduced by J.-m. Nam and W. C. Blackwelder, Analysis of the ratio of marginal probabilities in a matched-pair setting, Stat. Med. 21, 689 (2002).

[31] We will use "permanent coexistence" rather loosely to mean the alternative to extinction of one or more species, but note that different mathematical definitions, some of which go (rather unhelpfully in the biological context of this paper) by the name of "persistence" rather than "permanence", are associated with this concept in the dynamical systems literature: see, e.g., the definitions in Sec. 3 of G. Butler and P. Waltman, Persistence in dynamical systems, J. Differ. Equations 63, 255 (1986), and the related discussion in Sec. 2 of V. Hutson and G. Vickers, A criterion for permanent coexistence of species, with an application to a two-prey one-predator system, Math. Biosci. 63, 253 (1983).

[32] The name "bacteria", which we use to have a simple way of referring to phenotype $\mathrm{B}$, is of course not strictly biologically accurate, because the persisters (phenotype $\mathrm{P}$ ) and competitors (phenotype A) are, biologically, bacteria, too.

[33] The variables $B$ and $P$ in Eqs. (6) must be nondimensionalized using the same dimensional scalings lest the switching terms in Eqs. (6a) and (6b) become unbalanced. We could have chosen a different scaling for $A$ in Eqs. (6) from that of $B$ and $P$ to set e.g. $\alpha=1$, but equal scalings make comparing competition 
strengths easier. In the same spirit, we have reused the dimensional scalings from Eqs. (6) to nondimensionalize $B^{\prime}, A^{\prime}$ in the averaged model described by Eqs. (B1) to avoid having to unravel different dimensional scalings when writing down the consistency conditions expressed by Eqs. (B6).

[34] D. A. Cox, J. Little, and D. O'Shea, in Using Algebraic Geometry, Graduate Texts in Mathematics, Vol. 185 (Springer, Berlin, Germany, 2005) Chap. 2, pp. 26-76, 2nd ed.

[35] C.-K. Yap, in Fundamental Problems in Algorithmic Algebra (Oxford University Press, Oxford, England, 2000) Chap. 6 and Chap. 7, pp. 141-218.

[36] R. M. Stubbendieck, C. Vargas-Bautista, and P. D. Straight, Bacterial communities: Interactions to scale, Front. Microbiol. 7, 1234 (2016).

[37] T. S. Parker and L. O. Chua, in Practical Numerical Algorithms for Chaotic Systems (Springer, New York, NY, 1989) Chap. 3.2, Chap. 5.5, and App. B, pp. 61-66, 118-131, and 305-306.

[38] The approach of proving permanence by "chasing" possible limit sets in this way has also been used for general three-species Lotka-Volterra systems without phenotypic variation: see, e.g., H. I. Freedman and P. Waltman, Persistence in a model of three competitive populations, Math. Biosci. 73, 89 (1985) and G. J. Butler and P. Waltman, Persistence in three-dimensional LotkaVolterra systems, Math. Comput. Model. 10, 13 (1988); a more general approach for establishing permanence involves identifying an appropriate "averaged Lyapunov function" [see, e.g., J. Hofbauer and K. Sigmund, Permanence for replicator equations, in Dynamical Systems, Lecture Notes in Economics and Mathematical Systems, Vol. 287, edited by A. B. Kurzhanski and K. Sigmund (Springer, Berlin, Germany, 1987) pp. 70-92], but the corresponding general results (vide ibidem) cannot directly be applied to the systems with phenotypic switching considered here, because, for the two-species model (6) and its simplified versions (7), (8), (9), $\dot{B} / B$ and $\dot{P} / P$ diverge at $B=0$ and $P=0$, respectively.

[39] P. Glendinning, in Stability, Instability and Chaos: An Introduction to the Theory of Nonlinear Differential Equations (Cambridge University Press, Cambridge, UK, 1994) Chap. 5, pp. 102-144. 